Florida International University FIU Digital Commons

7-3-2013

\title{
Magnetic Nanoparticle-based Targeted Drug Delivery for Treatment of Neuro-AIDS and Drug Addiction
}

Vidya Sagar

vsaga001@fiu.edu

DOI: $10.25148 /$ etd.FI13080525

Follow this and additional works at: https://digitalcommons.fiu.edu/etd

Part of the Life Sciences Commons, and the Medicine and Health Sciences Commons

\section{Recommended Citation}

Sagar, Vidya, "Magnetic Nanoparticle-based Targeted Drug Delivery for Treatment of Neuro-AIDS and Drug Addiction" (2013). FIU Electronic Theses and Dissertations. 909.

https://digitalcommons.fiu.edu/etd/909 


\title{
FLORIDA INTERNATIONAL UNIVERSITY
}

Miami, Florida

\section{MAGNETIC NANOPARTICLE-BASED TARGETED DRUG DELIVERY FOR TREATMENT OF NEURO-AIDS AND DRUG ADDICTION}

\author{
A dissertation submitted in partial fulfillment of the \\ requirements for the degree of \\ DOCTOR OF PHILOSOPHY \\ in \\ BIOLOGY \\ by \\ Vidya Sagar
}

2013 
To: Dean Kenneth G. Furton

College of Arts and Sciences

This dissertation, written by Vidya Sagar, and entitled Magnetic Nanoparticlebased Targeted Drug Delivery for Treatment of NeuroAIDS and Drug Addiction, having been approved in respect to style and intellectual content, is referred to you for judgment.

We have read this dissertation and recommend that it be approved.

\section{Lidia Kos}

Alejandro Barbieri

Ravi Pottathil

Sakhrat Khizroev

Madhavan Nair, Major Professor

Date of Defense: July 03, 2013

This dissertation of Vidya Sagar is approved.

$\begin{array}{r}\text { Dean Kenneth G. Furton } \\ \text { College of arts and Sciences } \\ \hline \begin{array}{r}\text { Dean Lakshmi N. Reddi } \\ \text { University Graduate School }\end{array}\end{array}$

Florida International University, 2013 


\section{DEDICATION}

I dedicate this dissertation to flood victims of Uttarakhand, India. Thousands innocent people lost their lives as Mother Nature striked. May the lost souls rest in peace! 


\section{ACKNOWLEDGEMENTS}

I soulfully acknowledge my gratitude towards people who raised their helping hands in a number of ways during my academic life and especially during this study. While few names are being mentioned and shall be read between the lines, I owe deep seated gratitude to all missing names.

At first, I express my reverence to "God Almighty" for affording me enormous strength to overcome the adversities in my life. I fell short of words to express my wholehearted gratitude to my parents and siblings whose blessings, selfless and unfeigned love and empathy, and sky high inspirations became the guiding force of my life and propelled me towards brighter horizon of happiness.

It is exquisitely a jubilating occasion and unique opportunity to express my deepest sense of gratitude and indebtedness to my mentor, Dr. Madhavan Nair, to whom I owe my deepest respects. With profound regards, I extend my gratitude to committee members, Dr. Lidia Kos, Dr. Manuel Barbieri, Dr. Ravi Pottathil, and Dr. Sakhrat Khizroev for supporting this research and constant encouragement and great human gesture during preparation of the dissertation. I shall be grateful to the Florida International University, especially to the department of biology (college of arts and sciences) and department of immunology (college of medicine) for their substantial teaching and research assistance/ facilities respectively. I owe my obligations to all the faculty and staff members of these departments for their cordial co-operation and encouragement given during my study. I also sincerely acknowledge MBRS-RISE Biomedical Research Initiative, for granting student summer research award for this work.

Lastly, it is my proud privilege and immense pleasure to acknowledge my well-wishers for luminous inspiration and efforts in planning and execution of the work during the entire course of my study and incessant blessings and devotion. 


\section{ABSTRACT OF THE DISSERTATION \\ MAGNETIC NANOPARTICLE-BASED TARGETED DRUG DELIVERY FOR TREATMENT OF NEURO-AIDS AND DRUG ADDICTION}

by

Vidya Sagar

Florida International University, 2013

Miami, Florida

Professor Madhavan Nair, Major Professor

Brain is one of the safe sanctuaries for HIV and, in turn, continuously supplies active viruses to the periphery. Additionally, HIV infection in brain results in several mild-to-severe neuro-immunological complications termed neuroAIDS. One-tenth of HIV-infected population is addicted to recreational drugs such as opiates, alcohol, nicotine, marijuana, etc. which share common target-areas in the brain with HIV. Interestingly, intensity of neuropathogenesis is remarkably enhanced due to exposure of recreational drugs during HIV infection. Current treatments to alleviate either the individual or synergistic effects of abusive drugs and HIV on neuronal modulations are less effective at CNS level, basically due to impermeability of therapeutic molecules across blood-brain barrier (BBB).

Despite exciting advancement of nanotechnology in drug delivery, existing nanovehicles such as dendrimers, polymers, micelles, etc. suffer from the lack of adequate BBB penetrability before the drugs are engulfed by the reticuloendothelial system cells as well as the uncertainty that if and when the nanocarrier reaches the brain. Therefore, in order to develop a fast, target- 
specific, safe, and effective approach for brain delivery of anti-addiction, anti-viral and neuroprotective drugs, we exploited the potential of magnetic nanoparticles (MNPs) which, in recent years, has attracted significant importance in biomedical applications. We hypothesize that under the influence of external (non-invasive) magnetic force, MNPs can deliver these drugs across BBB in most effective manner. Accordingly, in this dissertation, I delineated the pharmacokinetics and dynamics of MNPs bound anti-opioid, anti-HIV and neuroprotective drugs for delivery in brain. I have developed a liposome-based novel magnetized nanovehicle which, under the influence of external magnetic forces, can transmigrate and effectively deliver drugs across BBB without compromising its integrity. It is expected that the developed nanoformulations may be of high therapeutic significance for neuroAIDS and for drug addiction as well. 


\section{TABLE OF CONTENTS}

CHAPTER

PAGE

1.0 Introduction:

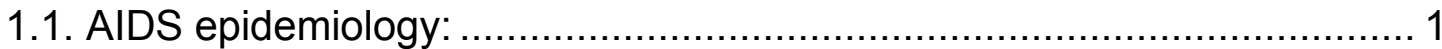

1.2.HIV, drugs of abuse and CNS impairment: …............................... 3

1.2.1.Neurobiology of morphine addiction and effect on HIV

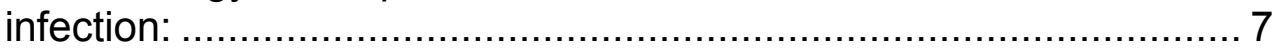

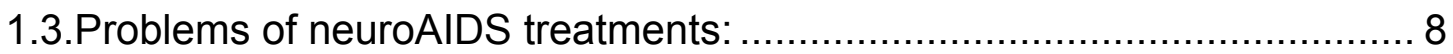

1.3.1. Limitations of current treatments:............................................... 8

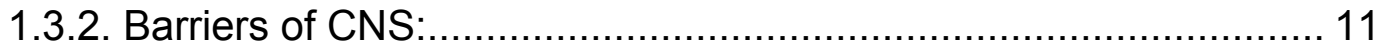

1.4.Advantages of nano-scale technology in drug-delivery ........................ 17

1.5. Nanomedicines for neuroAIDS treatment ........................................ 21

1.6. Functional nanovehicles for prevention and treatment of neuroAIDS: $\ldots . .22$

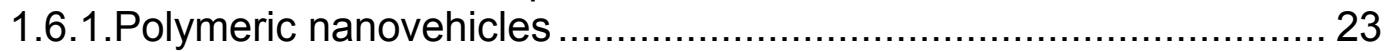

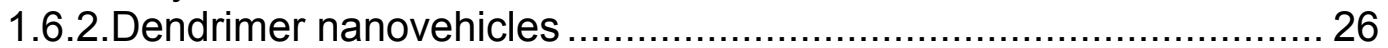

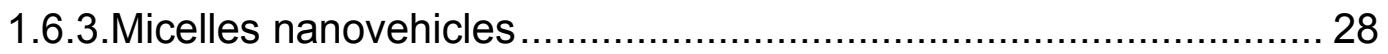

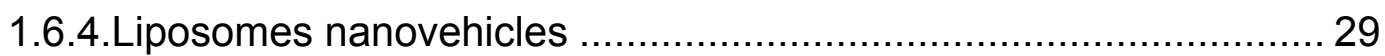

1.6.5.Solid lipid nanoparticles (SLN) based nanovehicles ..................... 31

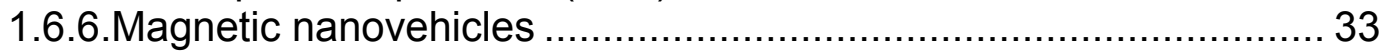

1.6.7.Cell-based nanovehicles..................................................... 36

1.6.8. Other promising nanovehicles for ARV drug delivery across BBB.. 40

1.7. Nanovehicles mediated delivery of anti-abuse drugs for treatment of

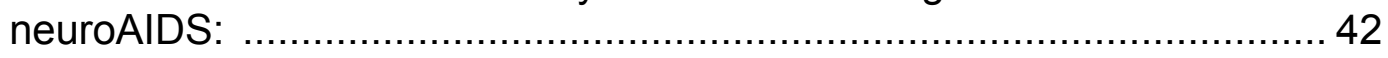

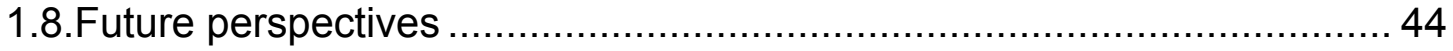

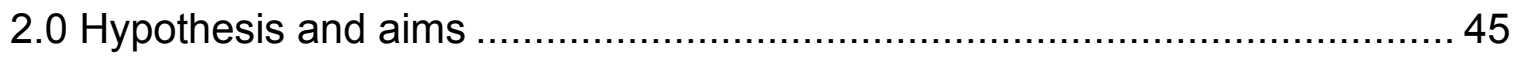

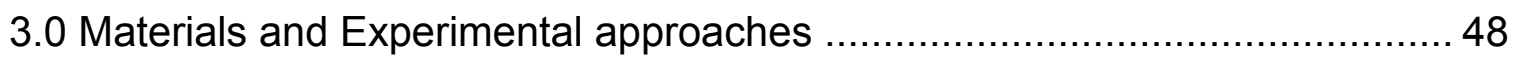

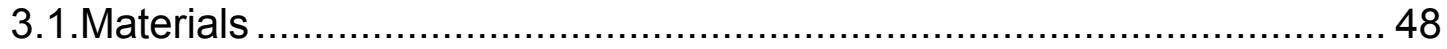

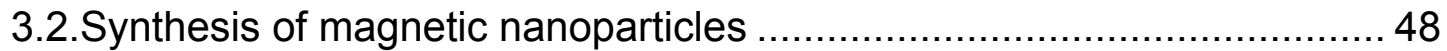

3.3. Characterization of MNPs ......................................................... 49

3.3.1.X-ray diffraction (XRD) and transmission electron microscopy

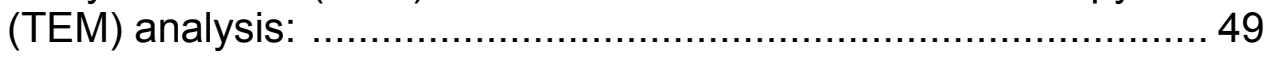

3.3.2. Particle size and zeta potential ……...................................... 50

3.3.3. Superparamagnetism measurement ..................................... 50

3.4. Binding of drugs onto the magnetic nanoparticles ................................ 50

3.4.1.CTOP binding to magnetic nanoparticles ................................... 50

3.4.1.1. High-performance liquid chromatography/Photo diode array (HPLC/PDA): ................................................... 50

3.4.1.2. Fourier transform infrared spectroscopy (FTIR) ................ 51

3.4.1.3.Fluorescent tagging of CTOP for binding validation........... 51

3.4.2.BDNF binding with magnetic nanoparticles ............................... 52 
3.4.2.1.BDNF enzyme linked immunosorbent assay (ELISA)............. 52

3.4.2.2. Fluorescent tagging of BDNF for binding validation................ 53

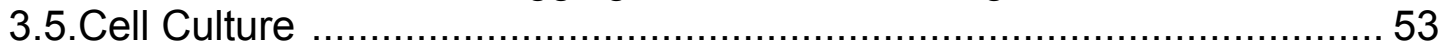

3.5.1.Preparation of Peripheral blood mononuclear cells (PBMC)...........5 53

3.5.2.Peripheral blood mononuclear cells (PBMC) culture ..................... 54

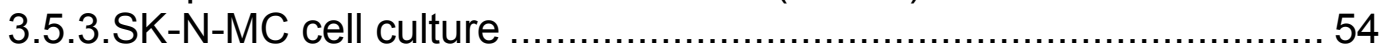

3.5.4.Primary human astrocytes (HA) culture ....................................... 54

3.5.5. Human brain endothelial cell (HMBVEC) culture ............................ 55

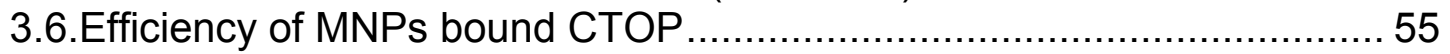

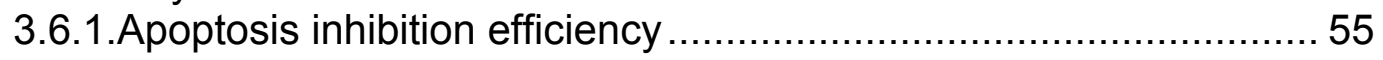

3.6.2. Characterization of neuro-spinal architecture .............................. 56

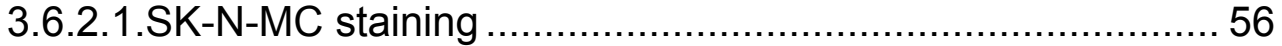

3.6.2.2. HIV co-infection of SK-N-MC with morphine treatments ..... 57

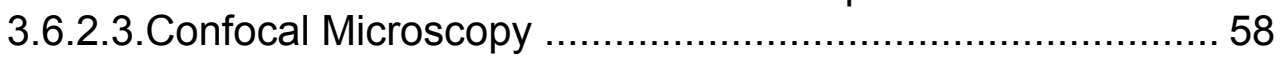

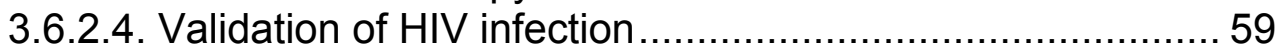

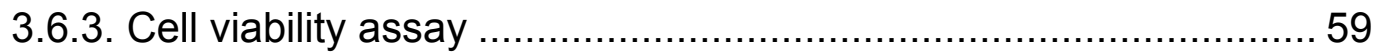

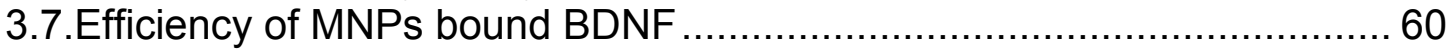

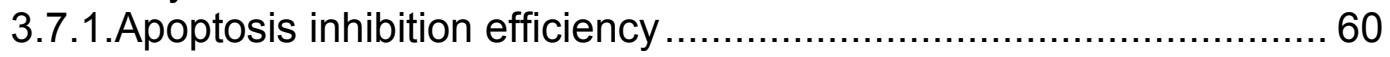

3.7.2. Quantification of cAMP response element-binding protein

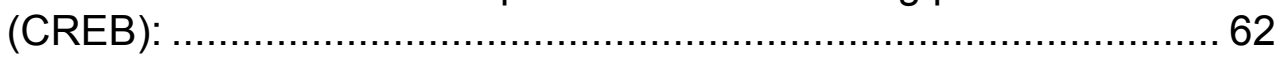





3.7.2.3. Quantitative polymerase chain reaction (qPCR): ................ 63

3.7.2.4.Calculation of transcript accumulation index (TAI) or relative expression

3.7.3. Characterization of neuro-spinal architecture: SK-N-MC

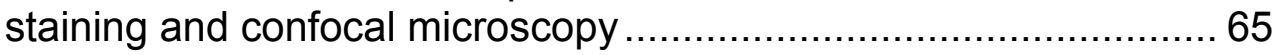

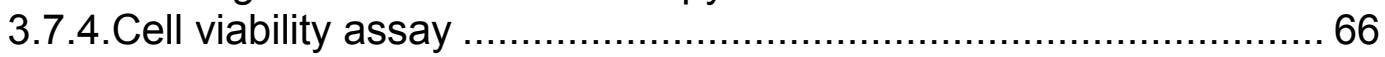

3.8. Formulation of liposomes-based magnetic nanocarriers ....................... 67

3.8.1.Synthesis of ultrasmall magnetic nanoparticles ............................ 67

3.8.2.Formulation of PEGylated magneto-liposome and transferrin

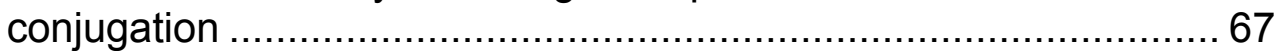

3.8.2.1.Validation of Transferrin conjugation ............................... 68

3.8.3.Determination of encapsulation efficiency (EE) .............................68

3.8.3.1. Quantitation of encapsulated MNPs in liposome .............69 69

3.8.4.Determination of colloidal- and fluorescent-integrity of



3.9. In vitro blood-brain barrier (BBB) and nanocarrier transmigration ........... 70

3.9.1.Preparation of in vitro BBB model.............................................. 70

3.9.2. Transmigration of fluorescent magneto-liposomes across in

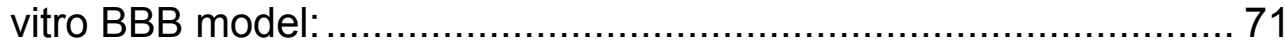

3.9.3. Transmigration and efficiency of BDNF .................................... 72

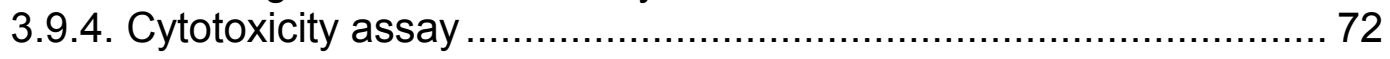




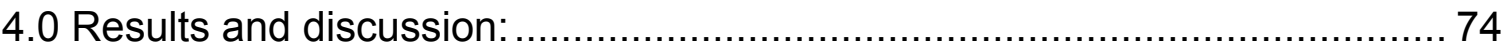

4.1.Characterization of magnetic nanoparticles....................................... 75

4.2. Effect of $\mathrm{pH}$ on surface charge distribution of MNPs ........................... 76

4.3.CTOP adsorption on MNPs surface .............................................. 77

4.4. Time kinetics and binding isotherm of CTOP to MNPs: ........................ 81

4.5.Functional efficiency of MNPs bound CTOP........................................ 82

4.5.1. Inhibition of morphine-induced peripheral pathogenesis............... 82

4.5.2. Inhibition of morphine-induced neuronal pathogenesis.................. 84

4.5.3. Inhibition of morphine-induced neuronal pathogenesis during

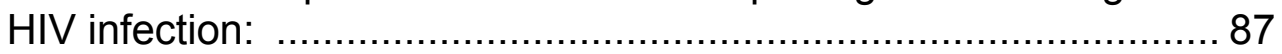

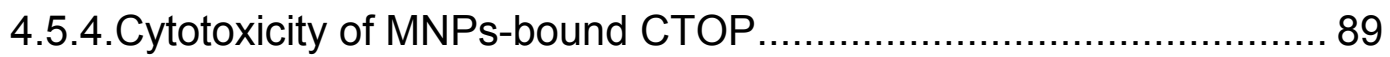

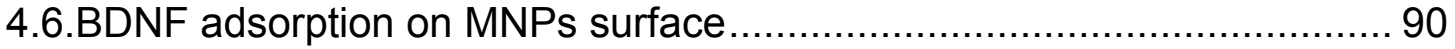

4.6.1.Time kinetics and binding isotherm of BDNF to magnetic nanoparticles: ..................................................................... 91

4.7.Apoptosis inhibition efficiency of MNPs bound BDNF in leukocytes ........ 92

4.8. Efficacy of MNPs bound BDNF in Astrocytes: ..................................... 94

4.8.1. Modulation of CREB expression in Astrocytes ........................... 94

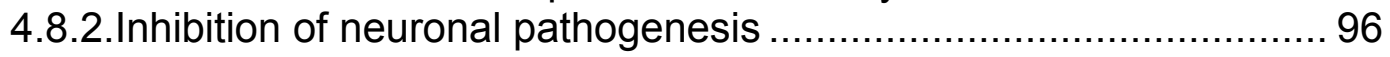

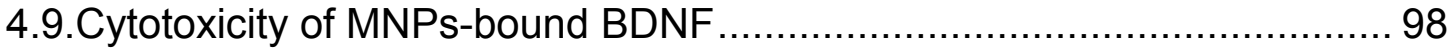

4.10.Characterization of MNPs-based liposomal nanocarriers .................... 99

4.10.1.Characterization of ultrasmall magnetic nanoparticles....................... 99

4.10.2. Characterization of $\mathrm{ML}$ nanocarriers......................................... 103

4.10.3.Physiological sustainability of $M L$ nanocarriers............................ 108

4.11. Transmigration of $\mathrm{ML}$ nanocarriers across BBB …............................. 110

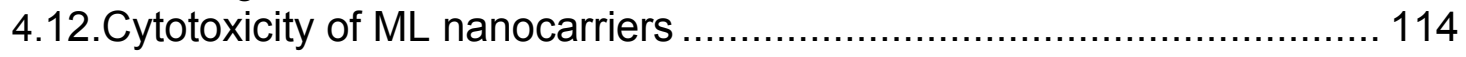

4.13.BBB transmigration and efficiency of BDNF nanoformulations ............ 115

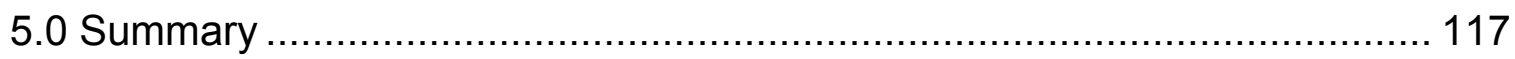

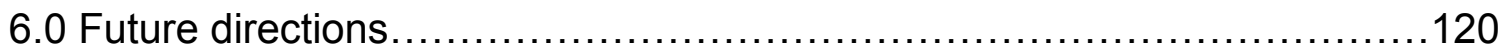

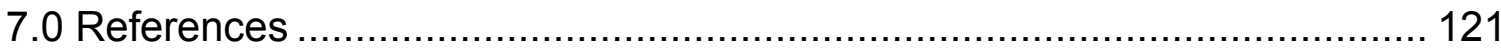

VITA … 


\section{LIST OF FIGURES}

FIGURE

PAGE

Figure 1: $\quad$ Simplified overview of the common pathways involved in HIV and Recreational drugs induced neuropathogenesis.

Figure 2: Nanoparticles used for the delivery of drugs across BBB 24

Figure 3: Magnetic Nanoparticles based nanovehicles: Magnetoliposome for drug delivery across BBB. 35

Figure 4: $\quad$ Cell-based drug delivery: Monocytes/Macrophages loaded with liposomal nanocarriers.

Figure 5: Schematic of the experimental design applied for apoptosis inhibition assay with MNPs-CTOP nanoformulations .................... 56

Figure 6: $\quad$ Schematic of the experimental design applied for efficiency determination of MNPs-CTOP nanoformulations on morphine and HIV co-infection induced neuropathogenesis. 57

Figure 7: Schematic of a typical dendrite segment and Spine density measurement 58

Figure 8: $\quad$ Schematic of the experimental design applied for cell-viability assay with MNPs-CTOP nanoformulations

Figure 9: Schematic of the experimental design applied for determination of optimum BDNF concentration required for inhibition of morphineinduced apoptosis in PBMCs 61

Figure 10: Schematic of the experimental design applied for apoptosis inhibition assay with MNPs-CTOP nanoformulations 61

Figure 11: Schematic of the experimental design applied for cell-viability assay with MNPs-BDNF nanoformulations 66

Figure 12: Transmission electron micrograph and Size distribution of $\mathrm{Fe}_{3} \mathrm{O}_{4}$ magnetic nanoparticles

Figure 13A: Zeta potential (surface charge) of MNPs at different $\mathrm{pH}$ 77

Figure 13B: Schematic illustration of proposed electrostatic interaction between MNPs and different drugs at $\mathrm{pH} 7.4$. 
Figure 14: FTIR spectra of transmittance

Figure 15: Fluorescent-based CTOP binding verification ............................. 80

Figure 16: Time kinetics and Binding isotherm of CTOP onto MNPs ..........82

Figure 17: Flow-cytometry to evaluate the efficacy of MNPs bound CTOP on morphine induced apoptosis in PBMCs .................................... 83

Figure 18: Confocal microscopy to evaluate the efficacy of MNPs bound CTOP on morphine induced neuropathogenesis

Figure 19: Spinal density (No. of spines/ $\mu \mathrm{m}$ dendritic length) of SK-N-MC showing morphine induced spinal degeneration and effect of Free and MNP bound CTOP on prevention of this degradation 86

Figure 20: Confocal microscopy to evaluate the efficacy of MNPs bound CTOP on morphine and HIV co-infection induced neuropathogenesis 88

Figure 21: Spinal density (No. of spines/ $\mu$ m dendritic length) of SK-N-MC showing morphine and HIV co-infection induced spinal degeneration and effect of MNP bound CTOP on prevention of this degradation. 88

Figure 22: Percent cell viability of PBMCS and SK-N-MC cells 48 hrs posttreatment with MNPs and MNP-CTOP nanoformulations.

Figure 23: Fluorescent-based BDNF binding verification: Immobilization of red fluorescent dye tagged BDNF on MNPs emits fluorescence while MNPs without BDNF shows no fluorescence. 90

Figure 24: Binding isotherm of BDNF on MNPs:

Figure 25: Flow-cytometry to evaluate the efficacy of MNPs bound BDNF on morphine induced apoptosis in PBMCs

Figure 26: Trans-accumulation index (TAI) showing CREB expression 95

Figure 27: Efficacy of MNPs bound BDNF on morphine induced neuropathogenesis

Figure 28: Percent cell viability of PBMCS $48 \mathrm{hrs}$ post-treatment with MNPs and MNP-BDNF nanoformulations. 
Figure 29: Characterization of ultrasmall magnetite nanoparticles 101

Figure 30A: Magnetic hysteresis loop of MNPs

Figure 30B: Surface charge of MNPs varies according to $\mathrm{pH}$ values of suspension solution 102

Figure 31: Characterization of magnetic-liposomes (MLs) 3 105

Figure 32: Characterization of transferrin conjugation to MLs 107

Figure 33: Characterization of MLs sustainability 110

Figure 34: Relative and percentile transmigration of ML nanocarriers in the presence and absence of external magnetic force across in vitro BBB at different time points

Figure 35: The $\mathrm{Fe}_{3} \mathrm{O}_{4}$ content in lower chamber post-transmigration 114

Figure 36: Percent cell viability of primary endothelial cells and astrocyte cells 24 and 48 hrs post-treatment with different concentration of ML nanocarriers

Figure 37: Percentage transmigration and TEER of free and MNP-BDNF nanoformulations

Figure 38: Trans-accumulation index (TAI) showing effect of Free BDNF and MNPs-BDNF nanoformulations on CREB expression in Astrocytes across BBB 116

Figure 39: Proposed shcematic of magnetic nanoparticles based drugs delivery across BBB 119

Figure 40: Shcematic of future work 120 


\section{LIST OF ABBREVIATIONS AND ACRONYMS}

- ABC: ATP-binding cassette

- AM: Astrocyte medium

- ARV: Anti-retro viral

- AZTTP: 3'-azido-3' -deoxythymidine-5' -triphosphate

- BBB: Blood-brain barrier

- BCRP: Breast cancer resistance protein

- BCSFB: Blood-cerebrospinal fluid barrier

- BDNF: brain derived neurotropic factor

- BMECs: Brain microvascular endothelial cells

- $\quad$ cDNA: Complimentary DNA

- CFPE: 1,2-distearoyl-sn-glycero-3-phosphoethnolamine-Ncarboxylfluorescein

- CNS: Central nervous system

- CNTs: Concentrative nucleoside transporters

- $\quad$ CREB: cAMP response element-binding protein

- CSF: Cerebrospinal fluid

- CSFB: Cerebrospinal fluid-brain barrier

- CTOP: D-Pen-Cys-Tyr-DTrp-Orn-Thr-Pen-Thr-NH2

- DLS: Dynamic laser scattering

- DNA: Deoxyribose nucleic acid

- ECM: Endothelial cell medium

- EE: Encapsulation efficiency 
- ELISA: Enzyme linked immunosorbent assay

- EMF: Electromagnetic force

- ENTs: Equilibriative nucleoside transporters

- Fl: Fusion Inhibitors

- FTIR: Fourier transform infrared spectroscopy

- HA: Human astrocytes

- HAART: Highly Active Antiretroviral Therapy

- HAD: HIV associated dementia

- HBMVE: Human brain endothelial cell

- HIV: Human immunodeficiency virus

- HIVE: HIV-1 encephalitis

- HPLC: High-performance liquid chromatography

- IL: Interleukin

- Inl: Integrase Inhibitors

- kDa: Kilo Dalton

- LNC: Lipid nanocapsules

- MDM: Monocyte-derived macrophages

- ML: Magneto-liposomes

- MMSPM: Methylmethacrylate-sulfopropylmethacrylate

- MNP: Magnetic nanoparticles

- $\mathrm{mPEG}_{2000}$-DSPE : 1,2-distearoyl-sn-glycero-3-phosphoethnol- amine-N[methyoxy(polyethylene glycol)-2000

- MRI: Magnetic resonance imaging 
- mRNA: Messenger RNA

- MRPs: Multi-drug resistance-associated proteins

- MTS: [3-(4, 5-dimethyl thiazol-2-yl)-5-(3-carboxy-methoxyphenyl)-2-(4sulfophenyl)-2H-tetrazolium, inner salt

- $\mathrm{mV}$ : Millivolt

- NNRTI: Non- Nucleoside Reverse Transcriptase Inhibitors

- NRTI: Nucleoside Reverse Transcriptase Inhibitors

- NtRTI: Nucleotide Reverse Transcriptase Inhibitors

- OATPs: Organic anions-transporting polypeptide

- OATs: Organic anion transporters

- OCTs: Organic cation transporter

- PBCA: Poly (butyl cyanoacrylate)

- PBMC: Peripheral blood mononuclear cells

- PCR: Polymerase chain reaction

- PDA: Photo diode array

- PEG: Poly-Ethyl-Glycol

- P-gp: P-glycoprotein

- PI: Protease Inhibitors

- PLA: Polylactide

- PLGA: Poly (lactide-co-glycolide

- qPCR: Quantitative polymerase chain reaction

- RNA: Ribose nucleic acid

- SDS: Sodium dodecyl sulphates 
- SLC: Solute-carrier:

- SLN: Solid lipid nanoparticles

- TAl: Transcript accumulation index

- TE : Tris-EDTA

- TEER: Trans-endothelial electrical resistance

- TEM: Transmission electron microscope

- XRD: X-ray diffraction 


\section{Introduction}

\subsection{AIDS epidemiology:}

Acquired immune deficiency syndrome (AIDS) is one of the most sought infectious diseases of the modern world (Castro-Nallar et al., 2012). It ranks among the top four cause of death throughout the globe - particularly in Africa and women of reproductive age group it is the number one cause of death (MMWR, 2001; UNAIDS, 2012 ${ }_{\mathrm{a}}$ ). Since it was discovered in the early 1980s that HIV is the causative agent of AIDS, about 65 million people throughout the world have been estimated to be infected (ILO, 2012). The Sub-Saharan African region remains the epicenter of this infection; however, certain other parts of the globe such as the Caribbean Island, America, Eastern Europe and Central Asia also fall in high alarm zones where HIV prevalence in adults are equal to or more than 0.5\% (UNAIDS, 2010). Almost 34.2 million people are currently living with this global pandemic where women account for half of the population (UNESCO, 2012; UNAIDS, 2012a). People from all age group including children and young adolescent are affected by this pandemic. While Children (age 0-15) alone constituted $10 \%$ of total people living with HIV in the year 2010 (UNAIDS, 2010), $42 \%$ of all new adult (15+ age) infections were found in the young aged 15-24 (UNAIDS, 2012b).

Human immunodeficiency virus (HIV) is transmitted mainly through sexual contact, contaminated injection equipment, and/or mother-to-child transmission. Sexual contact remains the primary source of transmissions and accounts for 
almost $80 \%$ of the HIV infections throughout the globe (Skar et al., 2011). Mother-to-child transmission resulted in the birth of approximately 370, $000 \mathrm{HIV}$ positive children in 2009; nevertheless, this is a declining trend as compare with 2001 when nearly 500, 000 cases were estimated (UNAIDS, 2010). The transmission of HIV as a result of contaminated injection continue to be the matter of concern. Predominantly among intravenous drug abusers, sharing of injection equipment is a common practice and thus, they are extremely vulnerable to explosive outbreaks of HIV infections as have been seen in many regions like Finland, Sweden, Thailand, and former Soviet Union. Globally, almost $10 \%$ of HIV infections are related to injection drug use which accounts for approximately one fifth of all injection drug users (UNAIDS/PCB, 2009; Skar et al., 2011). This proportion is more alarming in the United States, and Eastern Europe and Central Asia where, respectively, one third and one fourth of injection drug users are HIV-infected (UNAIDS, 2010; UNODC, 2012). Similarly, higher proportions of HIV infected people among injection drug users have been estimated in places like Nairobi (36\%), Zanzibar (27\%), Myanmar (38\%), Thailand ( $30 \%-50 \%)$, Vietnam $\quad(32 \%-58 \%)$, Indonesia $(50 \%)$, Russian Federation (37\%) and, Ukraine (39\%-50\%-Kryvyi Rih-88\%)( UNAIDS, 2010).

Implementation of antiretroviral therapies (ART) has come up as respite for AIDS patients. Nearly $21 \%$ decline in the AIDS-related death rate has been reported between the years 1997-2010 (The World Bank, 2012; UNAIDS, 2012). Nonetheless, HIVIAIDS still remains unstoppable and incurable. Alarmingly, 
almost 7000 new individuals were infected every day throughout the globe in 2010 (Douce et al., 2012) and nearly 9 million people living with HIV lack access of any of treatment (WHO, 2012). Additionally, quite unrealistically, uninterrupted treatment for several years has been theorized for complete viral eradication with existing ART (Perelson et al., 1997; Chun et al., 2007). Also, their therapeutic values have been shown to of small benefit for associated complications such as illness of central nervous system (CNS) (Vivithanaporn et al., 2011). Most importantly, limited or zero reachability of most antiretroviral drugs to viral tissue sanctuaries such as brain hampers the viral clearance and it is believed as the main reason of resurgence of HIV from below detection level to outbreak on interruption of the treatment (Varatharajan and Thomas., 2009; Douce et al., 2012). These, in association with epidemiological data, indicate a less promising trend towards faster eradication of HIV infections. Thus, significant improvements over the current therapies of HIVIAIDS are needed immediately.

\subsection{HIV, drugs of abuse and CNS impairment:}

Nervous system alterations as a result of direct or indirect effect of HIV infection, collectively known as neuroAIDS, are always associated with AIDS patients. At least $10 \%$ of diagnosed cases are accompanied by some kind of neurological illness (Almeida and Ellis, 2006) and further during the disease progression approximately $50 \%$ cases demonstrate neuropathological signs or symptoms (McArthur et al., 2005). In the same line, mild to severe neurological alterations are seen in at least $80 \%$ autopsies of AIDS patients (Almeida and 
Ellis, 2006). Many CNS diseases such as viral and chronic meningitis, HIVassociated neurocognitive disorders (HAND), vacuolar myelopathy, peripheral neuropathies, etc. have been coupled to the neurovirulent effects of HIV (Letendre et al., 2009; Singer et al., 2010).

Previously it was believed that penetration of HIV into CNS occurs only during the final stage of infection when higher load of virus is found in the peripheral blood. Now it is putatively believed that virus may enter the CNS from the onset of infection (Kramer-Hammerle et al., 2005). Towards this end, presence of HIV-particles, -proteins, and -DNA in the CNS along with the intrathecal production of anti-HIV antibodies have been seen during the initial infection (Ho et al., 1985; Resnick et al., 1985; An SF and Scaravilli, 1997, Rolfs and Schumacher, 1990; Davis et al., 1992; 1996; Gray et al., 1996; Almeida and Ellis, 2006). HIV may enter into CNS either directly or as "Trojan passenger" via trafficking of infected monocytes, macrophages, and/or T-cells across the tightly junctioned brain microvascular endothelial cells (BMECs) of blood-brain barrier (BBB) (Albright et al., 2003; Ghafouri et al., 2006). Initial infection of HIV in the CNS triggers production of proteins that alter the BBB integrity (e.g. matrix metalloproteinase) (Sporer et al., 1998; Conant et al., 1999) and influence leukocytes transmigration across this barrier (e.g., monocyte chemotactic protein-1) (Boven et al., 2000). These intensify the HIV infection resulting in degradation of BBB and CNS injury. Numbers of viral proteins have been shown to induce the HIV neurotoxicity and associated pathology. Particularly, HIV protein gp120 and Tat have been extensively studied. Both, gp120 and Tat can 
breach BBB independent of viral penetration and can be toxic across multiple species and cell lines. Several neuropathological features are noticed due to treatment of these proteins (Ghafouri et al., 2006; Ferris et al., 2008).

As discussed earlier, spread of HIV infection and drug abuse are significantly interlinked. Along the same line, drug abuse can alter the neuroplasticity and damage the CNS analogous to that happens during the HIV infections (e.g., loss of dopaminergic neurons) (Nath et al., 2002; Burdo et al., 2006). Most importantly, many illicit drugs have been shown to promote susceptibility/progression of HIV infections and associated neuropathogenesis. This stimulation of neuropathogenesis in drug-addicted AIDS patients can be attributed to the concerted effect of HIV (or its protein) and drugs of abuse on neurotoxicity. All sorts of abusive drugs such as psychomotor simulants (Amphetamines), opiates (cocaine, morphine), alcohol, nicotine, marijuana, etc. have been shown to cause concerted effect on the HIV-associated neuropathogenesis in one or other way (Hauser et al., 2007; Ferris et al., 2008; Rogers, 2011). For examples, the immunomodulatory actions of opioids induce the expression of $\mu$ and other chemokine receptors in monocytic cells resulting in increased HIV susceptibility and stimulation of HIV expression (Peterson et al., 1993; 1999; Rogers et al., 2000). Also, opiates enhance the production of proinflammatory factors like MCP-1, RANTES, IL-6, ROS, etc. in the brain cells such as neurons, astroglia and microglia (Peterson et al., 1998; El-Hage et al., 2005). These exacerbate the preexisting inflammation of neurons as a consequence of HIV infections. Additionally, alteration in endogenous opioids 
level cause disruption of dopaminergic functions which affect the neuroimmunological ability of nervous system to respond against HIV (Hauser et al., 2005; Reddy et al., 2012). Psychostimulants such as methamphetamine and cocaine have also been shown to disrupt the dopamine level resulting in oxidative damage of neurons (Cubells et al., 1994; Brown and Yamamoto, 2003; Riddle et al., 2006). Likewise, alcohol exposure alters the BBB permeability which leads to increased HIV entry and ROS level in the brain via influx of macrophages (Haorah et al., 2004; 2005; Persidsky et al., 2006). Thus, a concerted effect of drugs of abuse and HIV infection result in sever neurobiological alterations.

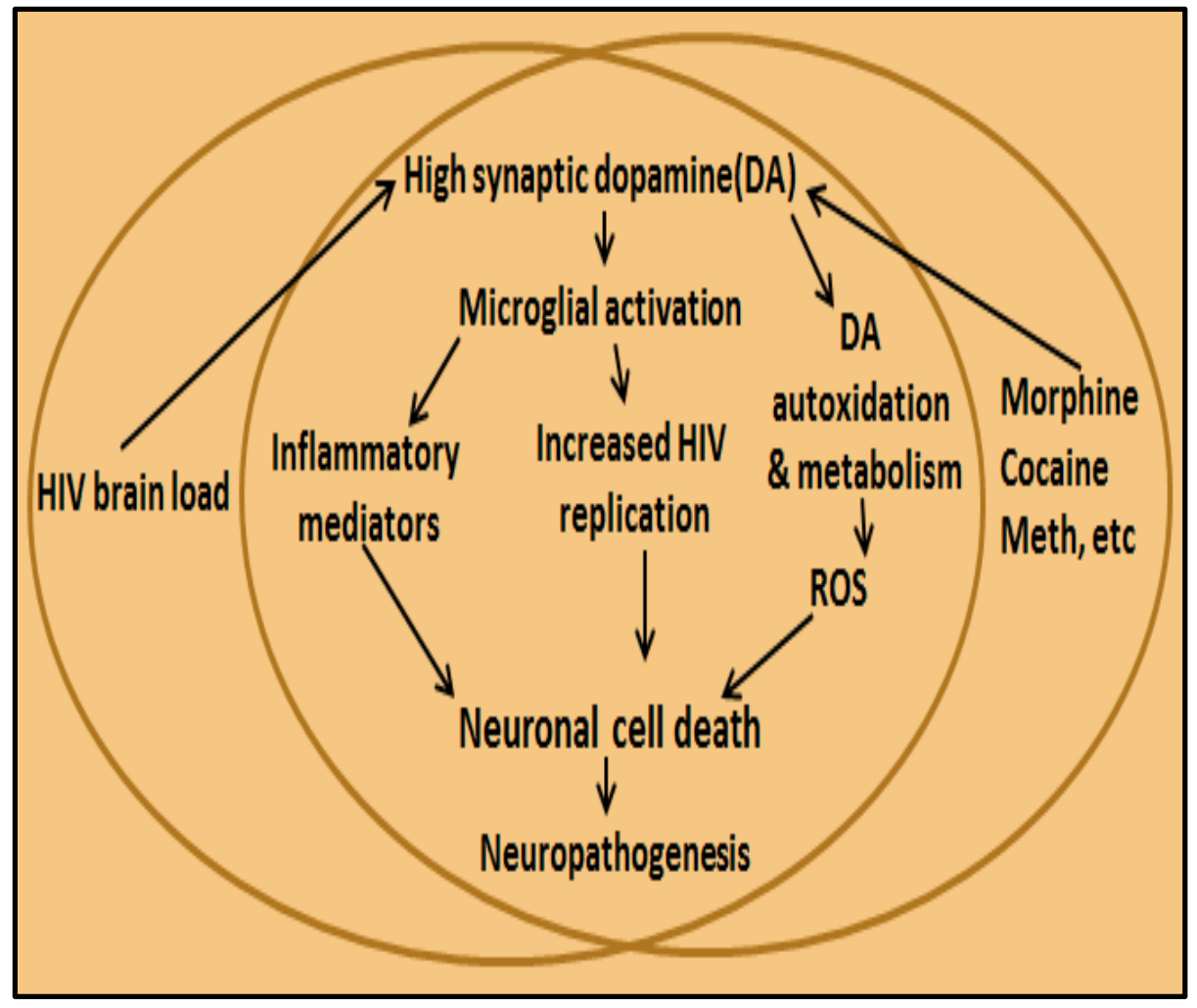

Figure1. Simplified overview of the common pathways involved in HIV and Recreational drugs induced neuropathogenesis. 


\subsubsection{Neurobiology of morphine addiction and effect on HIV infection:}

Morphine, a $\mu$ opioid receptor agonist is a highly potent analgesic drug. It is highly addictive resulting in rapid development of tolerance, physical and psychological dependence (Coller and Hutchinson, 2012). Several attempts have been made in the past to understand the neurobiology of opiate addition (Nath et al., 2002; Burdo et al., 2006; Hauser et al., 2007; Ferris et al., 2008; Rogers, 2011). There are various hypotheses that describe morphine addition that includes the alteration in affinity or number of opioid receptors (Simonato, 1995), changes in specific brain regions, alterations in neurotrophin signaling pathways that regulate the non-adrenergic response of Locus Coeruleus (LC) neurons to opiates (Hatami et al., 2007) and role of glutamate receptors (Inoue et al., 2003). Thus, morphine tolerance and addiction involve intricate mechanism involving dopaminergic, noradrenergic and glutamatergic neurons. Due to this complexity, opioid dependence is proposed to involve multicellular event, where individual neurons differentially adapt based on the signals they receive and the second messengers and genetic programming of the cell (Simonato, 1995). The exact mechanism by which opiate acts as a cofactor for HIV infection is not clear. However, it is reported that $\mu$ opioid ligands act synergistically with HIV proteins (tat; transactivator and gp120) to potentiate the HIV-related neurotoxicity. Further, morphine upregulates the expression of HIV entry coreceptors CCR3 and CCR5 on astrocytoma cells, which increases viral binding and entry into the cells and promotes viral replication (Stefano et al., 1996). In recent years, the role of astrocytes and microglia in the neuropathogenesis of HIV is becoming 
increasingly evident. Therefore, use of a $\mu$ opioid receptor antagonist in addition to ART could be of therapeutic importance and may provide significant benefits in treatment of HIV infected subjects who are opiate users. CTOP (D-Pen-Cys-TyrDTrp-Orn-Thr-Pen-Thr-NH2) is a highly selective and potent $\mu$ receptor antagonist; however it is impenetrable through the Blood Brain Barrier (BBB). The current study proposes to bind CTOP to the magnetic nanoparticles and target to the brain using an in vitro BBB model.

\subsection{Problems of neuroAIDS treatments:}

\subsubsection{Limitations of current treatments:}

Highly active anti-retroviral therapy (HAART) has been successfully implemented for management and prevention of AIDS progression. Antiretroviral (ARV) drugs recommended by WHO for HAART formulations belongs to seven classes: Nucleotide Reverse Transcriptase Inhibitors (NtRTI), Nucleoside Reverse Transcriptase Inhibitors (NRTI), Non- Nucleoside Reverse Transcriptase Inhibitors (NNRTI), Protease Inhibitors (PI), Fusion Inhibitors (FI), Integrase Inhibitors (Inl), and CCR5 antagonists (AIDSinfo-NIH, 2012). Basically, combinations of three or more class of antiretroviral (ARV) drugs are formulated for HAART regimens. With the proper HAART treatment plasma viral load can decline below the detection limit (Douce et al., 2012) and median life expectancy of AIDS patients may also rise by tenfold (Vyas et al., 2006; Mallipeddi and Rohan, 2010). In fact, HAART has resulted in remarkable decline in the mortality 
rate of AIDS Patients during the last decade and it is predicted that $50 \%$ of HIVinfected people will cross the age of 50 by 2015 (Smith, 2005, Holt et al., 2012). Undoubtedly, as a result of HAART, this lethal disease has been transformed into a chronic pathology. Nevertheless, little irregularity or interruption of HAART treatment lead to resurgence of suppressed viral replication (Douce et al., 2012) and so, challenge of complete restriction or elimination of progression of HIV infections still exist.

A dramatic decrease in morbidity of many AIDS related symptoms is noticed following the HAART treatment. In the same line, occurrence of some of the neuronal disabilities has also been remarkably declined (e.g. HIV associated dementia (HAD) and symptomatic distal sensory polyneuropathy has been reduced to less than 7 and $10 \%$ of affected people respectively) (Antinori et al. 2007; Woods et al. 2009; Vivithanaporn et al., 2010). Nevertheless, a concomitant rise in the other form of CNS dysfunction such as minor cognitive impairments/motor disorders has widely been noticed in the patients on HAART regimes (Fischer-Smith and Rappaport, 2005; Ghafouri et al., 2006; Kraft-Terry et al., 2010; Wong et al., 2010). This resulted in an increase in the cumulative occurrence of HIV associated neurocognitive complications. Vivithanaporn et al., (2010) reported that during the decades of $1998-2008$, at least $25 \%$ of HAART treated patients developed one or other neurological syndrome. Thus, burden of HIV associated neurological disorders prevail on larger scale. This reduced efficacy of current HAART regimens for treatment of increased incidence of 
neuroAIDS can be attributed to many reasons. Firstly, these treatments are not targeted for inflammatory cascades underlying any of the HIV-associated neuronal disorders. Thus, HAART does not have direct effect on the HIV associated inflammatory degeneration (Rumbaugh et al., 2009). Secondly, inadequate reachability of $A R V$ drugs across the brain barriers has minimum effect on the resting viral loads in the brain hideout. This may result in gradual generation of resistance viral strain against HAART as has been seen in some of the infected populations (Vella and Palmisano, 2005; Amiji et al., 2006; Kozal, 2009; Griffiths, 2009; Nijhuis et al., 2009; Gupta and Jain, 2010). Third, and importantly, inadequate CNS reachability of ARV drugs, which is also the main obstacle towards treatment of neuroAIDS, is caused due to impermeability of drugs across the brain barriers. This can be attributed to properties of brain barriers that make it ultra-selective permeable for both, endogenous compounds and xenobiotic molecules as well. Additionally, ARV drug's short half-life and low bioavailability, due to extensive first pass metabolism including gastrointestinal degradation, may also add to their insignificant arrival in the CNS (Gupta and Jain, 2010; Wong et al., 2010). Moreover, emergence of various side effects and cost of HAART may also result in cessation of treatment (Nolan et al., 2005; Douce et al., 2012). Overall, the basic problem of HAART failure in treatment of neuroAIDS lies in the structural and functional complexity of brain barriers. 


\subsubsection{Barriers of CNS:}

The organizational uniqueness of CNS is featured by three structural barriers, namely, the BBB, blood-cerebrospinal fluid barrier (BCSFB), and the Cerebrospinal fluid-brain barrier (CSFB). In particular, the BBB and BCSFB are very special anatomical features because they safeguard the brain from the periphery, respectively by means of tightly junctioned brain microvessel endothelial cells (BMECs) and choroid epithelial cells. These tightly packed structures possess very low and selective paracellular permeability. In contrast, CSFB may not be considered as an anatomical barrier because it is structured by loosely linked ventricular ependymal cells and can readily allow reversible diffusion of solutes from ventricular cerebrospinal fluid (CSF) to brain parenchyma or vice versa (Enting et al., 1998; McGee et al., 2006; Johanson et al., 2011).

The BBB function as the interface that separates the brain parenchyma (CNS) from the blood stream (peripheral circulation). It is an extensive, continuous, fenestrationless, and almost impermeable barrier of tightly junctioned BMECs along the capillaries lining throughout the cerebral microvasculature. The tightness of this transendothelial junction is $50-100$ folds higher than the peripheral vessels, giving an electrical resistance of $1500-2000 \Omega \mathrm{cm}^{-2}($ Crone and

Christensen, 1981; Butt et al., 1990; Gonzalez-Mariscal et al., 2003; Weksler et al., 2005; Hamilton et al., 2007; Wang and Bordey, 2008). This indicates the severity of opposition-intensity to the passage of molecules from entering the 
cerebral space. The structural sophistication of the BBB is further compounded by persistent and intimate contact of BMECs to other neuronal cells, mainly pericytes and perivascular astrocytes (Hawkins and Davis, 2005; Abbott et al., 2006). The integrity of tight junctions is maintained by three main tight junction transmembrane integral proteins, occludin, claudin and junction adhesion molecules and many cytoplasmic accessory proteins, such as zonula occludens, cingulin, 7H6 antigen, etc. (Citi et al., 1988 ; Hirase et al., 1997; Haskins et al., 1998; Denker and Nigam, 1998; Furuse et al., 1999). Additionally, BMECs possess few pinocytotic vesicles and its mitochondrial content (both quantity and volume) is also high which, respectively, limit the transcytosis and fuel the increased demand of transport activity associated with the endothelial influxefflux pump (Enting et al., 1998). Only selected molecules necessary for ideal functional efficiency of the brain such as certain amino acids, monocarboxylic acids, amines, sugars, purine bases, hydrophilic molecules like $\mathrm{O}_{2}$ and $\mathrm{CO}_{2}$, etc. is actively transported via mechanisms such as carrier mediated transport, fluidphase endocytosis, receptors- or absorptive -mediated endocytosis (King and Johnson, 1985; Gonatas et al., 1984; Fishman et al., 1987; Dehouck et al., 1994; Descamps et al., 1996; Tsuji and Tamai, 1999; Tamai and Tsuji, 2000; Wolka et al., 2003; Yousif et al., 2007; Hamilton et al., 2007; Zhang, 2010). Many substrate specific transporters such as monocarboxylate transport system, glucose transporter-1, insulin receptor, transferrin receptor, ceruloplasmin receptor, etc are present on the BMECs (Wong et al., 2012). Also, certain neurotransmitters and small lipophilic xenobiotics or endogenous molecules up to 
molecular weight of 600 Dalton can freely diffuse transcellularly across the BBB (Pardridge, 2001). However, all these mode of selective permeability and transportation of small lipophilic or other drug molecules across the BBB provide very little or no benefit for the management of most brain diseases. Transportation of small or large drugs in the CNS, in overall, is also critically affected due to functional sophistication of the BBB. Regardless of the drug's ability or inability to permeate the BBB, their active percentage in the CNS remains below the pharmacological significant level in most cases including many ARV drugs. Towards this end, endothelium of the BBB is equipped with large spectrum of influx-efflux receptors/proteins that can actively transport molecules such as nutrients, metabolites, hormones, neurotransmitters, peptides, drugs, etc in or out of the brain. These transporters have been classified into two main groups, namely, ATP-binding cassette (ABC) transporters and solutecarrier (SLC) superfamily. Major ABC transporters and SLC carriers that affect drug delivery across the BBB are P-glycoprotein (P-gp), breast cancer resistance protein (BCRP), multi-drug resistance-associated proteins (MRPs), organic cation transporter (OCTs), organic anion transporters (OATs), organic anionstransporting polypeptide (OATPs), equilibriative and concentrative nucleoside transporters (ENTs and CNTs), system L-transporters, etc. Many of these carriers have been shown to affect the distribution of ARV drugs across the BBB (Ronaldson et al., 2008; Varatharajan and Thomas, 2009). An anti-HIV drug may serve as substrate, inhibitor or both for different influx-efflux transporters or, in other words, these transporters possess overlapping specificity for ARV drugs. 
Such as, Abacavir, an NRTI, is a substrate for ABC transporters, P-gp and BCRP; and simultaneously, it functions as an inhibitor of other $A B C$ transporters, MRP-1, 2 and 3 (Pan et al., 2007; Shaik et al., 2007; Weiss et al., 2007; Giri et al., 2008). Similarly, Nelfinavir, a PI, is substrate for P-gp and inhibitor of BCRP and two SLC superfamily transporters, OCT-1 and 2 (Kim et al., 1998; Gupta et al., 2004; Jung et al., 2008). While being a substrate of efflux transporters causes own inaccessibility to the target, as inhibitor of influx transporters it blocks the CNS entry of corresponding useful substrates. Further, as inhibitors of efflux transporters, an ARV drug may influence the targeted delivery-kinetics of corresponding drug substrate and this may be reason for positive or negative drug interaction during successful or failed recipe of a combined ART therapy. BEMCs also possess enzymatic barrier for metabolization of undesirable neuroactive substances recruited through blood (Minn et al., 1991; Brownson et al., 1994). Elevated expressions of various enzymes such as $\mathrm{y}$-glutamyl transpeptidase, aromatic acid decarboxylase, alkaline phosphatase, etc are found in cerebral microvessels. Metabolism-dependent luminal or abluminal expression of these enzymes significantly affects the dynamics and kinetics of xenobiotics in the brain (Betz et al., 1980; Hau, 2005). Thus, in order to maintain the brain homeostasis, various structural and functional uniqueness of the BBB allow exogenous molecules at zero level or far below the pharmacological significant amount. Therefore, the BBB may be considered as the primary impediment that prevents drug penetration into the CNS. 
The BCSFB, at the choroid plexus in ventricles, also restricts flux of ions and molecules from the blood into the brain. Anatomically, tightly junctioned monolayer of choroid epithelial cells surrounds highly permeable fenestrated endothelium of choroid capillaries. This creates a barrier between the extracellular blood filtrate from these capillaries and the CSF in the brain ventricular spaces. The epithelium of this barrier possesses a special combination of basal labyrinth contacting the blood filtrates and profuse apical microvilli towards the CSF. This unique structural arrangement affords necessary surface area for appropriate transport physiology of solute and water exchange across the BCSFB (Enting et al., 1998; McGee et al., 2006; Johanson et al., 2011). The functional sophistication of the BCSFB is similar to that of the BBB in many ways. As such, dense mitochondrial content and various influx/efflux receptors and transporters belonging to $A B C$ and SLC superfamily are also present in choroid epithelial cells (Varatharajan and Thomas, 2009; Wong et al., 2012). However, BCSFB is quantitatively more permeable and possesses unique transportability for many compounds and molecules such as $\mathrm{Ca}^{2+}$, iodide, homovanillic acid etc (Siegel et al., 1999). Various transportation processes such as low capacity transcellular pinocytosis/exocytosis, limited facilitated or nonfacilitated paracellular diffusion, and receptors-transporters mediated active passage have been demonstrated in the BCSFB. Additionally, presence of few incomplete junctions at this barrier is putatively believed to allow protein/peptides movement within a limit. Importantly, brisk blood flow at the choroid plexus $(\sim 10$ times more than the brain) increases the overall concentration of metabolites in 
the extracellular blood filtrate from capillaries leading to increased passage probability across the BCFB via relevant transportation routes (Johanson et al., 2011). Nevertheless, most xenobiotics remain impermeable to the BCSFB. Even most of the transportable metabolites could be secreted in the ventricular CSF by epithelial cells and must diffuse into brain parenchyma to be effective. Notably, the CSF from both, choroidal (epithelium) and extrachoroidal (from brain capillaries and ventricular wall) secretion is deposited in the ventricular spaces. Basically, the CSF serves as "sink" that collects metabolic waste and other molecules from the brain in a gradient dependent manner and releases them over the brain. This helps to maintain the steady-state concentrations of various endogenous molecules and xenobiotics that penetrate into the brain or CSF (Davson et al., 1961). The CSF from ventricles passes into cerebral- and spinalsubarachnoid spaces where it is separated from the direct blood contact via the tight arachnoid membrane. Arachnoid membrane contains arachnoid villi for absorption and unidirectional exit of the CSF and its metabolites into the venous sinuses over the brain surface. The CSF in the ventricular and subarachnoid spaces may also reversibly diffuse into brain interstitium through loosely linked ependyma of CSFB and pia-glial membranes respectively. Diffused compound from CSF to brain interstitium can reach to parenchyma (neurons and glial cells) or/and may face the BBB along the cerebral microvasculature (Johanson et al., 2011). However, diffusion of such metabolites to the parenchyma is remarkably hampered due to rapid rate of the CSF turnover ( $\sim 4$ times per day) (Davson and Segal, 1996; Siegel et al., 1999). The CSF convection and bulk flow towards the 
exit rout through arachnoid villi is much faster than drugs diffusion from the CSF to the brain parenchyma leading to rapid removal of CSF metabolites. Also, larger distance between the CSF and brain interstitial fluid decreases the diffusion process (Pardridge, 2011). Thus, presence of drugs in the CSF does not guarantee its passage to the brain. Large molecular weight molecule and hydrophilic compounds are more frequently released due to sink effect. As such, azidothymidine (AZT), an ARV drugs, can enter the CSF probably either as a result of their bulk flow movement or using the choroid thymidine transporter; however, it never reaches to the brain (Wu et al., 1992). Overall, we see that with the novel molecular, structural, and functional arrangements, BCFB can be considered as the secondary barrier that prevents drug penetration into the CNS.

\subsection{Advantages of nano-scale technology in drug-delivery:}

Nanotechnology harvests the unique physicochemical parameters of materials at a nanometer size range. Few of the intrinsic properties of nanoparticles such as higher specific surface area and increased circulation time have shown remarkable potential for their use as novel drug carrier. Also, other properties like biocompatibility, surface charge, hydrophobicity, and crystallinity are among the fundamental considerations for selection of a nanoparticle in the field of medicine (Semete et al., 2010). The concept of nanodrugs revolves around development of "target-specific, effective, safe and controllable" drug-delivery method which is need of the hour. Basically, drugs, alone or in association/combination with target-specific molecules, are enclosed 
in or absorbed on nanoparticles for better efficacy and lesser side effects (Liu et al., 2010).

Superiority of the nano-drug delivery methods could be attributed to combinations of its various features. Firstly, a dramatic increase in the bioavailability of drugs can be achieved through nano-drugs or nano drugdelivery carrier. As such, a significant amount of orally administered nanocapsulated drugs $(<100 \mathrm{~nm})$ escape the portal blood circulation route avoiding the reticuloendothelial digestion; rather they are passed to systemic circulation via intestinal lymphatic transport resulting in remarkable reduction in the first pass hepatic metabolism which enhance their quantity and duration of bioavailability. Further, because of the ability to freely flow into capillaries and remarkable increase in blood circulation time, nanoparticles can travel to tissues in every nook and corner of the body (McNeil, 2005; Desai et al., 1996; 1997). The nano-size particles are compatible for easy intracellular uptake and can even travel across different physiological barriers such as BBB, stomach epithelial, etc (Koziara et al., 2003). The increased circulation time and higher cellular uptake of nanoparticles is greatly influenced by their surface charge and hydrophobicity/hydrophilicity (besides size). While coating of nanoparticles with positively charged molecules such as chitin enhances their attachment to negatively charged surface of cells, coating with hydrophilic compounds (e.g. polyethylene glycol, pluronics, etc) circumvent opsonization resulting in longer blood circulation time (Freiberg and Zhu, 2004; Mohanraj et al., 2006; Park et 
al., 2010). The hydrophobic/hydrophilic nature of nanocarriers also affects the solubility of weaker hydrophilic drugs, and thus in turn influences their bioavailability (Semete et al., 2010). Moreover, the larger surface to volume ratio of nanoparticles allows higher drug loading and dissolution rate influencing the bioavailability (Soppimath et al., 2001; Kondo et al., 1993; Semete et al., 2010). Additionally, crystallinity of many nanoparticles (e.g. polymers) significantly affects their degradable speed which influences the biological halflife of associated drugs (Izumikawa et al., 1991; Mahato, 2007).

Secondly, nano-drugs possess comprehensive advantages in context to the drug release kinetics. The increased specific surface area of nanoparticles enhances the drug loading ability. Higher amount of drugs in nano-carrier results in initial burst release and then followed by a constant slow release, which affect the kinetics and minimize dose frequency (Soppimath et al., 2001; Kondo et al., 1993). Similarly, crystallinity of materials affects their dissolution characteristics - the amorphous region degrades faster in compare to crystalline region. Thus, release kinetics of associated/combined drug is affected (Izumikawa et al., 1991; Mahato, 2007). Furthermore, surface charge and hydrophobicity of nanoparticles or coating materials play significant role in drug kinetics. These properties of nanoparticles greatly affect their molecular composition and so as their degradation rate is influenced (Semete et al., 2010). The increased blood circulation time due to hydrophobic coating prolongs the associated drug release. Also, hydrophilic coating reduces the 
dose frequency of the poor soluble drugs due to their improved solubility (Blume, 1993; Vert and Domurado, 2000, Immordino et al., 2006).

Third and last, the feasibility of selective targeting can be significantly improved by the nano-drugs. This, in turn, can improve the drug efficacy and side effects can be minimized as well. Nano-drugs or nano-carriers can be molded for both, passive and active targeting. The reduced first pass hepatic metabolism and increased blood circulation time of nanoparticles makes them suitable for the purpose of passive targeting. The application of passive targeting of lymph nodes (Maeda et al., 2000; Mitra et al., 2001; Briz et al., 2003; Gunaseelan et al., 2010). In active targeting, drugs or carriers are combined with target efficiency of nanoparticles has been successfully demonstrated in the case of enhanced permeability and retention effect in targeting tumors of enterohepatic circuit and HIV infections moieties or vector molecules that can recognize and bind to a specific target sit. Thus, direct administration of a drug into an affected organ or tissue can be achieved. Various substances like antibodies, peptides, hormones, polysaccharides, lipoproteins, etc can be used as targeting moieties. Thermal- and $\mathrm{pH}$-sensitive targeting molecules (e.g. $\mathrm{N}$-isopropylacrylamide) and suitable adjuvant can also be part of active targeting nano-drug carriers (Chung et al., 1998; Kohori et al., 1998; Meyer et al., 1998; Yoo et al., 2002; Sershen et al., 2000; Liu et al., 2010). 


\subsection{Nanomedicines for neuroAIDS treatment:}

A complete lack of ARV therapies for $\sim 40 \%$ of AIDS patients (WHO, 2012) and further, ineffectiveness of HAART in treatment of HIV-associated neurological syndromes has molded the neuroAIDS as a consistent global problem. In the wake of fact that more than $98 \%$ of small and large drugs are unable to cross the brain barriers (Pardridge, 2007), which is believed to be main impediment in the cure of neuroAIDS, several strategies are being experimented to administer the desired therapeutic levels of anti-HIV drugs across those barriers. Transcranial drug delivery (focused ultrasound and microbubble approach to disrupt the BBB), transnasal drug delivery (direct access to CNS from nasal cavity via olfactory neurons and avoid fist-pass metabolism), disruption of BBB using pharmacological agent (etoposide and cisplatin), hyperosmotic solutions (mannitol and urea), prodrugs approach (lipidization of ARV molecules and fusion of drugs with cell-penetrating peptides or antibody specific to $B B B$ receptors), and inhibition of $A B C$ transporters (P-gp) are few approaches with potential to deliver ARV drug across BBB (Wong et al., 2010; Nair and Saiyed, 2011). However, these strategies have less strength than limitations which restrict their use as common and novel drug delivery method. An effective drug delivery method or diagnostic agent must have systemic administration ability i.e., majority of therapeutic agents should be delivered to the target site while non-target site should get minimal drug exposure. Complying with this 
notion, practice of nanotechnology in medicine has shown exciting prospect for development of novel drug delivery system.

\subsection{Functional nanovehicles for prevention and treatment of neuroAIDS:}

Improved drug delivery across brain barriers is essential for the management of neuroAIDS. In this context, applications of various nanocarriers have generated a promising trend for the better ARV drug distribution to the CNS. Schemes of CNS drug delivery using nanovehicles can be broadly classified based on their passive or active targeting ability (Wong et al., 2010). Approaches involving the passive targeting can result in accumulation of higher concentration of drug at endothelium of the BBB. This local gradient difference may allow the drug penetration by passive diffusion. Also, trafficking via non-receptor mediated endocytosis (e.g., macropinocytosis) may enhance the cellular drug uptake. Actively targeted drug trafficking can be possible via receptor mediated endocytosis when periphery of nanocarriers is tagged with ligand molecules matching to specific cell receptor (Pelkmans et al., 2001; Amyere et al., 2002; Kaplan et al., 2005; Khalil et al., 2006). Nanocarriers can also be tagged/ loaded with specific efflux transporters inhibitors or blocking agent which can result in increased drug concentration across the BBB (Liu et al., 2010). Several nanocarrier systems such as liposomes, dendrimers, different nanoparticles, micelles, etc. have been intensively explored (Figure 2) and approaches for their improvement are 
under investigation. Recently, applications of magnetic nanocarriers and monocytes/macrophage based nanoformulations have gained considerable interest for the treatment of neuroAIDS.

\subsection{1. polymeric nanovehicles:}

Acrylic and polyester polymers are the most studied synthetic polymeric compounds as nanocarriers for CNS drug delivery. Poly (butyl cyanoacrylate) (PBCA), an acrylic polymer, have been extensively explored for this purpose. PBCA possess rapid in vivo degradation ability which can minimize their longer accumulation and, in turn, can prevent the brain from potential polymeric toxicity (Wong et al., 2012). The lipophilic property of PBCA makes it suitable for loading of various kinds of compounds with hydrophilic property and weak/low basicity (Alyaudtin et al., 2001; Gao et al., 2006). Without causing any permanent physical harm to $\mathrm{BBB}, \mathrm{PBCA}$ nanoparticles are able to deliver an improved amount of ARV drugs in both brain tissues and CSF. Kuo and Chen (2006) reported that the use of PBCA nanoparticles enhance the in vitro BBB permeability of ARV drugs zidovudine and lamivudine by $8-20$ and $10-18$ fold, respectively. In the same study, application of other acrylic polymer nanoparticle, methylmethacrylate-sulfopropylmethacrylate (MMSPM), showed $100 \%$ rise in the BBB permeability of zidovudine and lamivudine. Additionally, PBCA and MMPSM coated with PS-80 (a tensoactive agent) were used for the delivery of ARV drugs, stavudine, delaviridine, and saquinavir, in in vitro BBB model. It was found that permeability of these three drugs was enhanced by 
-12-16 and 4-11 folds with PBCA and MMSPM formulations respectively (Kuo and Su, 2007). Increase in the BBB permeability of acrylic polymers are facilitated by receptor mediated transcytosis which is triggered by binding of apolipoproteins (adsorbed on the surface of polymeric nanoparticles) to the low density lipoprotein receptors on BMVECs (Kim et al., 2007). Besides this transcellular pathway, acrylic polymers can also use the paracellular route via reversible disruption of BBB for short period of time (Alyaudtin et al., 2001; Rempe et al., 2011).

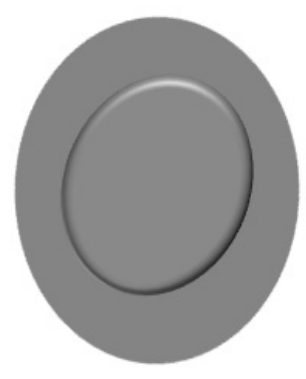

(A)

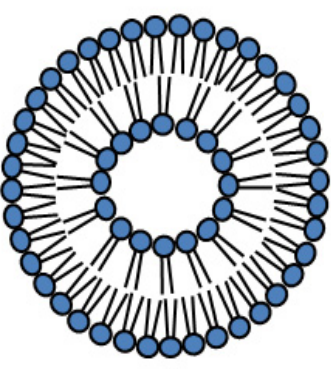

(E)

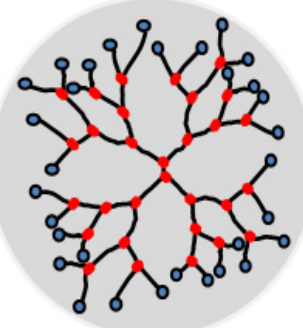

(B)

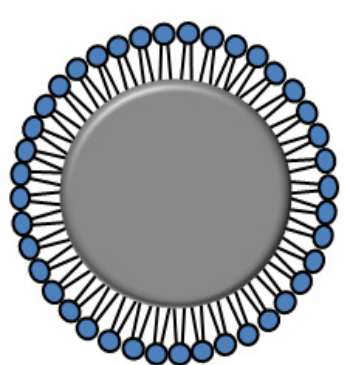

(F)

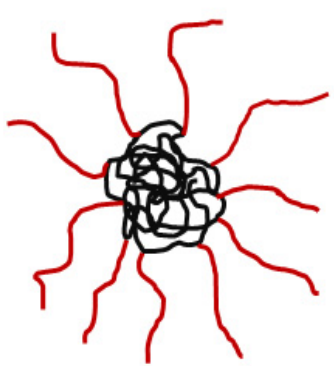

(C)

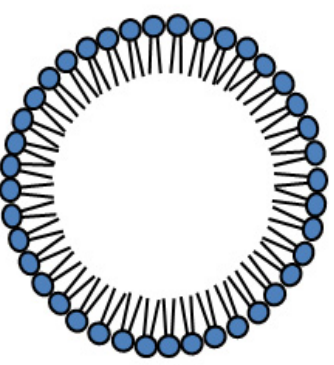

(G)

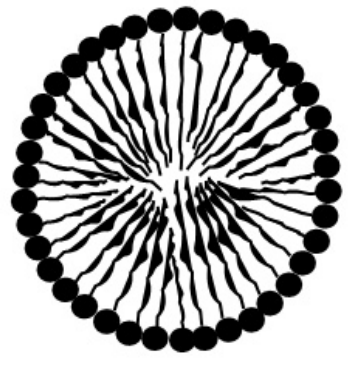

(D)

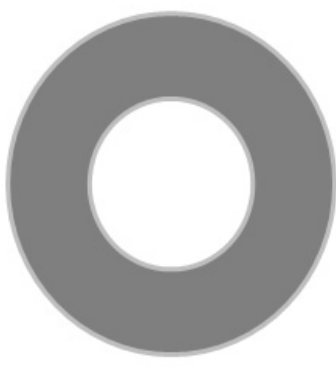

$(\mathrm{H})$

Figure 2: Nanoparticles used for the delivery of ARV drugs across BBB. (A) Polymeric nanoparticle. (B) Dendrimer nanoparticle. (C) Polymeric micelle. (D) Nonpolymeric micelle. (E) Liposomes. (F). Solid lipid nanoparticles. (G). Lipid nanoemulsions. (H) Lipid nanocapsules. 
Despite these merits, application of polymeric nanoparticles is restricted because they are not ideal for the delivery of polar/ionic compounds (Wong et al., 2010). Furthermore, process of PBCA degradation can produce toxic formaldehyde by-products (Olivier, 2005). Thus, other polymers like polyesters are considered a safer choice for CNS drug delivery. Two polyester, polylactide (PLA) and poly(lactide-co-glycolide) (PLGA) have been approved by United States Food and Drug Administration for human use (D'Souza and DeLuca, 2006). These highly versatile biocompatible polyesters are degraded into glycolic acid and lactic acid which are converted into water and carbon dioxide via TCA cycle and eventually eliminated from the body (Yoo et al., 2005; Makadia and Siegel, 2011). Importantly, injection of these polyesters induces negligible and transient inflammatory response (Athanasiou et al., 1995; DechyCabaret et al., 2004). Variety of drugs of both hydrophilic and hydrophobic nature can be entrapped on the matrix of PLA and PLGA. In addition, drugs entrapment can be tailored for sustained release for longer time (Zhang and Feng, 2006). Surface modifications of these polyester polymers such as PEGlyation (attachment of poly(ethylene glycol), agglutinin coating, alginate embedding, etc., have been strongly recommended for delivery of therapeutic dose across the BBB. Both, PLA and PLGA with certain modifications have been shown to be useful for the improved brain delivery of many non-ARV drugs such as dexamethasone, vasoactive intestinal peptide, superoxide dismutase, etc (Kim and Martin., 2006; Gao et al., 2007; Reddy et al., 2009; Liu et al., 2012). Study on ARV drugs by Destache et al., (2010) demonstrated that 
nanoformulations of ritonavir, lopinavir, and efavirenz with PLGA can maintain a sustain peak of about 28 days in mice brain which is limited to only 2 days with free drugs. Similarly, Rao et al., (2008) demonstrated that at two weeks postadministration, PLA nanoparticles in conjugation with Tat peptides could result in 800 fold higher level of ritonavir in mouse brain in compare to drug delivered in solution. It should be noted that the cell penetration ability of Tat peptide makes it a natural accessory for carriers used for drug delivery across BBB. Along with synthetic polymers, natural polymers such as albumin, chitosan, alginate, gelatin, collagen, etc. are also being explored as the potential nanocarrier for brain drug delivery (Semete et al., 2010). Al-Ghananeem et al (2010) investigated the potential of chitosan for delivery of ARV drugs via both, intravenous and intranasal route and found a significant improvement in the level of didanosine in brain and CSF of rat.

\subsubsection{Dendrimer nanovehicles:}

Dendrimers are basically globular or spheroidal structures made up of controlled repeats of monomer units branched around a central core (Figure 2B) (Svenson et al., 2009; Wong et al., 2010). They can be engineered in the size range of $10-100 \mathrm{~nm}$ and may contain many reactive functional end groups, which make them potent for drug delivery systems (Semete et al., 2010). Dendrimers may also contain internal void spaces. Thus, both encapsulation (in void space) and conjugation (with reactive end groups) of compounds with different polarity can be possible with dendrimers. Though more than 100 types 
of dendrimers exist, five main classes used for medicinal purposes are: Polyamine amine, Polypropyleneimine (PPI), Phosphorus, Carbosilane, and Polylysine dendrimers. However, Phosphorus dendrimers has never been used for HIV research (Dzmitruk et al., 2011). Similar to polymeric nanoparticles, dendrimers have been mostly studied for brain delivery of anti-cancerous drug. Nevertheless, Jiménez et al., (2010) investigated the potential of 2G-NN16 dendrimers (a Carbosilane dendrimer) in in vitro BBB model for delivery of antiviral (HIV) siRNA. This siRNA/2G-NN16 dendriplexes showed permeability across the in vitro BBB and caused a significant reduction in the viral replication. Most of the ARV study involving dendrimers in HIV research has been restricted to different cell types such as, macrophages, dendritic cells, MT2 cells, etc (Dutta and Jain, 2007; Dzmitruk et al., 2011) and thus, more in vitro and in vivo BBB investigations are required before their use for CNS delivery of ARV drugs. The limited application of dendrimers can be attributed to their complex synthesis process and inconsistent and premature drug release kinetics (Wong et al., 2010). The drug release mechanism is also not clear, though some report suggest toward transcytosis through the BBB (Dzmitruk et al., 2011). Additionally, polycationic surface groups of dendrimers proved to be toxic for negatively charged cell membranes resulting in cell death (Bawarski et al., 2008; Pion et al., 2010). 


\subsubsection{Micelles nanovehicles:}

Micelles are self-aggregated assembly of amphiphilic molecules dispersed in aqueous media (Kabanov and Alakhov, 2002; Wong et al., 2006). The diameter of micelle particles may vary from 1-50 nm (Semete et al., 2010). Particles are assembled in such a way that there is an inner hydrophobic core and the hydrophilic heads of amphiphilic molecules are exposed outside (Figure $2 \mathrm{C}$ and $\mathrm{D}$ ). The inner core serves as the encapsulation space competent for the better solubilization of poor water-soluble and lipophilic compounds (Jones and Leroux, 1999; Gaucher et al., 2005; Bae and Kataoka., 2009; Wong et al., 2010). Three types of amphiphilic molecules, namely, block-copolymers, surfactants and polymer-lipid conjugates are used for formation of micelles (Trivedi and Kompella, 2011). However, pluronic block-copolymers has been the most studied micelles types for CNS drug delivery. Pluronic micelles demonstrate zero toxicity to the BBB and can inhibit efflux transporters such as P-gp, MDR1, etc which, in turn, increase their substrate permeability (Batrokova et al., 2003; Spitzenberger et al., 2007; Shaik et al., 2008). Notably, many ARV drugs are substrates for efflux transporters/receptors of BBB. Thus, pluronic micelles can serve as both, drug carrier and efflux inhibitor and have been demonstrated to be valuable for CNS delivery of ARV drugs. Batrokova et al (1999) showed that exposure of pluronic P85 enhance permeability of ritonavir across in vitro BBB. Similarly, in vivo experiment by Spitzenberger et al (2007) demonstrated that administration of pluronic P85 alone or in combination with 
ART (zidovudine, lamivudine, and nelfinavir) resulted in $78-92 \%$ reduction in the p24 expressing monocyte-derived macrophages (MDM) from mouse brain in compare to $62 \%$ of only ART treated group at two weeks post-inoculation of HIV. Additionally, Sharma and Garg (2010) suggested that micelles may be tailored for highly selective active targeting by tethering hydrophilic block to ligands specific to HIV reservoir receptors such as lecitn. Nevertheless, instability of the non-cross-linked pluronic micelles remains a matter of concern because it may reduce the circulation time resulting in premature drug release (Trivedi and Kompella, 2010).

\subsubsection{Liposomes nanovehicles:}

Liposomes are the first and probably the most applied drug delivery carrier (Langer, 1976; Costantino et al., 2009; Wong et al., 2012). They can be defined as auto-spontaneously arranged unilamellar or multilamellar, spherically closed colloidal vesicles made up of amphipathic phospholipid bilayer membranes surrounding an aqueous core. While one hydrophilic head of phospholipid bilayer is exposed to outside, the other is in contact with vesicle core (Figure 2E). Thus, hydrophobic group of the bilayer is protected from the aqueous environment. This unique character of liposomes allows loading of both hydrophilic (encapsulated in aqueous core) and hydrophobic/lipophilic (incorporated into the bilayer of phospholipid) compounds (Sharma \& Sharma, 1997; Voinea et al., 2002; Carvalho et al., 2011). Depending upon the processing methods and constituent, size of liposomes may go up to $\mathrm{mm}$; 
however, it can be restricted to a minimum of $20-30 \mathrm{~nm}$ which is ideal for a nanocarrier (Wong et al., 2010). Surface of liposomes can be engineered for active targeting by applying surface charge modifications (Makino and Shibata, 2006) and/or conjugation of antibodies/ligands specific to diseased cells or tissues including brain and CNS (Desormeaux and Bergeron, 1998; Allen et al, 2002; Kreuter, 2004). Additionally, modifications such as PEGlyation can improve the inherent poor stability of conventional liposomes (Koukourakis et al., 2000; Jain, 2008; Bertrand et al., 2010) and can also reduce their uptake by reticuloendothelial system resulting in improved plasma circulation time (Gunaseelan et al., 2010). Lipid composition may also be tweaked for better stability and circulation rate (Vitas et al., 1996; Katragadda et al., 2000). Different types of liposomes used so far for the delivery of anti-HIVIAIDS drugs can be broadly categorized into ionic-, immune- and sterically-stabilizedliposomes (Lanao et al., 2007). The rationale of using liposomes for ARV drugs is based on the fact that mononuclear phagocytic system recognizes conventional liposome as foreign body; and since monocytes and macrophages are HIV reservoirs and can travel to brain, an improved efficacy of drugs can be achieved (Prior et al., 2002). Kim et al., (1990) demonstrated that half-life of intraventricularly administered, liposome-encapsulated zalcitabine in the brain of Sprague-Dawley rat increase to $23 \mathrm{~h}$ as compared with $1.1 \mathrm{~h}$ for the unencapsulated drug. In the same line, Dusserre et al., (1995) showed that liposomal encapsulation could enrich the rat brain with about 13 times more foscarnet - a salvage therapy for multi-drug resistant AIDS patients - in 
compare to its solution. Further, the superiority of CNS targeting ability of liposomes-loaded AZT-myristate (prodrug of AZT) was studied by Jin et al (2005). It was shown that, with about $98 \%$ encapsulation efficiency and longer half-life, a higher concentration of AZT was found in the brain and other organs of rats. Potential of liposomes have also been evaluated for management of HIV-related opportunistic infections which is critical for the HIVIAIDS patients. Several fold increase in the concentration of amphotericin B (drug for fungal infections in HIV patients) was demonstrated when liposomes tethered with RMP-7 (Bradykinin B2 receptor agonist) were used for delivery across in vitro rat-BBB model (Zhang et al., 2003). Despite these demonstrations of potential of liposomes for improving ARV drug delivery, stability and leakiness of loaded drug during storage remain the issue to be sorted out (Semete et al., 2010). Additionally, low drug entrapment ability, especially for water-soluble drugs due to tiny space of aqueous core (Torchilin, 2005), is an area for improvement in liposome-based drug delivery.

\subsubsection{Solid lipid nanoparticles (SLN) based nanovehicles:}

Recently, SLN (Figure 2F) has emerged as novel particulate system with tremendous potential to be used as a drug delivery nanocarrier. For the synthesis of SLN, one or more biocompatible solid lipids such as fatty acids, glycerides, waxes, glycerine mixtures, etc. are liquefied by heating and dispersed and stabilized in either ionic or non-ionic surfactant which can be emulsifiers and/or co-emulsifiers (Gupta \& Jain, 2010; Carvalho et al., 2011). 
The size of resulting solid lipid particles may vary from 1 to $1000 \mathrm{~nm}$ and are compatible for carrying both hydrophilic and lipophilic drugs (Gupta and Jain, 2010). Because of the lesser non-specific cell toxicity, superior physical and biological stability, high tolerability, higher drug entrapment efficacy, and costeffective manufacturization, the SLN is believed to be a better nano-drug carrier than other colloidal carriers such as liposomes, PLGA, etc. (Muller et al., 1997; Mehnert and Mader, 2001; Reddy et al., 2006; Wong et al., 2010). Additionally, flexibility to modify its size and charge can be employed for the site-specific targeting and for drug release in response to specific stimuli such as temperature, $\mathrm{pH}$, etc (Bummer, 2004; Manjunath et al., 2005). Also, the immediate burst drug release profile of conventional SLN (attributable to their larger surface area) can be modified for prolonged drug release (Semete et al., 2010). All these properties in conjugation with the natural ability of small lipophilic material to cross the BBB make SLN a favorable nanocarrier for the CNS drug delivery. In this context, few studies have shown the potential applicability of SLN for ARV drug delivery across BBB. Kuo and Su (2007) used in vitro BBB model of human BMECs and demonstrated that the permeability coefficient of stavudine, delaviridine and saquinavir loaded on SLN was respectively $4-5,8-11$ and $9-11$ times as compared with free drugs. In the same study, delaviridine and saquinavir loaded on SLN showed enhanced permeability than those loaded on MMSPM; however, it was suggested that the particle size of these nanoparticles may have significant influence on their drug -loading, -entrapment and BBB permeability efficacy. Further, the same group 
(Kuo and Kuo, 2008) performed that under the influence of $5 \mathrm{mV}$ electromagnetic force (EMF) the in vitro BBB permeability of SLN loaded saquinavir was better than that loaded on PBCA and MMSPM; thus, a combination therapy, involving SLN with EMF, was recommended for the beneficial clinical application. Other in vitro BBB model study by Chattopadhyay et al (2008) showed a significantly improved cellular uptake of SLN loaded atazanavir in compare to aqueous solution. Similarly, higher cellular accumulation of Rhodamine-123, a substrate of efflux transporter P-gp, was also shown in this study. Thus, it was predicted that SLN may either mask or bypass the efflux pump. Despite these early promising in vitro data, supportive in vivo experiments are yet to be tested. Thus, more in vitro and in vivo study are necessary to delineate the authenticity of SLN for the delivery of ARV drugs in brain.

\subsubsection{Magnetic nanovehicles:}

Magnetite $\left(\mathrm{Fe}_{3} \mathrm{O}_{4}\right)$ and maghemite $\left(\mathrm{Y}-\mathrm{Fe}_{2} \mathrm{O}_{3}\right)$ are the most commonly used magnetic nanoparticles (MNPs) in the field of biomedicine (Chomoucka et al., 2010; Yoo et al., 2012). They have been extensively investigated for targetspecific improved drug delivery. The main advantage that makes MNP superior over other counterparts such as liposomes, micelles, polymeric nanoparticles, etc. is that the unique superparamagnetism property can be utilized for simultaneous monitoring and quantitation of their tissue-specific or nonspecific

distribution (Jain et al., 2008). Thus, techniques like magnetic resonance 
imaging (MRI) and magnetometery can be applied for, though indirect, measurement of localization of MNPs associated drugs (Koning et al., 2007) which may help in determining site-specific optimal or suboptimal dosing. Besides, MNPs possess many characteristics essential for a suitable drug delivery nanocarrier. First, synthesis of MNPs is quite easy (Wiogo et al., 2012) and it is feasible to produce monodispersed particles at the laboratory. Second, the flexibility in the size of MNPs, ranging from a few up to tens of nanometers, gives opportunity for optimization of sizes as per requirement of the study. It should be noted that the higher surface to volume ratio enhances target-affinity of MNPs in comparison to the micro-sized magnetic particles and can even manipulate and target at the subcellular organelles levels. Third, MNPs can respond to an external magnetic field. Thus, it is possible to "remote control" the movement of drug loaded nanoparticles for target-specific delivery by applying the magnetic force at the exterior of desired site (Pan et al., 2012). Fourth, as mentioned above, the MNPs can function as contrast agent for MRI because signal of protons, an essential requirement for MRI, in the periphery of particles is enhanced by the magnetic moment (Chaughule et al., 2012). Fifth, aqueous solutions of MNPs such as $\mathrm{Fe}_{3} \mathrm{O}_{4}$ perform amphoterism and develop positive or negative charges at the magnetite-water interface in $\mathrm{pH}$-dependent manner (Tombacz et al., 2006;). The flexibility in the surface charge allow binding of wide range of molecules either via direct, but week, ionic interactions to the MNPs (Saiyed et al., 2009) or via surface coating or tethering agents (Yoo et al., 2012; Amal et al., 2012). The well-defined and rigid structures of MNPs, with 
or without coating, further widen the attachment options by acting as a solid binding platform for various ligands (Pan et al., 2012). Sixth, in combination with the liposomes, MNPs can also be developed as hybrid nanoparticles called “magnetoliposomes" (Figure 3).

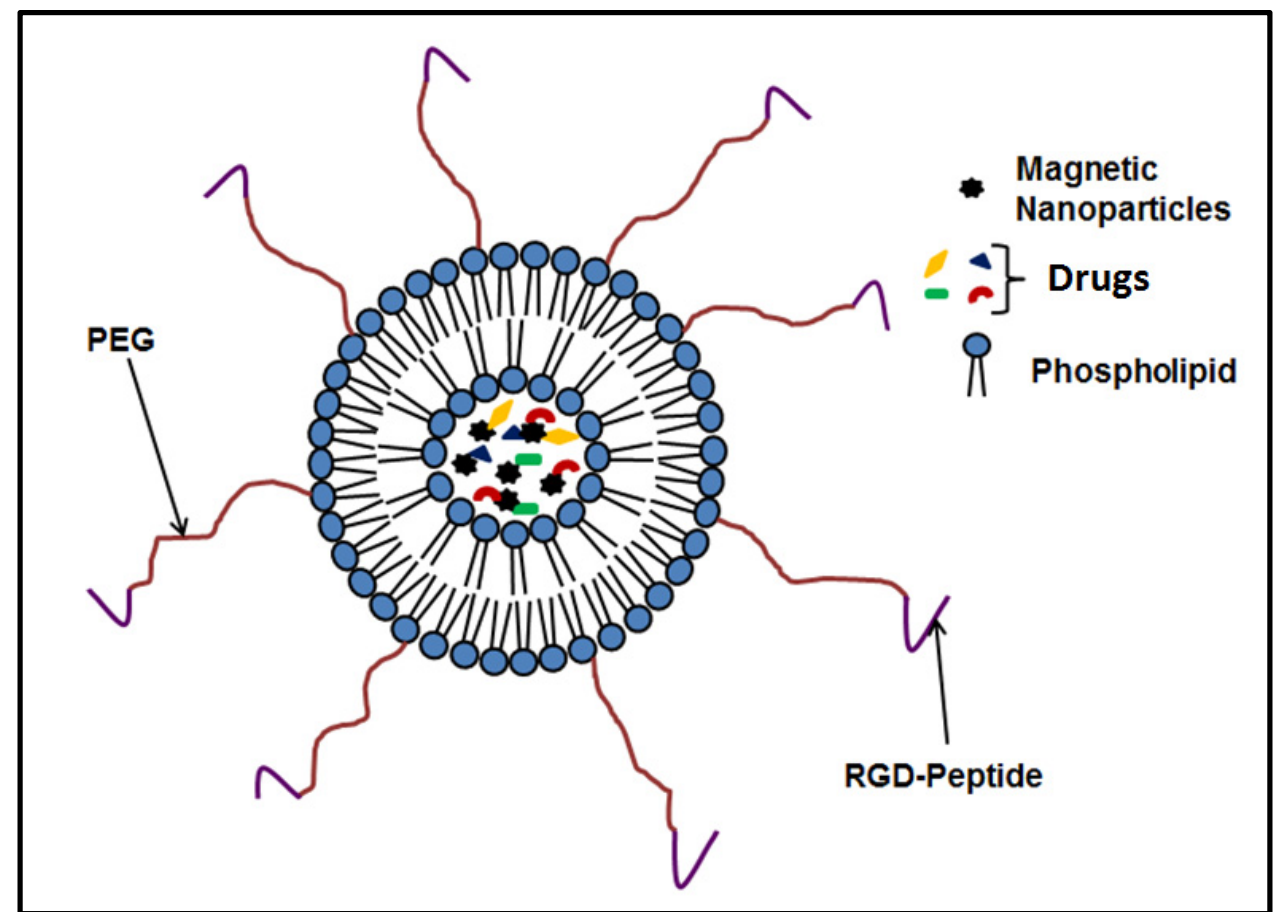

Figure 3: Magnetic Nanoparticles based nanovehicles: Magnetoliposome for drug delivery across BBB.

The liposomal encapsulation of MNP is advantageous in many ways. While drugs attached with MNPs can be encapsulated in the liposomal core, additional free drugs can be supplemented on the phospholipid bilayers and core as well. Thus, per unit loading efficiency of nanocarrier is enhanced. Also, the liposomal encapsulation protects the drug loaded on the MNPs from the biological degradations and increase the circulation time resulting in increased bioavailability. Furthermore, magnetoliposomes can be utilized for the 
monocytes/macrophage-based nanodrug delivery at the various inflammatory sites including the brain (Jain et al., 2003; Saiyed et al., 2010). The movement of magnetoliposomes or magnetized monocytes/macrophages for targeted drug delivery can be manipulated in the same way as for naked MNPs. Seventh, doses of MNPs within the permissible limit have non-significant safety concerns and are biodegradable (Jain et al., 2008). Particularly, it has been suggested that biologically produced nanosized magnetosomes from magnetotactic bacteria, which is predicted to be highly biocompatible, can be utilized in the same way as artificially synthesized MNPs (Lefevre et al., 2011; Sun et al., 2011). Thus, we see that MNPs possess many features required to be molded for nano-drug delivery in target-specific manner.

\subsubsection{Cell-based nanovehicles:}

The inherent migratory potential of inflammatory-response cells (monocytes, macrophages, dendritic cells, neutrophils, lymphocytes, neuronal stem cells, bone-marrow derived mesenchymal stromal cells, etc) towards the zone of inflammation can be exploited for the targeted drug delivery (Batrakova et al., 2011). Although still at the preliminary stage, this relatively newly hypothesized drug delivery strategy own superior therapeutic and diagnostic potential. While cells can be genetically modified for a continuous production of therapeutic molecules (Studeny et al., 2002; Muller et al., 2006; Menon et al., 2009), in the context of nanomedicine, drug loaded nanocarriers such as liposomes, magnetoliposomes, polymers, etc are either packaged inside the 
cell or, in extreme case, attached to the cell surface for the delivery at the specific injury site (Behr et al., 1997; Krantz et al., 1997; Thiele et al., 2003; Nowacek et al., 2009; Cheng et al., 2010; Zhao et al., 2011; Nair and Saiyed, 2011; Roy et al., 2011). Entry of drug loaded nanovehicles in these cells is mediated by cell surface receptors such as mannose, complement, Fc receptors, etc. Thus, coating of nanocarriers with the receptor-specific moieties such as mannose, folate, gelatin, A- protein, RGD peptide, etc complement the recognition by specific cell surface receptors leading to cellular internalization (Batrakova et al., 2011). Factors such as surface charge, size, and shape of nanocarriers also plays vital role in their internalization by cells. For example, it has been demonstrated that absorption of positively-charged nanoparticles by cells is better than their opposite counterparts (Nowacek et al., 2009). At the same time, the drug preservation efficiency of positively-charged nanoparticles in the cell is also superior (Thiele et al., 2003; Zhao et al., 2011). Once inside the cell, it is critical to home the drug-loaded cell-carriers at the right site. To this end, monocytes and macrophages have gained considerable attention for delivery of drug across the CNS (Dou et al., 2006; 2009; Muthana et al., 2008). These immunocytes possess margination and extravasation properties and can cross the BBB paracellularly in response to brain inflammation (Pawlowskiet al., 1988; Lossinsky et al., 2004). Thus, "Trojan nanocarriers" residing inside these cells can be delivered in the brain (Figure 4). Uploading of drugs from cellular carriers at the delivered site is an area which is less understood and need to be intensively investigated for advancement of this novel delivery system. In this 
context, it is believed that feasibility of controlled drug release from cellular carriers may significantly rely on prolonged stay of cell-carriers at the target site, pathology-specific response (change in temperature, $\mathrm{pH}$, etc), exocytosis of drug containing intracellular vesicles, and intracellular $\mathrm{Ca}^{2+}$ concentration of carrier cells (Sollner et al., 1993; Batrakova et al., 2007; 2011). Additionally, external stimulus such as mild hyperthermia may also affect the drug uploading from cell-carriers as has been shown for anti-cancer therapy (Ikehara et al., 2006). Other area which must be addressed for better practicality of cellmediated nanocarriers is the minimization of possible cytotoxicity. Mononuclear phagocytes recruited in response to inflammatory cytokines produce reactive oxygen species. In the same line, inhibition of recruitment of monocytes/macrophages in the zone of inflammation is part of therapeutic strategies for many neurodegenerative disorders (Hendriks et al., 2005). Nonetheless, few reported studies to date indicate no shed of cytotoxicity in macrophage-mediated drug delivery in the brain (Brynskikh et al., 2010). Eventually, successful clinical application of this method will depend upon loading of nanoformulated drugs either to harvested mononuclear phagocytes from peripheral blood or to artificially differentiated monocytes from harvested stem cells from bone marrow and their re-infusion/infusion to patients (Batrakova et al., 2011). Injection of nanoformulations, coated with monocytes/macrophages specific receptors, in blood circulation may also be another way for development of cell based delivery in clinical settings 
(Bestman-Smith et al, 2000). However, sufficient research-homework is required before practical application of these speculations.

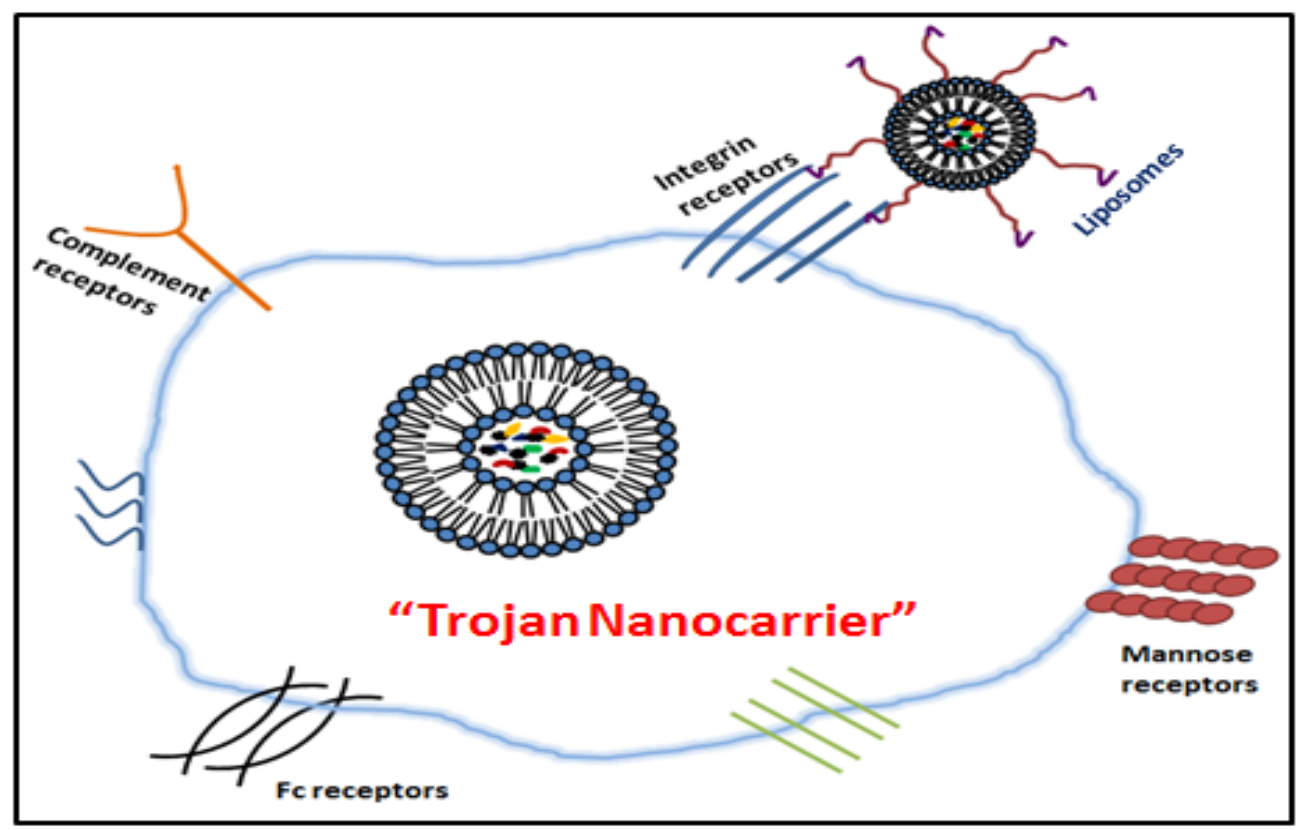

Figure 4: Cell-based drug delivery: Monocytes/Macrophages loaded with liposomal nanocarriers.

Cell mediated delivery of nanoformulated drugs is gaining significant consideration for the treatment of various brain diseases, specifically in chronic pathologies such as Alzheimer's, Parkinson's, brain cancer, epilepsy, etc (Popescu et al., 2006; Batrakova et al., 2007; Boison et al., 2009; Garcia et al., 2010; ). Its implications in HIV related neuropathogenesis has also shown encouraging trends. Dou et al., (2009) demonstrated that macrophage-based nanoparticle platform can successfully deliver the active ARV drug in the brain. Indinavir formulated in suspensions of lipid nanocrystals were packaged into ex vivo cultivated bone-marrow-derived macrophages and injected intravenously into severely combined immunodeficient HIV-1 encephalitis (HIVE) mice. High 
drug release in different regions of the brain were noticed consistently for at least two weeks and corresponding reduction in HIV replication were observed in the HIVE brain regions. In the same line, Nowacek et al., (2010) nanoformulated a combination of atazanavir, efavirenz, and ritonavir in a mixture of block copolymers and liposomes and loaded into MDM. In vitro drug release study demonstrated their continuous presence from minimum of 15 days to more than 20 days with complete suppression of viral infection. Basic of this study was recently expanded with another nanoformulated combination of atazanavir, ritonavir, indinavir, and efavirenz. By co-cultivating the drugspacked mononuclear phagocytes with human brain microvascular endothelial cells, it was suggested that intracellular crosstalk may facilitate transfer of drugs from carrier/donor cells to recipient cells (Kanmogne et al., 2012). However, mechanisms of this cell-to-cell transfer have not been explained.

\subsubsection{Other promising nanovehicles for ARV drug delivery across BBB:}

The growing popularity of nanotechnology in recent years has steadily opened the field for variety of nanomaterials. With the same perspective, many nanocarriers have been explored for their possible application in the field of nanomedicine. Two novel polymeric materials, nanoemulsions and lipid nanocapsules ( $\mathrm{LNC}$ ) (Figure $2 \mathrm{G}$ and $\mathrm{H}$ ), have been preliminarily investigated for their ability to deliver ARV drugs in brain. Vyas et al., (2008) demonstrated that the oral administration of saquinavir loaded oil-in-water nanoemulsions to Balb/c mice improved the brain uptake in compare to the aqueous formulation. 
It was suggested that higher availability of drug in the brain may be result of the higher rate of absorption of drug encapsulated in nanoemulsions. Potential of other polymeric novel nanoparticles, LNC, for the delivery of ARV protease inhibitor (Indinavir) in the mouse brain was evaluated by Pereira et al., (2005). It was found that tissue/plasma ratios of LNC loaded Indinavir in the brain of normal $\left(\mathrm{mdr} \mathrm{a}^{+/+}\right)$or efflux transporter, P-gp deficient $\left(\mathrm{mdr} 1 \mathrm{a}^{-/-}\right)$mouse increased by 1.9 times on average as compared with Indinavir in aqueous solution. At the same time, ratio of aqueous Indinavir in the brain of mdr1a ${ }^{-/-}$ mouse was 21.3-fold higher than mdr1 $\mathrm{a}^{+/+}$mice suggesting that mechanisms other than, or additional to, P-gp inhibition may influence the higher uptake of LNC loaded drugs.

Few other nanocarriers such as gold nanoparticles (Bowman et al, 2008; Reynolds et al, 2012a; 2012b; Arnaiz et al, 2012), silver nanoparticles (Elechiguerra et al, 2005; Lara et al, 2010 \& 2011), aptamers (Neff et al, 2011; Zhou and Rossi, 2011; et al, 2011; Ramalingam et al, 2011), carbon nanotubes (Liu et al, 2007; Cheng et al, 2010), quantum dots (Bonoiu et al, 2009), etc. have also been explored either for their modulated antiretroviral activity or for the targeted drug delivery in the field of HIV research. However, these initial applications are either restricted to different HIV10-infected cell types or their applicability in ARV drug delivery across CNS have been derived from other brain related, but directly or indirectly connected to HIV research such as drug of abuse, integrity of $\mathrm{BBB}$, etc. Hence, further in vitro and/or in vivo 
investigations can shed light on the legitimacy of their application for ARV drug delivery in CNS.

\subsection{Nanovehicles mediated delivery of anti-abuse drugs for treatment of neuroAIDS:}

Drug addicted AIDS patients account for one-tenth of HIV-infected individuals. Addiction of abusive drugs remarkably affects the initiation of HIV infections and expedites the progression of associated pathogenesis. Particularly, neuroimmunological changes as a consequence of alterations in reward or relapse pathways associated with drug abuse (Coller and Hutchinson, 2012) significantly enhance the progression of neuropathogenesis during HIV infections (Nath et al., 2002; Burdo et al., 2006; Hauser et al., 2007; Ferris et al., 2008; Rogers, 2011). Towards this end, last two decades of systematic research on molecular mechanism for addiction therapy emphasize on use of antagonists against different neuronal and non-neuronal receptors involved in the signaling cascades induced by drugs of abuse such as dopamine receptor antagonist, opioid-antagonist, etc. (Xi et al, 2006; Shippenberg, 2009; Gerra et al, 2009; Peng et al, 2010; Karila et al, 2011; Zhu et al, 2011; Bonoiu et al, 2009; Coller and Hutchinson, 2012). Nevertheless, majority of drug formulations tested so far in the pre-clinical or clinical experimental settings for treatment of neuroAIDS is restricted to the ARV drugs only. Antiretroviral (ARV) drugs are meant to target HIV replication and have little or no effect on the HIV-associated neuronal disorder. Thus, 
supplementation of anti-dependence agents with ARV drugs in the treatment regimen of drug addicted individuals at very early post-diagnosis of HIV infection may countercheck the rate of concerted neurotoxicity and disease progression by attenuating the rewarding effects of drug abuse.

The application of nanocarriers for target-specific drug delivery may be extended to all sorts of drugs and diseases. This approach has just commenced for addiction therapy (Bonoiu et al, 2009) and attenuation of concomitant deleterious effects of drug abuse and HIV infection. Only recently, Reynolds et al (2012) reported application of gold nanoparticles mediated delivery of siRNA against galectin-1 (an adhesion molecule) in methamphetamine treated, HIV infected MDM. They showed that stimulatory effect of methamphetamine on gelatin-1 gene expression is countered due to siRNA knock down and concomitantly HIV infection is attenuated. However, any similar nanocarriers based study to counter the concerted neurodegenerative effect of abusive drugs during HIV infection is completely lacking. Thus, in our views, nanocarriers based in vitro and in vivo study must be initiated to deliver various anti-dependence agents across the BBB and subsequently the legitimacy of their use for drug abuse associated neuropathogenesis during HIV infections could be delineated. Moreover, transfer of this strategy in clinical settings may be beneficial for suppression of rate of pathogenesis in drug addicted HIV patients and, in turn, it may add to achieve near-normal life expectancy for treated individuals. 


\subsection{Future perspectives:}

Reduction or elimination of HIV load from their safe sanctuaries such as brain still remains the major limitation for treatment of this pandemic. To this end, nanomedicines have shown tremendous promise and various forms of nanovehicles are in pre-clinical stage for targeted delivery of ARV drugs to the drug-impenetrable viral sanctuaries. Thus, relevant research-homework has to be elucidated more rigorously to sort out the various associated shortcomings of this novel approach in treatment of neuroAIDS. Better structural and physiological understanding of the brain barriers, selection of safe (non-toxic and biodegradable) material for the nanovehicles, development of specific brain celltypes targeting strategies, refinement of multifunctional nanocarriers, development of on-demand drug release strategies, universal formulation schemes for intramuscular, intravenous or oral delivery, and more realistic in vivo experimentations are few areas which should be given importance to enhance the feasibility of nanodrugs to treat the AIDS related neuropathogenesis. Additionally, delivery of neuron-resuscitating agents such as exogenous neurotrophins to the affected brain may improve the survival, development and function of neurons. Furthermore, special attention should be given to generation of new strain of resistant virus while using the nanodrugs treatment. Eventually, proper pharmacokinetic and pharmacodynamics studies and large scale manufacturization will shed light for successful application of nanodrugs in more realistic clinical settings, impacting the live of HIV infected patients. 


\section{Hypothesis and aims}

Practice of nanotechnology in medicine has shown exciting prospect for development of a novel drug delivery system to administer the desired therapeutic levels across the BBB. Several nanocarrier systems such as dendrimers, polymeric nanoparticles, micelles, etc have been intensively explored (Wong et al., 2010; 2012). Nevertheless, these nano drug-delivery methods have one or other major limitations that affect the bioavailability of desired amount of drugs at the targeted site. As such, more than $99 \%$ of the nanodrugs/carriers are deposited either in liver, lungs, or other lymphoid organ before they may cross BBB (Gunaseelan et al., 2010). Additionally, poorlyunderstood and ambiguous mechanistic details behind the delivery of existing nanocarriers across the BBB are prohibiting their advancement as brain-specific nanodelivery system. So, a fast and effective way of delivering and releasing the

drugs from the carrier in the brain is very much needed to eradicate HIV reservoir. More recently, the use of magnetic nanoparticles, particularly magnetite $\left(\mathrm{Fe}_{3} \mathrm{O}_{4}\right)$, has attracted significant importance in biomedical applications (Chomoucka et al., 2010; Yoo, 2012). Magnetically guided drug targeting has been successfully demonstrated in various pathological cases including carcinomas, inflammations, etc (Senyei et al., 1978; Chertok et al., 2008; Escribano et al., 2011). However, its application in the field of HIV/AIDS is limited. We explored the potential of magnetite for delivery of CTOP, BDNF and AZTTP in brain and found that functional efficacy of MNP bound drug remains comparable to the free drug. Hence, it is postulated that ARV and anti-abuse 
drugs may be directly immobilized on the surface of MNPs $\left(\mathrm{Fe}_{3} \underline{\mathrm{O}}_{4}\right)$ via ionic interaction and under the influence of external magnetic force MNPs based nanoformulations can be delivered across the blood-brain barrier (BBB) in more effective manner leading the way for treatment of HIV and associated neurotoxicity. Accordingly, following aims are proposed:

Aim 1: To characterize binding isotherm and pharmacokinetics of MNP bound anti-opioid and neuroprotective agent and ARV drugs.

Hypothesis: (a) Aqueous solutions of MNPs perform amphoterism and develop positive or negative charges at the magnetite-water interface in $\mathrm{pH}$-dependent manner. Charge on the surface of magnetic particles can allow either week or strong interaction of ARV and anti-opioid agents. (b) AZTTP is an established ARV drugs that have inadequate or zero reachability across the BBB under normal condition. Similarly, CTOP and BDNF cannot cross the BBB. (c) Drugs bound to MNPs may sustain its biological activity and efficacy may remain comparable to free drugs.

Aim 2: Development of liposome-based magnetic nanocarriers.

Hypothesis: (a) Encapsulation of MNPs within the core of liposomes will lead to formation of magnetoliposomes which could be a very promising material for clinical applications because it remarkably improves the drug stability and bioavailability in circulation. (b) Magnetoliposomes can be transported across BBB under influence of external magnetic force. (c) The hybrid form of MNPs (magnetoliposomes) can reduce the decomposition of drugs due to metabolic 
(enzymztic mainly) activity of peripheral circulation (blood), and clearance and entrapment by reticuloendothelial systems.

Aim 3: To evaluate the transmigration efficacy of MNP with or without drugs across the in vitro BBB model and determination BBB integrity.

Hypothesis: (a) MNPs guided nanocarriers may offer potential lead for delivery of ARV drugs across BBB by application of an external magnetic force which, in turn, can inhibit viral replication in the CNS. Similarly, CTOP and BDNF should antagonize the neurotoxic effect of morphine (b) CNS specific homeostasis such as change in temperature, $\mathrm{pH}$, etc. may trigger the drug release from MNPs. (c) Due to non-invasive nature of magnetic force, integrity of BBB will remain intact. 


\section{Materials and Experimental approaches}

\subsection{Materials:}

Iron (III) chloride $\left(\mathrm{FeCl}_{3}\right.$, Cat\#: 451649), sodium sulfite $\left(\mathrm{Na}_{2} \mathrm{SO}_{3}\right.$, Cat\#: 71989), hydrochloric acid ( $\mathrm{HCl}, \mathrm{Cat \# :} \mathrm{320331),} \mathrm{Ammonium} \mathrm{thiocyanate}$ $\left(\mathrm{NH}_{4} \mathrm{SCN}\right.$, Cat\#: 431354), ammonium persulfate $\left(\left(\mathrm{NH}_{4}\right)_{2} \mathrm{~S}_{2} \mathrm{O}_{8}\right.$, Cat\#: 248614), ammonium hydroxide solution $\left(\mathrm{NH}_{3} . \mathrm{H}_{2} \mathrm{O}\right.$, Cat\#: 320145), and Transferrin (Cat\#: T4132) were purchased from Sigma-Aldrich (St. Louis, MO, USA). Unless notified, water used for all reactions was HPLC grade. Egg phosphatidylcholine (EPC, Cat\#: 830071P), 1,2-distearoyl-sn-glycero-3-phosphoethnol- amine-N[methyoxy(polyethylene glycol)-2000] (mPEG $2000-\mathrm{DSPE}$, Cat\#: 880128P), 1,2distearoyl-sn-glycero-3-phosphoethnolamine-N-carboxylfluorescein (CFPE, Cat\#: 810332), and cholesterol (Chol, Cat\#: 700000P) were obtained from Avanti Polar Lipids (Alabaster, AL, USA). The tetrazolium compound [3-(4, 5-dimethyl thiazol2-yl)-5-(3-carboxy-methoxyphenyl)-2-(4-sulfophenyl)-2H-tetrazolium, inner salt (MTS, Cat\#: G3580) was purchased from Promega Corporation (Fitchburg, WI, USA). Human brain endothelial cell (HMBVEC, Cat\#: 1000), human brain astrocytes (HA, Cat\#: 1800) and their culture medium (Cat\#: 1001 and 1801 respectively) were brought from ScienCell Research Laboratories (Carlsbad, CA, USA).

\subsection{Synthesis of magnetic nanoparticles:}

Magnetic nanoparticles were prepared by co-precipitating of $\mathrm{Fe}^{2+}$ and $\mathrm{Fe}^{3+}$ ions in alkaline solution and treating under hydrothermal condition as described 
earlier (Saiyed et al., 2007). Hundred millilitres solution of $1 \mathrm{M} \mathrm{FeSO}_{4} \cdot 7 \mathrm{H}_{2} \mathrm{O}$ and $2 \mathrm{M} \mathrm{FeCl}{ }_{3}$ (Sigma) were thoroughly mixed and added to $8 \mathrm{M}$ ammonium hydroxide (Sigma) with constant stirring at $25^{\circ} \mathrm{C}$. The resultant black magnetite particles were washed repeatedly with hot distilled water to remove impurity ions such as chlorides and sulphates and dispersed in Tris-EDTA buffer ( $\mathrm{pH}$ 7.5). The yield of precipitated magnetic nanoparticles was determined by removing known aliquots of the suspension and drying to a constant mass in an oven at $60{ }^{\circ} \mathrm{C}$. Finally, the particles were dispersed in TE buffer at a suspension concentration of $10 \mathrm{mg} / \mathrm{ml}$. The particles were characterized for size using transmission electron microscopy (TEM).

\subsection{Characterization of MNPs:}

\subsubsection{X-ray diffraction (XRD) and transmission electron microscopy (TEM)} analysis:

Structural conformation of MNPs was determined by Shimadzu XRD-7000 diffractometer (Shimazdu, Tokyo, Japan). TEM of MNPs was performed with the Phillips CM-200 $200 \mathrm{kV}$ transmission electron microscope operated at $80 \mathrm{kV}$. A drop of MNPs was spread on carbon support film on 400 mesh Cu grids (Type B, Ted Pella, Inc., USA). For better contrast during TEM imaging, samples on grid were negatively stained with phosphotungstic acid $(2.0 \% \mathrm{w} / \mathrm{v} ; \mathrm{pH} 6.4)$ and dried at room temperature. 


\subsubsection{Particle size and zeta potential}

The hydrodynamic radius, size distribution, and surface charge measurement of MNPs were carried out at $25^{\circ} \mathrm{C}$ in dynamic laser scattering (DLS) (90 Plus Particles Size Analyzer, Brookhaven Instrument Corp., USA). Samples were prepared by diluting equal quantity of magnetic particles in different $\mathrm{pH}$ range of Tris-HCL and Tris-EDTA buffer.

\subsubsection{Superparamagnetism measurement}

The measurement of superparamagnetism was carried out by classical vibrating sample magnetometer (Model 4HF VSM, USA). The magnetic hysteresis loops of the $\mathrm{Fe}_{3} \mathrm{O}_{4}$ particles were measured between +1200 to -1200 Oersted $(\mathrm{Oe})$ at room temperature.

\subsection{Binding of drugs onto the magnetic nanoparticles:}

\subsubsection{CTOP binding to magnetic nanoparticles:}

Mixture of MNPs and CTOP procured from Sigma Aldrich at different mole ratios $(1: 0.16,1: 0.33,1: 0.66,1: 1$, and 1:1.33) were incubated in tube rotator at room temperature and supernatant were collected for quantification of unbound CTOP by HPLC. The difference between the total CTOP added and unbound CTOP was used to calculate the amount of CTOP bound to the MNPs.

\subsubsection{High-performance liquid chromatography/Photo diode array} (HPLC/PDA):

HPLC/PDA analyses were performed with a P4000 Thermo-Finnigan chromatograph (Thermo Electron Corporation, San Jose, California) and 
consisted of a SpectraSystem SMC1000 solvent delivery system, vacuum membrane degasser, P4000 gradient pumps and AS3000 autosampler. Column effluent was monitored at $254 \mathrm{~nm}$ with a SpectraSystem UV6000LP variable wavelength PDA detector and ChromQuest 4.1 software. Analytical separations were carried out with a $\mathrm{C}_{18} \mathrm{RP}$ Hypersil GOLD column (RP5, $250 \times 4.6 \mathrm{~mm}$, pore size $5 \mu \mathrm{m}$, Thermo Electron Corporation). The mobile phase consisted of $0.1 \%$ TFA in $\mathrm{MeCN}$ (phase $\mathrm{A}$ ) and $0.1 \%$ TFA in $\mathrm{H}_{2} \mathrm{O}$ (phase $\mathrm{B}$ ). The linear gradient program was as follows: 10 to $100 \% \mathrm{~A}$ over $30 \mathrm{~min}$ at a flow rate of $1.0 \mathrm{~mL} / \mathrm{min}$; 10-20 $\mu \mathrm{L}$ of solution were usually injected. Peptide: Rt, $11.30 \mathrm{~min} ; \lambda \max 276 \mathrm{~nm}$.

\subsubsection{Fourier transform infrared spectroscopy (FTIR):}

FTIR spectroscopy was performed on drug loaded and free nanoparticles to examine the immobilization of CTOP on its surface. MNPs bound CTOP isolated from binding reaction mixture were lyophilized to preserve the integrity of drug in the dried and powered MNPs colloids. These powdered samples were used for FTIR analysis in the IR spectrophotometer (Perkin Elmer Spectrum $^{\mathrm{TM}}$ 100). Spectra measurement were performed using attenuated total reflection (ATR) on a single bounce diamond/ZnSe ATR crystal. The spectra were collected in the mid-infra red range of $4000-600 \mathrm{~cm}^{-1}(2.5-25 \mu \mathrm{m})$.

\subsubsection{Fluorescent tagging of CTOP for binding validation:}

Alexa flour 610 succinimidyl ester (NHS ester) (Life Technologies) were used for tagging of CTOP. NHS esters were mixed with equal amount of CTOP or PBS and were incubated overnight at room temperature. MNPs were added in the mixture and rotate in tube rotator at room temperature. The collected 
magnetic particle is used for quantification of attached peptide. Fluorescent intensity was measured at wavelength 485/20nm-528/20 nm (Ex/Em) by microplate reader (Synergy HT, Multi-mode microplate reader, BioTek Instrument, Inc., Winooski, Vermont, USA). MNPs added in mixture of NHS esters and PBS does not show any fluorescent.

\subsubsection{BDNF binding with magnetic nanoparticles:}

For the binding experiment, different ratios of magnetic nanoparticles and $\operatorname{BDNF}(1: 0.05,1: 0.01,1: 0.015,1: 0.02,1: 0.025,1: 0.03$, and 1:0.35) were mixed in TE buffer $\mathrm{pH} 7.4$, followed by incubating the mixture on a shaker (100 rpm) for 3 hrs at room temperature. After incubation, the magnetic particles bound with BDNF were attracted by application of an external magnetic field. The supernatant containing the unbound BDNF was collected and the pellet was resuspended in appropriate volume of TE buffer $\mathrm{pH} 7.5$ and stored at $2^{\circ} \mathrm{C}$ to $8^{\circ} \mathrm{C}$ until further use.

\subsubsection{BDNF enzyme linked immunosorbent assay (ELISA):}

The binding efficiency ( $\mu \mathrm{g}$ BDNF /mg of magnetic nanoparticles) was determined by measuring the amount of BDNF in the unbound fraction by ELISA (BDNF Kit from R\&D Systems, Minneapolis, MN, USA) as per manufacturer's recommendation. $100 \mu \mathrm{l}$ of assay diluent were added to each well of microplate and were mixed with $50 \mu \mathrm{l}$ standard, control, or sample. Plates were covered with a plate sealer and incubated at room temperature for 2 hours. $100 \mu \mathrm{l}$ conjugate were added to each well following incubation and further incubated at room 
temperature for 1 hour. Each well was aspirated and washed with washing solution, supplied with $200 \mu \mathrm{l}$ substrate solution and incubated in dark at room temperature for 30 minutes. $50 \mu \mathrm{l}$ stop solution is added to each well and fluorescent intensity is read at $450 \mathrm{~nm}$ by microplate reader (Synergy HT, Multimode microplate reader, BioTek Instrument, Inc., Winooski, Vermont, USA). The amount of BDNF bound to the magnetic nanoparticles was calculated from the difference between the total BDNF added and unbound BDNF measured in the supernatant.

\subsubsection{Fluorescent tagging of BDNF for binding validation:}

Alexa flour 610 succinimidyl ester (NHS ester) (Life Technologies) were used for tagging of CTOP. NHS esters were mixed with equal amount of BDNF or PBS and were incubated overnight at room temperature. MNPs were added in the mixture and rotate in tube rotator at room temperature. The collected magnetic particle is used for quantification of attached peptide. Fluorescent intensity was measured at wavelength 485/20nm-528/20 nm (Ex/Em) by microplate reader (Synergy HT, Multi-mode microplate reader, BioTek Instrument, Inc., Winooski, Vermont, USA). MNPs added in mixture of NHS esters and PBS does not show any fluorescent.

\subsection{Cell Culture}

\subsubsection{Preparation of Peripheral blood mononuclear cells (PBMC)}

Normal peripheral blood mononuclear cells were isolated by density gradient centrifugation process as described by Gandhi et al (2009). The 
Buffycoat leukopack, procured from the community blood bank, was diluted by adding five volumes of phosphate-buffered saline (PBS) and overlaid over histopaque (Sigma Aldrich, St. Louis, MO). The samples were centrifuged at $1200 \times g$ for $20 \mathrm{~min}$ at room temperature. PBMCs were carefully retrieved from the interface and washed twice with PBS. Red blood cells in the samples were lysed using Ammonium-Chloride-Potassium Lysing Buffer (Life Technologies). Collected cell pellet were resuspended in Roswell Park Memorial Institute Medium (RPMI) -1640.

\subsubsection{Peripheral blood mononuclear cells (PBMC) culture:}

PBMCs isolated by density gradient centrifugation were cultured in RPMI 1640. RPMI 1640 culture medium was supplemented with $10 \%$ fetal bovine serum (FBS), $100 \mathrm{U} / \mathrm{ml}$ penicillin, and $100 \mathrm{mg} / \mathrm{ml}$ streptomycin (Gibco-BRL, Gaithersburg, MD). Cells were cultured at $37^{\circ} \mathrm{C}$ in $5 \% \mathrm{CO}_{2}$ incubator.

\subsubsection{SK-N-MC cell culture:}

SK-N-MCs, a neuroepithelioma cell line derived from a metastatic supraorbital human brain tumor, were cultured in minimum essential medium (MEM). MEM was supplemented with $10 \%$ fetal bovine serum (FBS), $100 \mathrm{U} / \mathrm{ml}$ penicillin, and $100 \mathrm{mg} / \mathrm{ml}$ streptomycin (Gibco-BRL, Gaithersburg, MD). Cells were incubated at $37^{\circ} \mathrm{C}$ in $5 \% \mathrm{CO}_{2}$ incubator.

\subsubsection{Primary human astrocytes (HA) culture:}

Human astrocytes were cultured in astrocyte medium (AM) (ScienCell). AM were supplemented with $2 \%$ FBS and $1 \%$ each of astrocyte growth 
supplement and penicillin/streptomycin solution (ScienCell). Culture was performed in $37^{\circ} \mathrm{C}$ incubator with an atmosphere of $5 \% \mathrm{CO}_{2}$ and $95 \%$ air.

\subsubsection{Human brain endothelial cell (HMBVEC) culture:}

Human brain endothelial cells (HMBVEC) were brought from ScienCell Research Laboratories (Carlsbad, CA, USA) and cultured in endothelial cell medium (ECM) (ScienCell). ECM were supplemented with 5\% FBS and 1\% each of endothelial cell growth supplement and penicillin/streptomycin solution (ScienCell). Culture was performed in $37^{\circ} \mathrm{C}$ incubator with an atmosphere of $5 \%$ $\mathrm{CO}_{2}$ and $95 \%$ air.

\subsection{Efficiency of MNPs bound CTOP:}

\subsubsection{Apoptosis inhibition efficiency:}

$1 \times 10^{6}$ PBMCs were treated either with $1.5 \mu \mathrm{m}$ free or MNPs bound CTOP for 4-6 hrs before treatment of morphine $(1 \mu \mathrm{m})$. A schematic of different treatment groups are depicted in figure 5. Following $60 \mathrm{hrs}$ of morphine treatment, cells were washed twice with cold PBS and resuspended in 1x binding buffer at a concentration of $1 \times 10^{6} \mathrm{cells} / \mathrm{ml} .100 \mu \mathrm{l}$ of this resuspended cells were aliquoted into a $5 \mathrm{ml}$ FACS tubes, mixed with $5 \mu \mathrm{l}$ each of Annexin $\mathrm{V}$ and 7-AAD (BD Biosciences) and incubated at RT for 15 minutes in the dark. After incubation, $400 \mu \mathrm{l}$ of $1 \mathrm{X}$ binding buffer is added to each tube, mixed gently, and analyzed within $1 \mathrm{hr}$ by flowcytometry (FACScalibur). The untreated cells, which served as control, are used for defining the basal level of apoptotic and dead cells. The percentage of cells that have been induced to undergo apoptosis is 
then determined by subtracting the percentage of apoptotic cells in the untreated population from percentage of apoptotic cells in the treated population. Cells treated with camptothecin for $5 \mathrm{hrs}$ at $37^{\circ} \mathrm{C}$ is used as positive control.

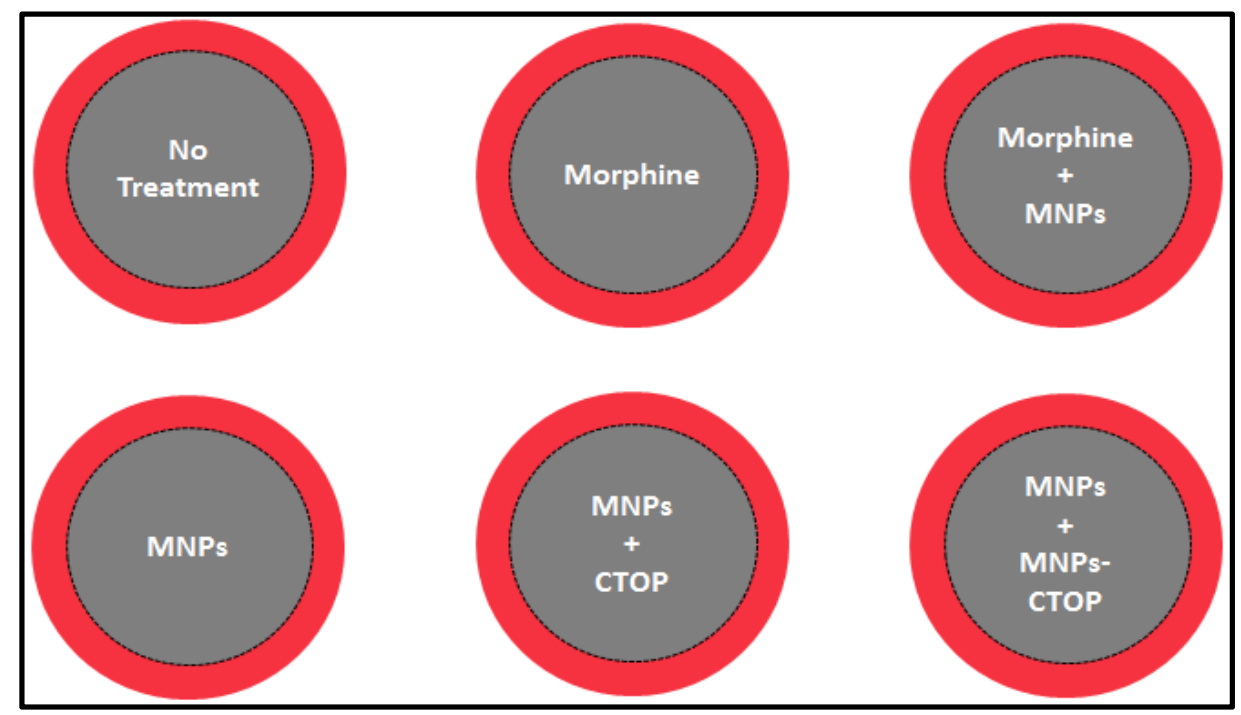

Figure 5: Schematic of the experimental design applied for apoptosis inhibition assay with MNPsCTOP nanoformulations.

\subsubsection{Characterization of neuro-spinal architecture:}

\subsubsection{SK-N-MC staining:}

Membrane staining of neuronal cells and measurement of spine density was performed according to the method adopted from Smith et al (2009). $0.5 \times 10^{6}$ SK-N-MC cells were seeded onto $22 \times 50 \mathrm{~mm}$ glass coverslips placed in a petri-dish. Following $24 \mathrm{hr}$ of culture, cells were treated either with $1.5 \mu \mathrm{m}$ free or MNPs bound CTOP for 4-6 hrs before treatment of morphine $(1 \mu \mathrm{m})$. Media from the treatment was replaced every alternated day of culture and accordingly fresh or MNPs-bound CTOP were added to each treatment. Different groups for this treatment were similar to that shown in figure 5 . Cells after $7^{\text {th }}$ days of treatment 
were washed with PBS and fixed in 4\% paraformaldehyde for 30 min at room temperature. The fluorescent membrane tracer 1, 1'-Dioctadecyl-3,3,3',3'tetramethylindocarbocyanine perchlorate (DIL; $5 \mu \mathrm{g} / \mathrm{ml}$ in PBS) was directly added onto the fixed cells and allowed to incubate at room temperature for $60-90$ min. Further, coverslips are placed in small petri dishes containing PBS and incubated at $4^{\circ} \mathrm{C}$ for $12 \mathrm{hr}$ before preparation for confocal microscopy.

\subsubsection{HIV co-infection of SK-N-MC with morphine treatments:}

$0.5 \times 10^{6}$ SK-N-MC cells were seeded onto $22 \times 50 \mathrm{~mm}$ glass coverslips placed in a $90 \mathrm{~mm}$ petri-dish and allowed to adhere overnight. Cells were treated with polybrene $(10 \mu \mathrm{g} / \mathrm{ml})$ and $8 \mathrm{hrs}$ following this treatment $100 \mathrm{ng}$ clade B HIV-1 was added to each treatment. $12 \mathrm{hr}$ post-infection, non-absorbed virus was washed with PBS, and HIV infection was carried for 7 days. Infected cells were treated with Morphine and CTOP as explained above. Similarly, co-infected cells were DIL stained and prepared for confocal microscopy. A schematic of different co-infections groups are depicted in figure 6.



Figure 6: Schematic of the experimental design applied for efficiency determination of MNPsCTOP nanoformulations on morphine and HIV co-infection induced neuropathogenesis. 


\subsubsection{Confocal Microscopy:}

DIL stained SKNMC were mounted on the ProLong Gold antifade reagents (life technologies) and dried away from light at room temperature. Leica confocal laser scanning microscope was used for the morphological imaging of these cells. Images were obtained using $60 x$ oil immersion objectives at $488 \mathrm{~nm}$ (100\%) illusion of an argon-ion laser. High numeric aperture and 2.5x electronic zoom were maintained to visualize the individual cells and their spines. Three dimensional images of individual cells were obtained using optical serial sections at the rate of $0.14 \mu \mathrm{m} / \mathrm{section}$ of the cells. Obtained images were used for quantification of spinal density through ImageJ software program. Randomly chosen dendritic segments of at least 10 cells from each treatment were measured from the apical to basal regions away from the cell soma and spine numbers were counted within this defined length. As shown in figure 7 , spine density is calculated as the number of spines per unit dendritic length and expressed as spines/ $\mu \mathrm{m}$.

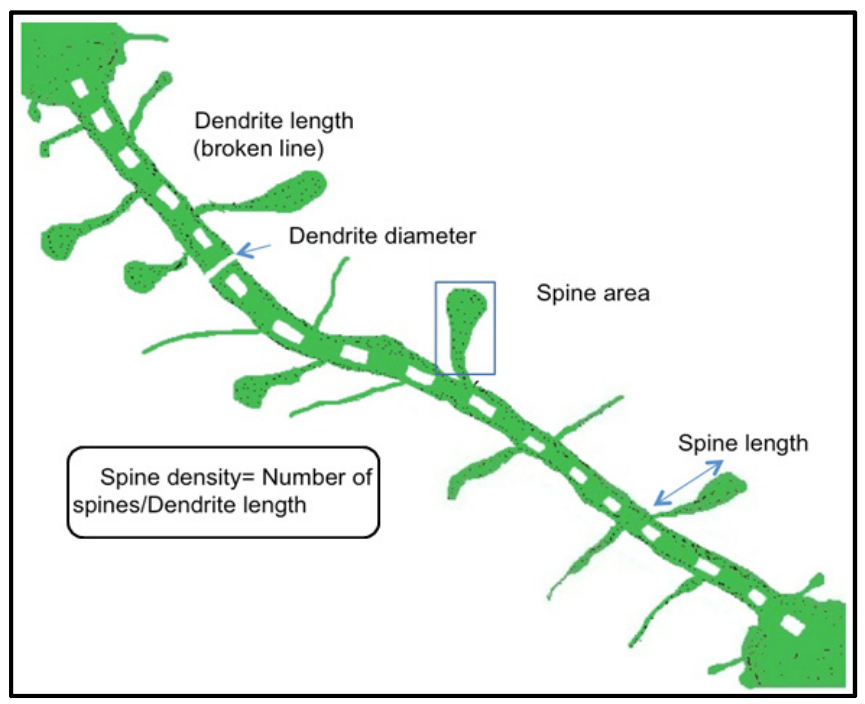

Figure 7: Schematic of a typical dendrite segment: Spine density from is measured by calculating total number of spines in dendrite segment divided by the dendrite length (Venkata et al., 2013). 


\subsubsection{Validation of HIV infection:}

The culture supernatant from HIV infected SK-N-MC cells were collected for quantitation of HIV p24 antigen using a p24 ELISA kit (ZeptoMetrix, Buffalo, NY). Supernatant from each treatment groups were thoroughly mixed with lysing buffer in 1:9 ratios. $200 \mu \mathrm{l}$ of lysed samples were added in triplicate in different wells of microplate. These wells are coated with anti-p24 monoclonal antibodies which allow binding of p24 from lysed samples at $37^{\circ} \mathrm{C}$ overnight incubation. Now, samples from each well was aspirated and washed 5-6 times with the wash buffer. $100 \mu \mathrm{l}$ of HIV-1 p24 detector antibody (biotin conjugated) was added to each well and incubated at $37^{\circ} \mathrm{C}$ for 1 hour. Again wells were washed $5-6$ times, supplied with $100 \mu \mathrm{l}$ of streptavidin-peroxidase working solution, and incubated at $37^{\circ} \mathrm{C}$ for 30 minutes. Solution from wells were aspirated, washed, and $100 \mu \mathrm{l}$ of substrate working solution is added into each well and incubated at room temperature. Upon change of color to blue, $100 \mu \mathrm{l}$ stop solution was added to each well which cause change of color into yellow. The optical density of microplate at $450 \mathrm{~nm}$ gives the quantity of HIV p24 in each well. Different concentration of standard p24 antigen is also used simultaneously to plot standard curve for quantitation.

\subsubsection{Cell viability assay:}

The MTT (Thiazolyl blue tetrazolium bromide) cell proliferation assay was performed as described by Wilson et al (1990) with modifications. $1 \times 10^{6}$ PBMCs were treated either with $1.5 \mu \mathrm{m}$ free or MNPs bound CTOP for 4-6 hrs before 
treatment of morphine $(1 \mu \mathrm{m})$. A schematic of different treatment groups are depicted in figure 8 . Following 48 hrs of morphine treatment, cells were treated with $0.5 \% \mathrm{MTT}$ at the rate of $100 \mu \mathrm{l}$ per well and gently rocked in dark at room temperature for $2-3 \mathrm{hrs}$. One volume STOP solution containing $20 \%$ SDS in $50 \%$ dimethyl formamide were added to the rocking cell suspension in MTT solution and further rocked gently in dark at room temperature for 1-2 hrs. Cell suspension is centrifuged at $2000 \mathrm{rpm}$ for 10 minutes and supernatant were collected for the spectrophotometric measurement of optical density of the solubilized formazan at $550 \mathrm{~nm}$. The optical density of formazan in each treatment groups is directly proportional to the cell viability.

Cell viability for SK-N-MC were determined similar to that for PBMCs except overnight culture media were changed with $1 \mathrm{ml}$ fresh media before addition of $0.5 \% \mathrm{MTT}$.

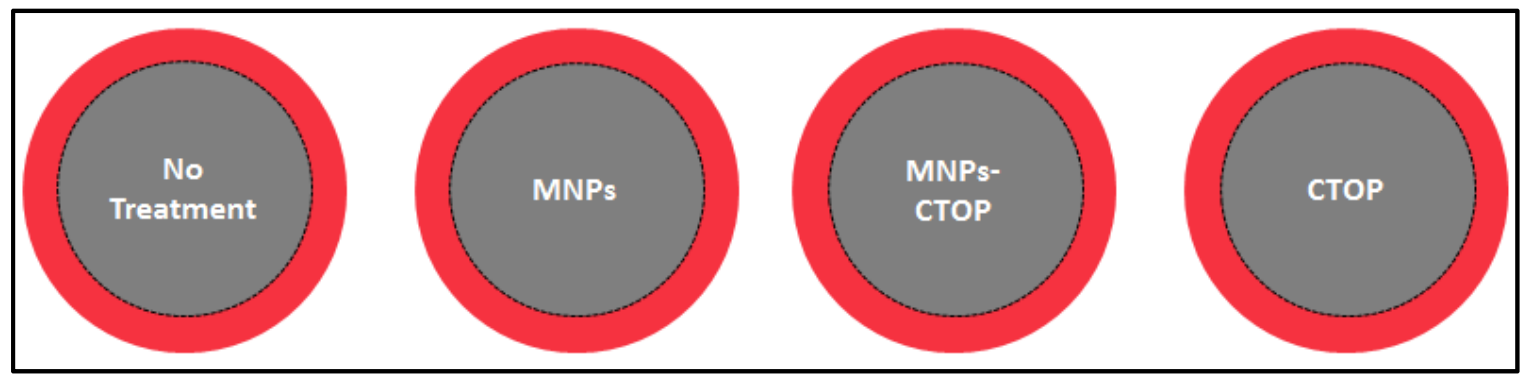

Figure 8: Schematic of the experimental design applied for cell-viability assay with MNPs-CTOP nanoformulations.

\subsection{Efficiency of MNPs bound BDNF:}

\subsubsection{Apoptosis inhibition efficiency:}

To see the protective effect of BDNF on morphine induced apoptosis, $1 \times 10^{6}$ PBMCs were treated with free and MNPs bound BDNF $12 \mathrm{hr}$ before 
morphine treatment. Different doses of BDNF used were 10,50 , and $100 \mathrm{ng} / \mathrm{ml}$. The concentration showing optimum efficacy was used for efficiency-verification of MNPs-BDNF nanoformulations. Morphine was treated with a concentration of $10^{-7} \mathrm{M}$ for $48 \mathrm{hrs}$ and cells were processed for flowcytometry (FACScalibur) based apoptosis measurement as described for the CTOP efficiency measurement. A schematic of different treatment groups are depicted in figure 9 and 10.

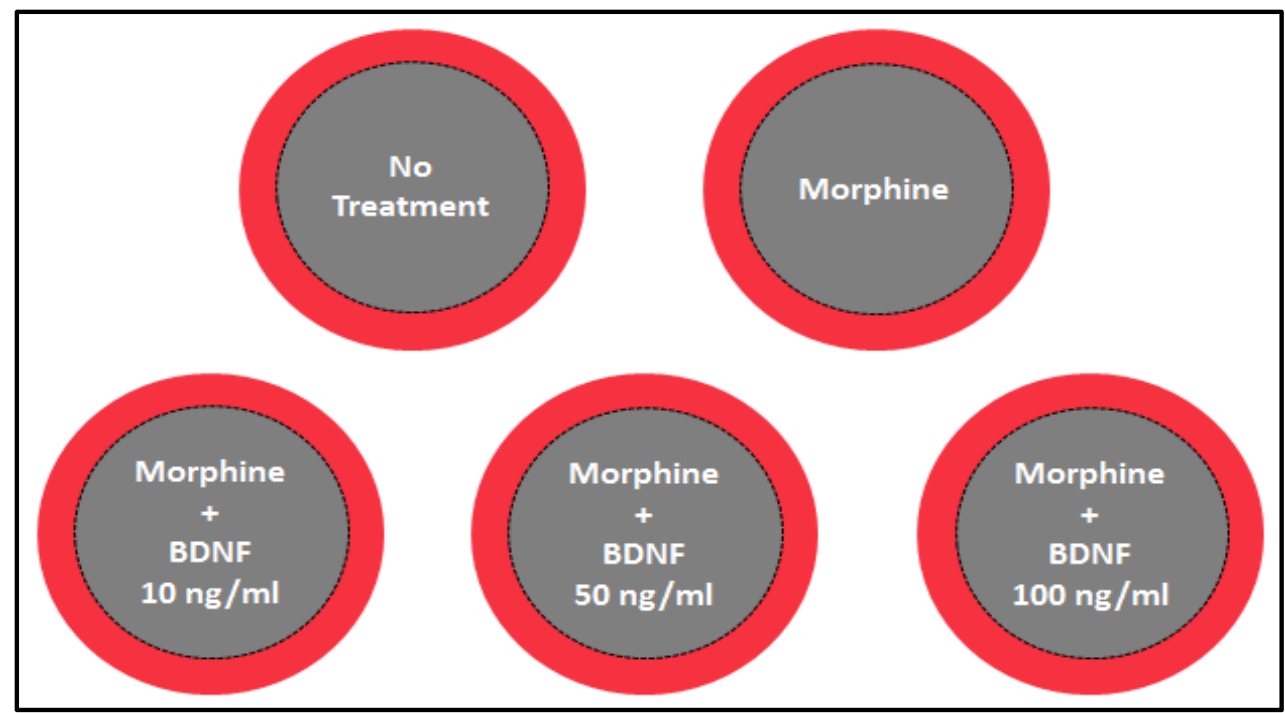

Figure 9: Schematic of the experimental design applied for determination of optimum BDNF concentration required for inhibition of morphine-induced apoptosis in PBMCs.

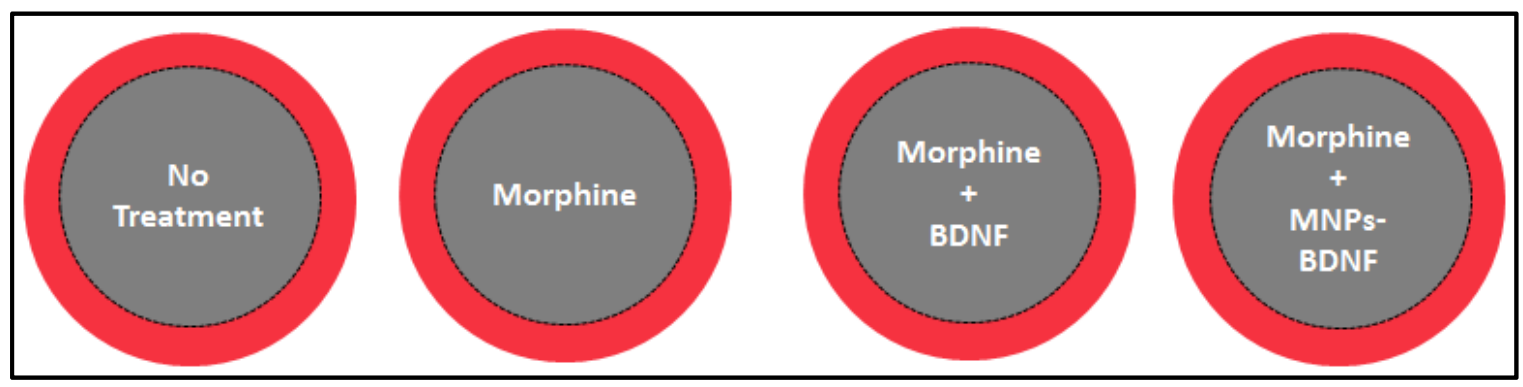

Figure 10: Schematic of the experimental design applied for apoptosis inhibition assay with MNPs-CTOP nanoformulations. 


\subsubsection{Quantification of cAMP response element-binding protein (CREB):}

\subsubsection{RNA isolation:}

To see the BDNF-mediated effect on the expression of cAMP response element-binding protein (cAMP), astrocytes were treated with free and MNPs bound BDNF in the presence and absence of Morphine. Different treatment groups for this experiment were similar that depicted in figure 10. Total RNA from each treatment groups was isolated using RNAeasy kit (Qiagen) as per the manufacturer's protocol. 5-10 $\times 10^{6}$ cells were vortex-lysed using RLT buffer supplied with the kit. Lysate were loaded onto the QIAshredder spin columns and filtered by centrifugation at 10,000 rpm for 30 seconds. Filtered flow through were mixed thoroughly with $350 \mu \mathrm{l} 70 \%$ ethyl alcohol and filtered in RNeasy silica membrane column (RNeasy spin column) by centrifugation at 10,000 rpm for 30 seconds. Bound RNA in the column is washed once with $700 \mu \mathrm{RW} 1$ buffer and twice with $500 \mu \mathrm{l}$ RPE buffer, each at $10,000 \mathrm{rpm}$ for $30 \mathrm{sec}$. Finally, RNA is washed with $50 \mu \mathrm{l}$ water for elution and stored at $-80^{\circ} \mathrm{C}$ for future use.

\subsubsection{Synthesis of cDNA:}

Isolated total RNA was used for the synthesis of cDNA using high capacity cDNA reverse transcription kits (Applied Biosystems). As per the manufacturer's protocol, 2x cDNA master mixes were prepared by mixing 10x reverse transcription buffer, 25x dNTP mix, 10x random primers, RNAse inhibitors, and reverse transcriptase enzyme. Total volume was adjusted to $20 \mu \mathrm{l}$ with DNAse and RNAse free water. Ratio of each components of this master mix is presented in table $1 \mathrm{a}$ and $\mathrm{b}$. This $2 \mathrm{x}$ cDNA master mix is adjusted to $1 \mathrm{x}$ by adding one 
volume of $4 \mu \mathrm{g}$ total RNA. The mixture of master mix and RNA was subjected for the thermocycling reaction in 2720 thermocycler (Applied Biosystems). Initially reaction was fixed at $25^{\circ} \mathrm{C}$ for 10 minutes which allows primer annealing to the RNA. The reverse transcription reaction was extended at $37^{\circ} \mathrm{C}$ for 120 minutes and finally reaction is heated at $85^{\circ} \mathrm{C}$ for 5 minutes to inactivate the enzyme. The cDNA yield is stored at 4 or $20^{\circ} \mathrm{C}$ for further use.

\begin{tabular}{|l|ll|}
\hline $\begin{array}{l}10 \text { X Reverse Transcription } \\
\text { buffer }\end{array}$ & 4.0 & $\mu \mathrm{l}$ \\
\hline 25 X dNTP mix & 1.6 & $\mu \mathrm{l}$ \\
\hline 10 X Random Primers & 4.0 & $\mu \mathrm{l}$ \\
\hline Enzyme (Reverse Transcriptase) & 2.0 & $\mu \mathrm{l}$ \\
\hline RNAse inhibitor & 2.0 & $\mu \mathrm{l}$ \\
\hline Water & 6.4 & $\mu \mathrm{l}$ \\
\hline Total: & $20.0 \mu \mathrm{l}$ \\
\hline
\end{tabular}

\begin{tabular}{|l|l|}
\hline Master mix & $20 \mu \mathrm{l}$ \\
\hline mRNA & $4 \mu \mathrm{g}\} 20 \mu \mathrm{l}$ \\
\cline { 1 - 1 } Water & - \\
\hline Total: & $40 \mu \mathrm{l}$ \\
\hline
\end{tabular}

(B)

(A)

Table 1: (A) Ratios of ingredients for preparation of 2x cDNA master mix. (B) Ratios of 2x cDNA master mix and mRNA for PCR reaction during cDNA synthesis.

\subsubsection{Quantitative polymerase chain reaction ( $q P C R)$ :}

Relative abundance of CREB mRNA was assessed using the SYBR green master mix from Stratagene (La Jolla, CA, USA). CREB specific primer was brought from life technologies. Ratio of each components of this reaction mix is presented in table 2.The real-time quantitative PCR was performed using the Stratagene 3000 instrument that detects and auto-plots the difference in fluorescence versus PCR cycle number and produces a continuous measure of 
PCR amplification. Time taken by amplification plot of each sample to reach a fluorescence threshold above background gives their threshold cycle number $\left(C_{T}\right)$ which is used to calculate relative gene expression in terms of transaccumulation index. The quantity of RNA input is controlled by measuring endogenous reference gene, $\beta$-actin. Additionally, data of RNA from treated groups were normalized to that from control untreated groups.

\begin{tabular}{|l|l|}
\hline 2 X Master mix & $12.5 \mu \mathrm{l}$ \\
\hline Reference dye & $0.375 \mu \mathrm{l}$ \\
\hline 20 X Primer/Probe & $1.25 \mu \mathrm{l}$ \\
\hline Water & $8.375 \mu \mathrm{l}$ \\
\hline cDNA & $2.5 \mu \mathrm{l}$ \\
\hline Total: & $\mathbf{2 5 . 0} \mu \mathrm{l}$ \\
\hline
\end{tabular}

Table 2: Ratio of ingredients for qPCR reactions

\subsubsection{Calculation of transcript accumulation index (TAI) or relative expression}

The $\Delta C_{\mathrm{T}}$ and $\Delta \Delta C_{\mathrm{T}}$ values were calculated for quantitation of TAl (Shively et al., 2003). The $\Delta C_{T}$ values was calculated by taking mean $C_{T}$ values of CREB mRNA and subtracting the mean $C_{T}$ values of reference mRNA i.e. $\beta$-actin from the same reverse transcription reaction.

$$
\Delta C T=C T(C R E B)-C T(\beta-\text { actin })
$$


The $\Delta \Delta C_{\mathrm{T}}$ values were calculated by subtracting the $\Delta C_{\mathrm{T}}$ of the untreated control sample from that of treatment groups from the same reverse transcription reaction.

$$
\Delta \triangle C T=\triangle C T \text { (treatment groups) }-\triangle C T \text { (untreated control) }
$$

The transcript accumulation index (TAI) which is the relative gene expression is given by,

$$
\mathrm{TAI}=2^{-\Delta \Delta C T}
$$

Thus, this TAl measurement of gene expression is relative to the reference gene and normalized to the untreated group. Importantly, PCR reactions are believed to be $100 \%$ efficient. Only values with $>95 \%$ efficiencies in each reverse transcription reaction ascertain minimization of the calculations error.

\subsubsection{Characterization of neuro-spinal architecture: SK-N-MC staining and confocal microscopy:}

Membrane staining of neuronal cells, confocal microscopy, and measurement of spine density was performed similar to that described previously for CTOP mediated effect. Different treatment groups for this experiment were similar that depicted in figure 10 . In brief, $0.5 \times 10^{6}$ SK-N-MC cells were seeded onto $22 \times 50 \mathrm{~mm}$ glass coverslips placed in a petri-dish. Following $24 \mathrm{hr}$ of culture, cells were treated either with free or MNPs bound BDNF $12 \mathrm{hrs}$ before morphine treatment and cultured were maintained for 7 days. Media from the treatment was replaced every alternated day of culture and accordingly fresh or MNPs- 
bound BDNF were added to respective treatment. Cells grown on cover slip were fixed, stained, mounted, and analyzed under confocal microscopy.

\subsubsection{Cell viability assay}

Live/Dead amine-reactive dye (ViViD; Invitrogen) was used to determine the viability of PBMCs (Pilakka-Kanthikeel et al., 2013). The cells were treated either with free or MNPs bound BDNF $12 \mathrm{hrs}$ before treatment of morphine. A schematic of different treatment groups are depicted in figure 11. Following 48-60 hrs of this treatment, PBMCs were washed with 1X FACS buffer. Pellets were mixed with dead cell discrimination dye supplied with the kit and incubated on ice for 15 minutes in dark. The dead cell discrimination dye can only cross the cell membranes of dead cells, where it reacts with cytoplasmic free amines. Finally, cells were washed $2 \mathrm{X}$ with FACS buffer and analyzed within $1 \mathrm{hr}$ by flowcytometry (FACScalibur). The untreated cells, which served as control, are used for defining the basal level of dead cells. The percentage of cells that have been induced to undergo apoptosis is then determined by subtracting the percentage of dead cells in the untreated population from percentage of dead cells in the treated population.

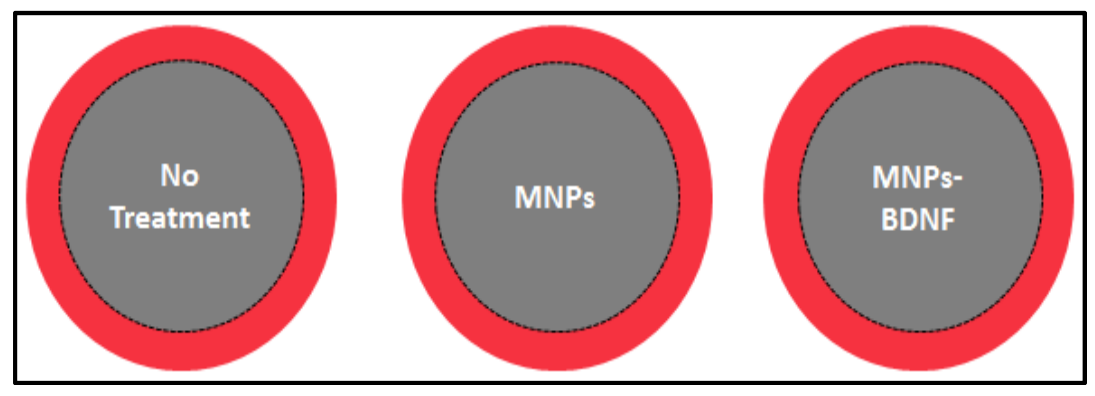

Figure 11: Schematic of the experimental design applied for cell-viability assay with MNPs-BDNF nanoformulations. 


\subsection{Formulation of liposomes-based magnetic nanocarriers:}

\subsubsection{Synthesis of ultrasmall magnetic nanoparticles:}

The ultrasmall $\mathrm{Fe}_{3} \mathrm{O}_{4}$ MNPs were synthesized according to the coprecipitation method proposed by Sun et al (2004), with minor modification. All glassware was cleaned overnight by aqua regia before used for reactions. Briefly, Solution of $3 \mathrm{ml} \mathrm{FeCl} 3(0.487 \mathrm{~g}$ dissolved in $2 \mathrm{~mol} / \mathrm{L} \mathrm{HCl})$ and $10.33 \mathrm{ml}$ $\mathrm{H}_{2} \mathrm{O}$ was stir-mixed with $2 \mathrm{~mL} \mathrm{Na} \mathrm{SO}_{3}(0.126 \mathrm{~g}$ in $2 \mathrm{~mL}$ of water) drop-by-drop within a minute. Upon change of color from yellow to red-light yellow, this solution was mixed into $80 \mathrm{~mL} \mathrm{NH} \mathrm{NH}_{3} \cdot \mathrm{H}_{2} \mathrm{O}(0.85 \mathrm{~mol} / \mathrm{L})$ under vigorous stirring. A black precipitate is quickly formed, which was allowed to crystallize further for approximately 30 minutes under continuous stirring. The suspension was washed and formation of stable MNPs was achieved by adjusting the $\mathrm{pH}$ from 7.5 to 3.0 and temperature from $90^{\circ} \mathrm{C}$ for first five minutes to $100^{\circ} \mathrm{C}$ degree for about one hour. A change in color of dispersion from black to reddish-brown suggests towards compact MNPs which is washed with water for at least three times.

\subsubsection{Formulation of PEGylated magneto-liposome and transferrin conjugation}

Preparation of PEGylated liposomes and encapsulation of MNPs in its core was achieved simultaneously according to the extrusion method (Deng et al., 2012). 7:2:0.5 molar ratios of EPC, Cholesterol, and mPEG2000-DSPE was thoroughly mixed and evaporated under lower pressure. $1 \mathrm{mg}$ ultrasmall MNPs 
was added onto the resultant thin lipid film for rehydration. PEGylated liposomal vesicles containing MNPs is formed which is extruded by handheld extruder (Avanti Polar Lipids, Inc.) containing polycarbonate membrane filter of different pore size $(400 \mathrm{~nm} / 200 \mathrm{~nm} / 100 \mathrm{~nm})$. Prior to extrusions, the extruder was warmed on a hot plate at a temperature above the transition temperature of lipid. The resulting unilamellar magnetized-liposomal nanovehicles of homogenous sizes were separated from unloaded MNPs by centrifugation at 1,000 rpm $\times 2$ minutes. The purified magneto-liposomes were stored at $4^{\circ} \mathrm{C}$ further use. Fluorescent labeling was achieved by adding $0.8 \%$ of CFPE relative to the total lipids into the initial liposome formulation mixture.

Transferrin conjugation was achieved by mixing $0.5 \mathrm{mg}$ fresh transferrin into $200 \mu \mathrm{L}$ of prepared magnetic-liposome at $37^{\circ} \mathrm{C}$ for $\sim 30 \mathrm{~min}$ [36].

\subsubsection{Validation of Transferrin conjugation:}

Conjugation of transferrin in the periphery of magneto-liposomes was determined spectrophotometrically with UV-visible spectrophotometer (Varian, USA). Presence of transferrin-specific absorbance was measured by scanning wavelength range from $250-400 \mathrm{~nm}$.

\subsubsection{Determination of encapsulation efficiency (EE)}

The encapsulation efficiency value designates the percentage of MNPs loaded in liposomes and is given by the ratio between the iron concentration in magneto-liposomes and that feeded into the encapsulation-mixture in the beginning of formulation process. It is calculated as: 
$\mathrm{EE}(\%)=($ Iron concentration in magneto-liposome $) /($ Iron concentration feeded in beginning of encapsulation process) $\times 100$

\subsubsection{Quantitation of encapsulated MNPs in liposome}

Ammonium thiocyanate-based photometric assay was used to determine the amount of encapsulated $\mathrm{Fe}_{3} \mathrm{O}_{4}$. Magneto-liposomal formulation was mixed with Triton X-100 $(1 \%, v / v)$ in a ratio of $1: 1.5$ causing de-encapsulation of MNPs. One volume concentrated $\mathrm{HCl}(37 \%)$ was added to this mixture and incubated at $60^{\circ} \mathrm{C}$ for $4 \mathrm{hrs}$. The suspension was centrifuged at $12,000 \mathrm{~g}$ for 10 minutes and supernatant was collected for quantification of iron oxide. Equal ratio of supernatant and $1 \%$ ammonium persulfate solution was mixed with one volume $0.1 \mathrm{M}$ Ammonium thiocyanate and incubated at RT for 5 minutes. The absorption of resulting red iron-thiocyanate was measured at $490 \mathrm{~nm}$ by a microplate reader (Synergy HT, Multi-mode microplate reader, BioTek Instrument, Inc., Winooski, Vermont, USA). A standard curve was prepared with known concentrations of $\mathrm{Fe}_{3} \mathrm{O}_{4}$ in $\mathrm{H}_{2} \mathrm{O}$.

\subsubsection{Determination of colloidal- and fluorescent-integrity of magneto-} liposomes:

The hydrodynamic size of freshly prepared magneto-liposomes was determined by DLS (90 Plus Particles Size Analyzer, Brookhaven Instrument Corp., USA). The nanocarriers were stored at $4^{\circ} \mathrm{C}$ and durability of their structural-integrity was evaluated by measuring the size by DLS for up to 28 days. Further, their probable stability in peripheral-circulation was assessed by 
measuring the size at body temperature and in an in vitro closed circulatory system. Magneto-liposomes were dispersed in the mixture of ECM and AM (1:1), incubated at $37^{\circ} \mathrm{C}$ for up to $28 \mathrm{hrs}$, and used for DLS measurement. To analyze the sustainability of magneto-liposomal integrity in blood circulation, an in vitro closed circulation system was set up using a bidirectional, self-priming peristaltic pump (Mini Pump Peristaltic Pump Variable Flow C-2 Lab Pump, Fisher Scientific). The nanoformulation was allowed to circulate in a $0.8 \mathrm{~mm}$ capillary for 120 times at a flow rate of $1 \mathrm{ml} / \mathrm{min}$. The circulating medium was prepared with 5\% dextran-500 (Cat \#: 50-247-495, Thermo Fisher Scientific, Boston, US) in PBS which makes a viscosity equivalent to that of blood (4.5 centipoise). Samples were collected after $10,30,60$, and 120 circulations and subjected to the size analysis by DLS.

The fluorescent-integrity of magneto-liposome was determined by measuring the fluorescent intensity of associated CFPE at wavelength 485/20nm-528/20 nm (Ex/Em) by microplate reader (Synergy HT, Multi-mode microplate reader, BioTek Instrument, Inc., Winooski, Vermont, USA).

\subsection{In vitro blood-brain barrier (BBB) and nanocarrier transmigration:}

\subsubsection{Preparation of in vitro BBB model:}

The primary human brain microvascular endothelial cells (HBMEC) and human astrocyte (HA) cells were cultivated as per provider's recommendations The BBB model was established as described earlier by Gandhi et al. (2010). The in vitro BBB model is developed in a bi-compartmental transwell culture plate 
(Product \# 3415, corning life sciences, Mexico). The upper chamber of this plate is separated from the lower one by a $10 \mu \mathrm{m}$ thick polycarbonate membrane possessing $3.0 \mu \mathrm{m}$ pores. In a sterile 24 -well cell culture plate with pore density of $2 \times 10^{6}$ pores $/ \mathrm{cm}^{2}$ and cell growth area of $0.33 \mathrm{~cm}^{2}, 2 \times 10^{5} \mathrm{HBMEC}$ and HA were grown to confluency on the upper chamber and underside of lower chamber respectively. Intactness of in BBB was determined by measuring the transendothelial electrical resistance (TEER) using Millicell ERS microelectrodes (Millipore). A mean TEER value of $\sim 200 \mathrm{ohms} / \mathrm{cm}^{2}$ cell culture insert is considered consistent with the formation of the BBB.

\subsubsection{Transmigration of fluorescent magneto-liposomes across in vitro BBB model:}

Transmigration study of magneto-liposomal nanoformulations was conducted on the $5^{\text {th }}-6^{\text {th }}$ day of the BBB culture when ideal integrity of this membrane was achieved as established by TEER measurement. Equal quantity of transferrin conjugated and/or unconjugated fluorescent magnetic-liposomes were added to the apical chamber and incubated at $37^{\circ} \mathrm{C}$ in the presence or absence of a magnetic force of $0.03-0.08$ Tesla placed externally below the trans-well's basolateral chamber. Samples were collected from both the chambers at different time points and Fluorescent intensity was measured at wavelength $485 / 20 \mathrm{~nm}-528 / 20 \mathrm{~nm}(\mathrm{Ex} / \mathrm{Em}$ ) by microplate reader (Synergy HT, Multi-mode microplate reader, BioTek Instrument, Inc., Winooski, Vermont, USA). 
The relative apparent permeability coefficient was calculated from the equation as:

Relative $\mathrm{Papp}=($ Fluorescent intensity in basal chamber $) /($ Fluorescent intensity in apical chamber) $\times 100$

Where Papp: apparent permeability

\subsubsection{Transmigration and efficiency of BDNF:}

The in vitro $B B B$ were established in bi-compartmental transwell culture plate. Free and MNPs bound BDNF were added in upper well and a week magnet was placed on the basal side of the plate. After $48 \mathrm{hr}$ exposure of magnetic force, samples were collected from the basal chamber and BDNF transmigration ability was analyzed by BDNF ELISA as explained above.

The functional efficiency of transmigrated BDNF was determined by its ability to induce CREB expression. Astrocytes were collected from the basal side of in vitro BBB and used for total RNA extraction, cDNA synthesis and qPCR as described above.

\subsubsection{Cytotoxicity assay:}

Cytotoxicity was assessed by MTS cell viability assay using CellTiter $96 \circledast$ Aqueous one solution cell proliferation assay kit (Catalog \# G 3580, Promega, USA). HBMEC and HA cells were seeded in 96-well tissue plates at a density of $5 \times 10^{3}$ cells/well. After $24 \mathrm{hr}$, culture medium was replaced with $100 \mu \mathrm{l}$ fresh media containing different concentrations of $\mathrm{Fe}_{3} \mathrm{O}_{4}$ (from0.02-0.5 $\mathrm{mgmL}^{-1}$ ). 
$20 \mu \mathrm{L}$ of MTS solution was added into each well 24 and $48 \mathrm{hr}$ post-treatment and incubated at $37^{\circ} \mathrm{C}$ for $2 \mathrm{hr}$. Absorbance was recorded at $490 \mathrm{~nm}$ by microplate reader (Synergy HT, Multi-mode microplate reader, BioTek Instrument, Inc., Winooski, Vermont, USA) 


\section{Results and discussion:}

The brain is one of the common target organs for HIV and recreational drugs. Abusive drug is a risk factor for HIV-1 infection and AIDS progression (Nath et al., 2002; Burdo et al., 2006; Hauser et al., 2007; Ferris et al., 2008; Rogers, 2011). Approximately 230 million are illicit drug users and it will increase by $25 \%$ in next 3-4 decades $\left(\mathrm{UNODC}_{\mathrm{b}}, 2012\right)$. Opiates are among the most abused drugs. About 26-36 million aged 15-64 have been estimated to use opioids in 2010 $\left(\mathrm{UNODC}_{\mathrm{b}}, 2012\right)$. It stimulates the $\mu$ opioid receptors that promote growth of HIV and potentiate the HIV-related neurotoxicity (Nath et al., 2000, 2002; Hauser et al., 2005). Despite significant advances in antiretroviral therapy, the worldwide HIV morbidities, mortalities and associated NeuroAIDS continue. This is attributed to the inability of the ARV drugs to completely eliminate HIV reservoirs especially in the brain, which is mainly as a result of the impenetrability of most of the ARV drugs across the BBB. Many of the new chemical entities, that do not cross BBB under normal condition, aimed at treating brain disorders have failed clinically because of their impenetrability across BBB. In recent years, the use of magnetic nanoparticles has created significant interest in biomedicine and biomedical engineering for applications including magnetic nanocarriers for drug delivery systems and contrast enhancement agents in MRI for diagnostics. As such, we investigated a novel magnetic nanoparticle based drug delivery system for delivery of $\mu$ opioid receptor antagonist (CTOP, a $\mu$ opioid receptor blocker), neuroprotective agent (BDNF), and ARV drugs across the BBB. 


\subsection{Characterization of magnetic nanoparticles:}

Size and morphology of MNPs were characterized by TEM. A typical TEM micrograph of magnetic particles is shown in Figure 12. It shows that the average size of magnetic particles is about $25-40 \mathrm{~nm}$. It should be noted that the higher surface to volume ratio enhances target-affinity of MNPs in comparison to the micro-sized magnetic particles and can even manipulate and target at the subcellular organelles levels.
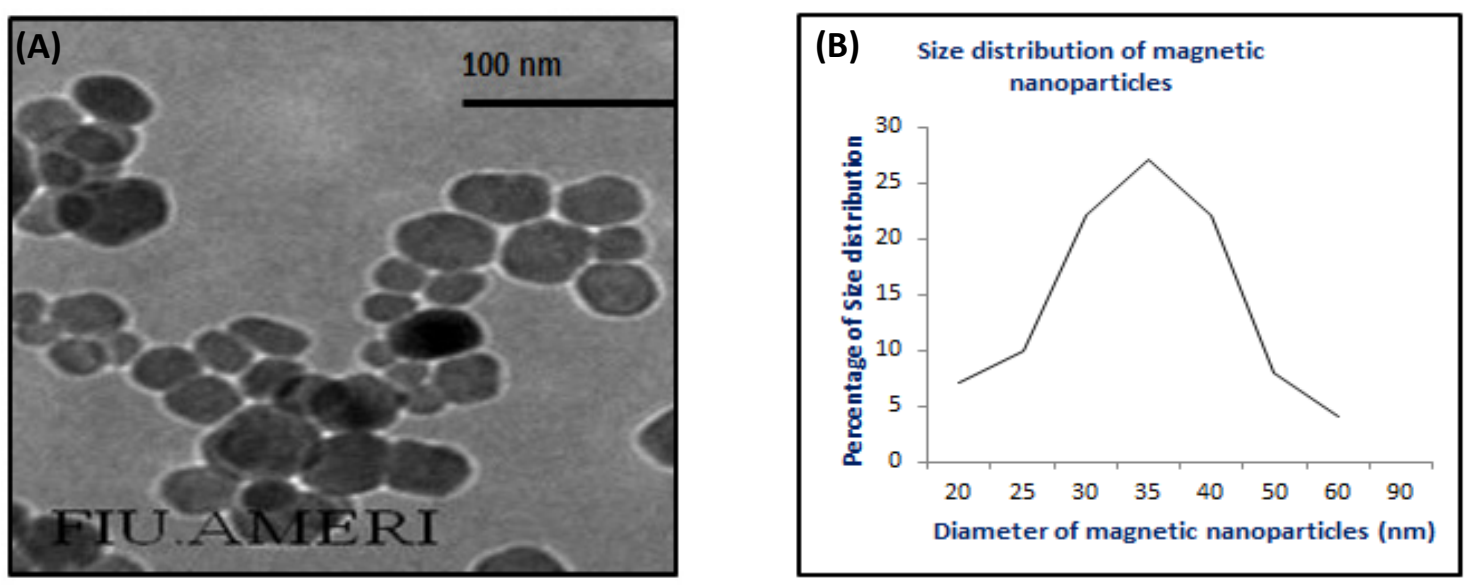

Figure 12: (A) Transmission electron micrograph of $\mathrm{Fe}_{3} \mathrm{O}_{4}$ magnetic particles. (B) Size distribution of magnetic nanoparticles: Average size of particles is $25-40 \mathrm{~nm}$.

It has been established that smaller particles $(<10 \mathrm{~nm})$ are lost to extravasation and larger particles (>200 nm) are quickly captured and excreted. Particles between $10-70 \mathrm{~nm}$ have been shown to penetrate capillary vessels. Thus, characterized MNPs can be compatible for enhanced drug delivery across the tightly junctioned BMECs along the capillaries lining throughout the cerebral microvasculature. Furthermore, In view of the fact that particles between $70-200$ $\mathrm{nm}$ possess longer blood circulation time, MNPs of $25-40 \mathrm{~nm}$ size could be 
successfully hybridized with liposomes for synthesis of magnetoliposomes of about $100 \mathrm{~nm}$ which will enhance the bioavailability of associated drugs. Importantly, MNPs of about $30 \mathrm{~nm}$ size exhibit superparamagnetism and can respond to an external magnetic field. Thus, it is possible to "remote control" the movement of drug loaded nanoparticles for target-specific delivery by applying the magnetic force at the exterior of desired site.

\subsection{Effect of $\mathrm{pH}$ on surface charge distribution of MNPs:}

Aqueous solutions of MNPs such as $\mathrm{Fe}_{3} \mathrm{O}_{4}$ perform amphoterism because of adsorption of amphoteric hydroxyl $(-\mathrm{OH})$ group and develop positive or negative charges at the magnetite-water interface in $\mathrm{pH}$-dependent manner (Tombacz et al., 2006). The flexibility in the surface charge allow binding of wide range of molecules either via direct, but week, ionic interactions to the MNPs (Saiyed et al., 2009) or via surface coating or tethering agents (Yoo et al., 2012; Wiogo et al., 2012). Thus, adsorption efficiency of a molecule on surface of MNPs may be influenced by $\mathrm{pH}$ of reaction mixture. The magnitude of charge at the surface of colloidal system is quantified by zeta potential using the zeta analyzer. The measure zeta potentials at the surface of MNPs in different $\mathrm{pH}$ range of Tris-EDTA buffer are shown in figure 13A. Similar to previous reports, our results show that the isoelectric point $(\mathrm{p} /)$ of MNP is about 7.0 and have positive and negative charge below and above pl. We get a significant negative zeta potential value $(-20.93)$ at $\mathrm{pH} 7.4$, which is also the physiological $\mathrm{pH}$ range. Thus, it is possible that at $\mathrm{pH}$ 7.4 MNPs may have sufficient charge for the 
adsorption of drug molecules. A model for electrostatic interaction between MNPs and different drugs has been illustrated in figure 13B. Different drugs possess reactive free functional groups such as $\mathrm{OH}, \mathrm{NH}_{2}$, etc. These functional groups may gain charges such as $\mathrm{OH}_{2}{ }^{+}$and $\mathrm{NH}_{3}{ }^{+}$due to change in $\mathrm{pH}$ of aqueous media and may influence peptide amphiphilic properties. Therefore, negative charge on the surface of MNPs at $\mathrm{pH} 7.4$ and possible positive charges moieties in the drugs may allow direct binding via ionic interaction. The binding of molecules to MNPs can be reversible due to $\mathrm{pH}$ variation, which may allow the bound drugs to be released at the target site.
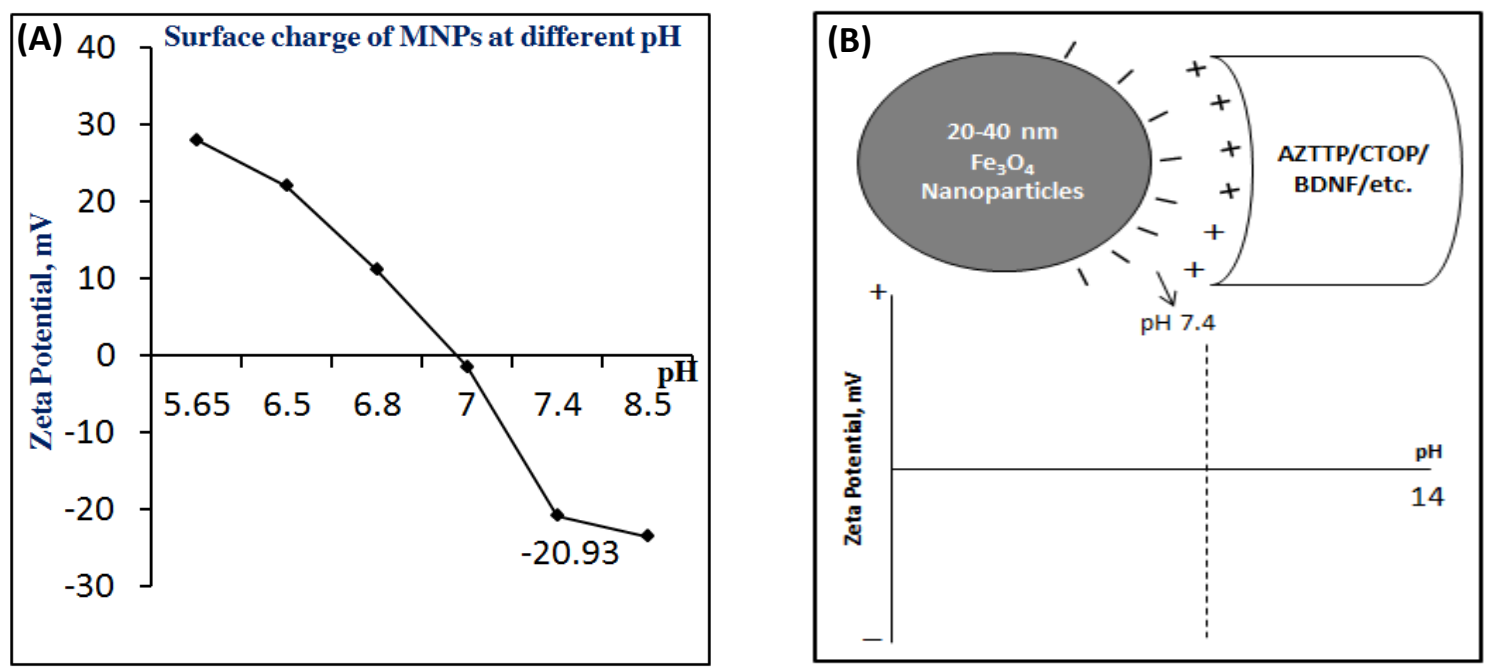

Figure 13: (A). Zeta potential (surface charge) of MNPs at different pH. (B). Schematic illustration of proposed electrostatic interaction between MNPs and different drugs at $\mathrm{pH} 7.4$.

\subsection{CTOP adsorption on MNPs surface:}

Infrared spectra obtained via Fourier transform infrared spectroscopy (FTIR) can help in identifying the bonding present in an unknown molecule via measuring the bending vibration of certain functional groups that are present in 
the sample (Balaji et la., 2009). In infrared spectroscopy, IR radiation is passed through a sample. Some of the infrared radiation is absorbed by the sample and some of it is passed through (transmitted). The resulting spectrum represents the molecular absorption and transmission, creating a molecular fingerprint of the sample. Like a fingerprint no two unique molecular structures produce the same infrared spectrum.
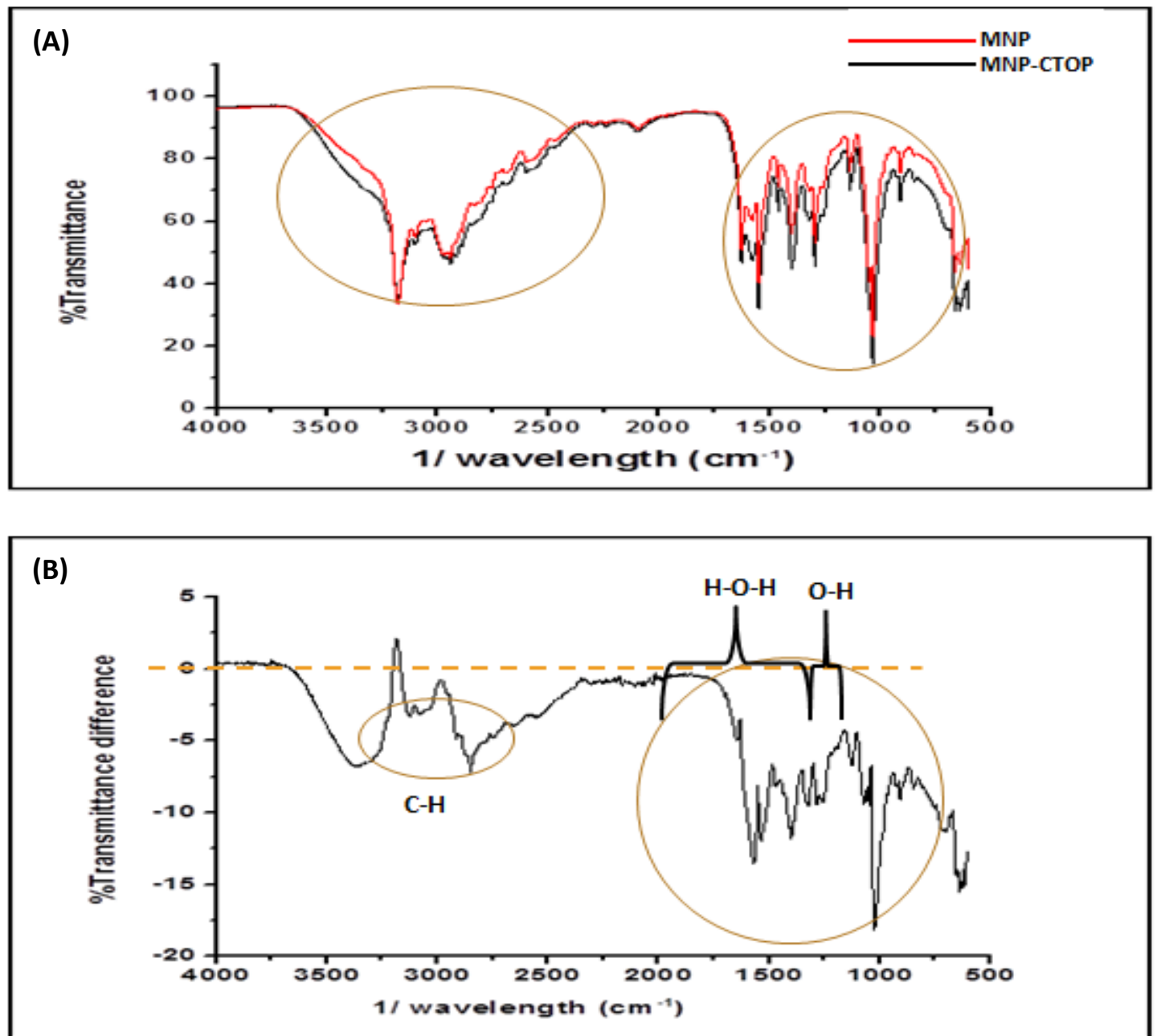

Figure 14: FTIR spectra of transmittance: (A) \% transmittance of MNP and "MNP+CTOP". (B). Difference between \% transmittance of MNP and "MNP+CTOP": transmittance of "MNP+CTOP" obtained from FTIR were subtracted from transmittance of MNP only and difference in transmittance at specific band ranges were co-related with presence or absence of functional group associated with CTOP and aqueous medium. 
Fourier Transform Infrared Spectroscopy (FTIR) spectra were performed to the lyophilized MNP bound with or without CTOP using a FTIR spectrophotometer in wave range of $4000-400 \mathrm{~cm}^{-1}$ (Figure 14A). Typically, bending vibration at about $900-1000 \mathrm{~cm}^{-1}$ corresponds to the $\mathrm{O}-\mathrm{H}$ bond, particularly for strong hydrogen bridges. Similarly, about $1000-1600 \mathrm{~cm}^{-1}$ is typical of the H-O-H molecule (Lopez et al., 2010). In order to define the presence of CTOP on MNPs, transmittance spectra of "MNPs-CTOP" were subtracted from that of MNPs only and percent transmittance difference were plotted (Figure 14B). Maximum variation in transmittance was detected at about $600-1600 \mathrm{~cm}^{-1}$. We can see a reduction in transmittance up to $18 \%$ at $1025 \mathrm{~cm}^{-1}$ for CTOP bound MNPs. Thus, change transmittance for "MNP+CTOP" in compare to MNPs at these bands may correspond to two interrelated phenomenon. First, hydroxyl groups from water molecule may have attached by the hydrogen bonds in the iron oxide surface influencing the negative charge distribution on surface which is reflected as higher negative zeta potential. Similarly, water molecules $(\mathrm{H}-\mathrm{O}-\mathrm{H})$ may have chemically adsorbed to the magnetic particle surfaces, again influencing the surface charge. Second and more importantly, presence of charge due to $\mathrm{O}-\mathrm{H}$ or $\mathrm{H}-\mathrm{O}-\mathrm{H}$ on the surface of MNPs might have allowed binding of CTOP resulting in change of bending vibration of bonds which may be reason of reduced transmittance. Additionally, we noticed that transmittance of "MNP+CTOP" is also reduced up to $7 \%$ at about $2870 \mathrm{~cm}^{-1}$ and $3370 \mathrm{~cm}^{-1}$ which may reflect increased absorption at these bands due to presence of additional $\mathrm{CH}$ and $\mathrm{NH}_{2}$ group of CTOP. Typically, frequency 
range for $\mathrm{N}-\mathrm{H}$ group is $3300-3500 \mathrm{~cm}^{-1}$ and that of $\mathrm{C}-\mathrm{H}$ is $2700-3300 \mathrm{~cm}^{-1}$. CTOP absorption on the surface of MNPs was further verified using fluorescent-based detection method. The Alexa flour 610 succinimidyl (NHS) esters were used for tagging of this peptide. The NHS ester mediated bonding is most efficient and convenient way to attach fluorophores to amine-containing ( $\mathrm{R}-\mathrm{NH} 2)$ molecules such as peptides, proteins, or amine-modified nucleic acids. The stability of amide bonds formed in the reaction is as good as that of peptide bonds. Thus, selective linking of fluorophores to peptides opens window for many purposes such as quantification, imaging, etc. As shown in figure 15, significant fluorescent intensity were detected on the MNPs which were immobilized with dye tagged peptide. Simultaneously, dye-exposed or non-exposed MNPs showed no trace of fluorescent activity. This suggests that CTOP could successfully be immobilized on the surface of MNPs.

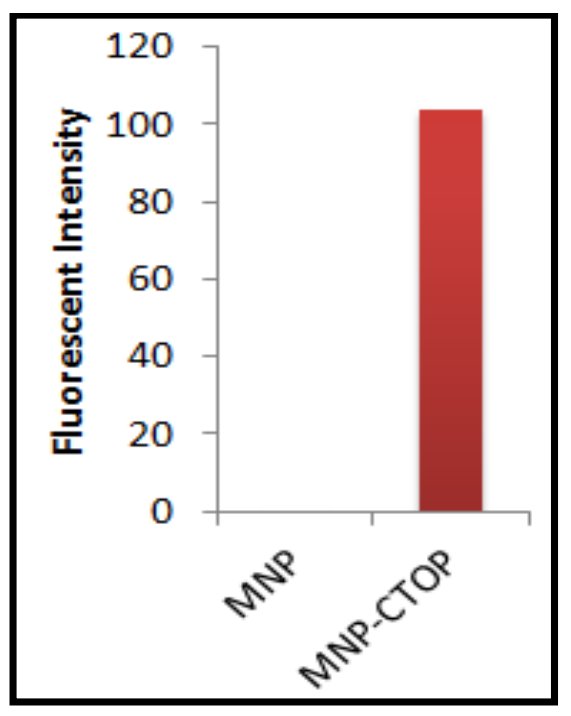

Figure 15: Fluorescent-based CTOP binding verification: Immobilization of red fluorescent dye tagged CTOP on MNPs emits fluorescence while MNPs without CTOP shows no fluorescence. 


\subsection{Time kinetics and binding isotherm of CTOP to MNPs:}

Data presented in the figure $16 \mathrm{~A}$ and $16 \mathrm{~B}$, shows the time kinetics and percent direct binding of CTOP to MNPs. We found a significant binding of CTOP to magnetic nanoparticles. The MNPs were dispersed in Tris-EDTA buffer ( $\mathrm{pH}$ 7.4) and mixed with CTOP. The mixture was incubated in tube rotator at room temperature and supernatant were collected at different time points from 5-240 minutes (Figure 16A). The unbound fraction of CTOP present in supernatant was quantified by HPLC. The difference between the total CTOP added and unbound CTOP was used to calculate the amount of CTOP bound to the MNPs. Maximum binding was achieved at about 5 minutes of incubation which remained unaffected till 4 hour of experimental duration. After the time kinetics of binding was optimized, the CTOP binding efficiency was estimated by using different ratios (Weight/Concentration),1:0.16, 1:0.33, 1:0.66, 1:1, and 1:1.33 of MNPs and CTOP, respectively. Data presented in Figure 16B show the adsorption isotherm of CTOP on MNPs. The result obtained from three independent experiments indicates a maximum binding efficiency of about $140 \mu \mathrm{g}$ CTOP per mg of MNPs. It should be noted that time kinetics and loading efficiency significantly affect the drugs sustainability and safe dosing. This eventually influences drugs bioavailability. The sustainable binding of CTOP on MNPs for over four hours is in accordance to our hypothesis where, under the non-invasive magnetic influence, drug delivery to the target could be maximized before it leaches out of the nanocarrier. 

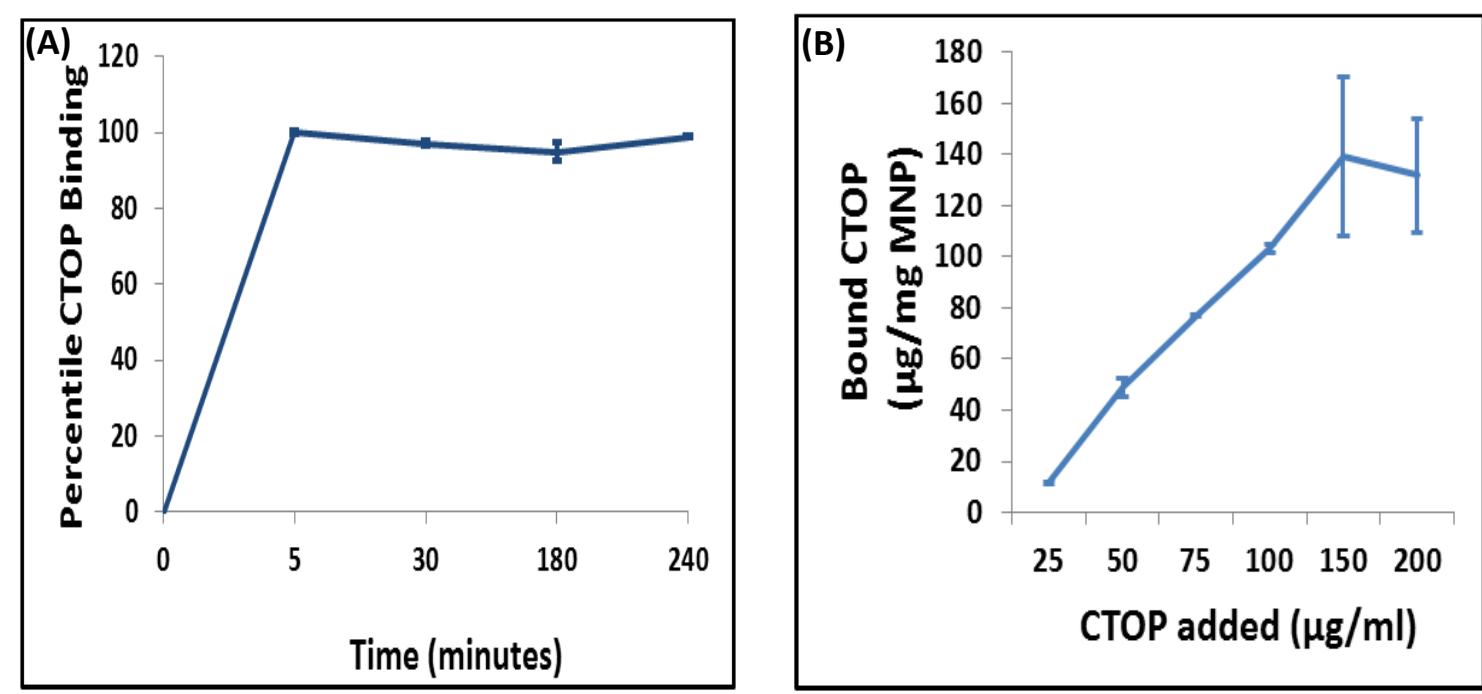

Figure 16: (A). Time kinetics of CTOP binding onto MNPs. (B). Binding isotherm for CTOP on MNPs.

\subsection{Functional efficiency of MNPs bound CTOP:}

\subsubsection{Inhibition of morphine-induced peripheral pathogenesis:}

Exposure of morphine has been shown to modulate functions of various immune cells such as phagocytes, T cells, dendritic cells, etc. (Eisenstein and Hilburger, 1998; Messmer et al., 2006; Rivera-Amill et al., 2010; Saurer et al., 2006; Wang et al., 2008). It significantly alters the expression of cytokines, chemokines, etc. and induces apoptosis in both peripheral and neuronal cells. Studies from our lab (Nair et al., 1988) have shown that morphine exposure causes significant induction of apoptosis in PBMCs. Though different kinds of opioid receptors exist, morphine exerts its effect through the $\mu$ opioid receptor. Therefore, use of a $\mu$ opioid receptor antagonist could prevent the morphineinduced effect and may provide significant therapeutic benefits. We used D-PenCys-Tyr-DTrp-Orn-Thr-Pen-Thr-NH2 (CTOP), which is a highly selective and 
potent $\mu$ receptor antagonist and remains impenetrable through the Blood Brain Barrier (BBB). To compare the efficiency of MNPs bound CTOP with that of free CTOP, PBMCs were treated with morphine and its effect on apoptosis induction was analyzed using flow-cytometry (Figure 17). Annexin-V is the indicator protein of the earliest events in apoptosis. As expected, more than $80 \%$ of PBMCs were found to be Annexin-V positive when they were treated with morphine (figure $17 \mathrm{C}$ and 17F). This effect was significantly reversed when cells were treated with free or MNPs bound CTOP.

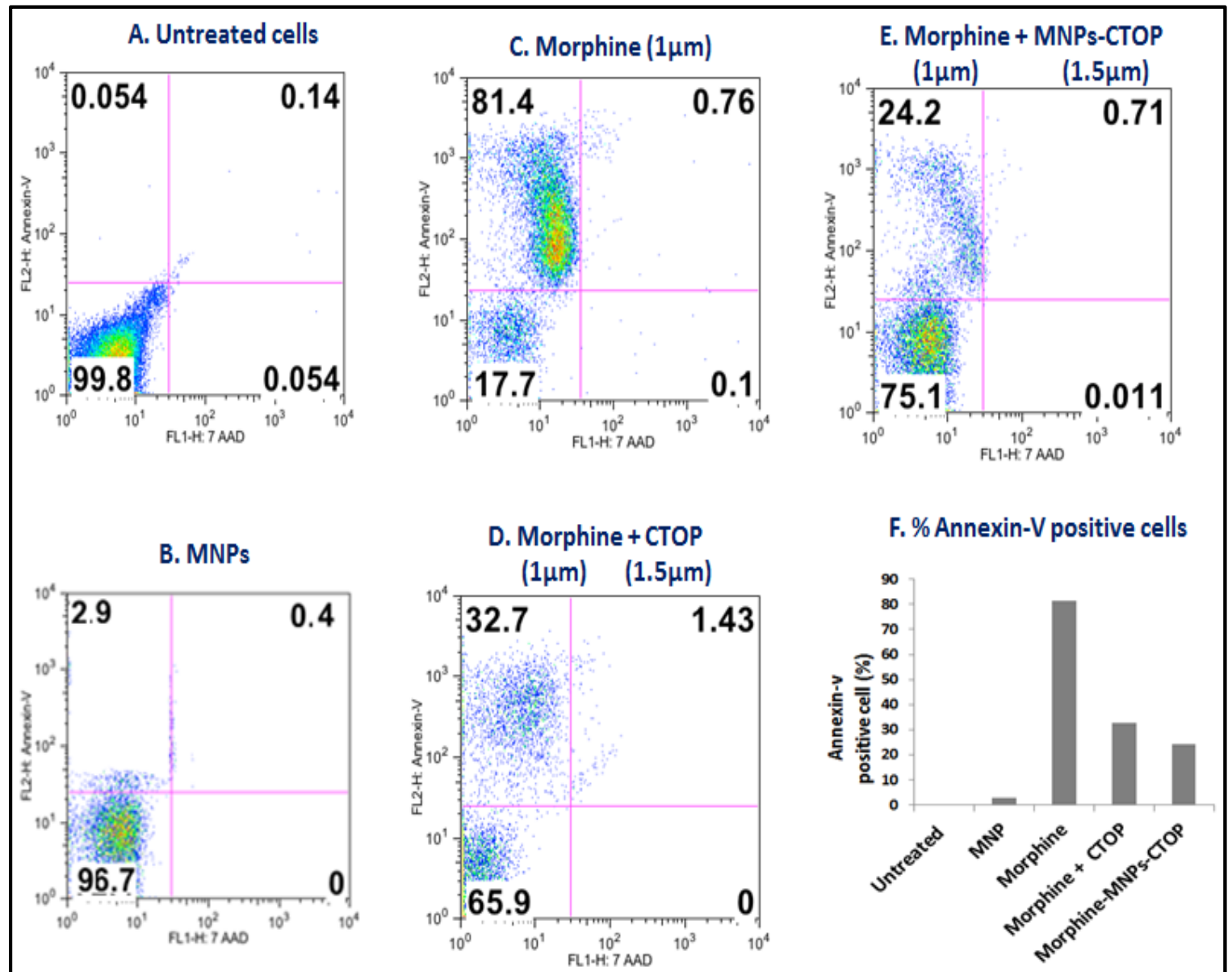

Figure 17: Flow-cytometry to evaluate the efficacy of MNPs bound CTOP on morphine induced apoptosis in PBMCs: MNP bound CTOP (E) possess parallel efficacy to that of free CTOP (D) in suppressing the apoptosis induced by morphine (C). 
Number of Annexin-V positive cells was reduced approximately by $50 \%$ upon treatment with free CTOP and only $32 \%$ cells were found Annexin-V positive. Similarly, MNPs-bound CTOP exerted equivalent apoptosis inhibition efficiency and nearly $24 \%$ cells were found Annexin- $V$ positive in this case. Untreated or only MNPs treated cells showed near zero or insignificant induction of apoptosis. Thus, our result suggests that efficacy of CTOP upon its binding to MNPs is preserved.

\subsubsection{Inhibition of morphine-induced neuronal pathogenesis:}

Morphine crosses the BBB and believed to suppress CNS immune responses by various mechanisms. It inhibits or downregulates various inflammation-suppressing chemokines and cytokines such as macrophage inflammatory protein, interleukin-8 etc (Mahajan et al., 2002). Also, morphine have been shown to induce apoptosis in various CNS cells such as microglia, astrocytes, neurons, etc. (Goswami et al., 1998; Yin et al., 1999; Hu et al., 2005). All these immune-inhibitory effect of morphine could influence neuroinflammation leading to neuropathogenesis. In fact, deregulation of chemokine or cytokine expression in CNS cells is a hallmark phenomenon associated with neuronal degeneration. As such, morphine is putatively believed to acts synergistically as a co-factor in neuropathogenesis. In particular, morphine has been shown to synergize the HIV infection associated neurocognitive disorders where spinal architecture of neuronal cells is significantly altered (Atluri et al., 2013). Figure 18 shows altered spinal morphology of neuroblastoma cells upon morphine treatment. This alteration of spinal architecture may negatively affect 
the synaptic plasticity during morphine exposure. Spine morphology play important role in maximizing the effectiveness of the synaptic transmission leading to cognitive modulation. Neuronal adaptation pattern is differentially regulated during opioid addiction (Simonato, 1996) and cause rapid development of tolerance, physical and psychological dependence. These opioid-dependence associated disorders could significantly be diminished by supplementation of anti-opioid agents which may prevent opioid-induced pathogenesis. However, current treatments to alleviate the action of opioids are less effective at CNS level, basically due to impermeability of therapeutic molecules across blood-brain barrier. As a first step towards our hypothesis in developing MNPs-based nanoformulations of anti-opioid agents, we analyzed the efficiency of MNPs bound CTOP in preventing the morphine induced inhibition of spinal density as a sign of neuronal degeneration (Figure 19).

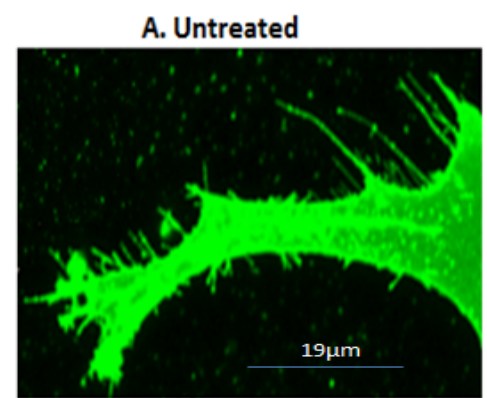

D. MNPs

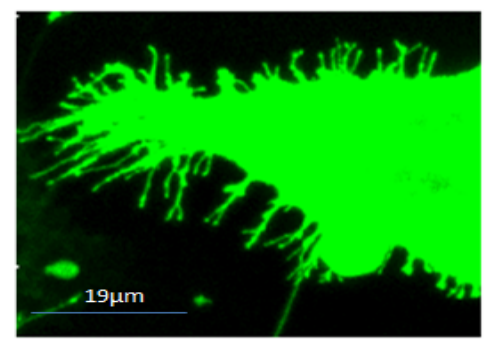

B. Morphine

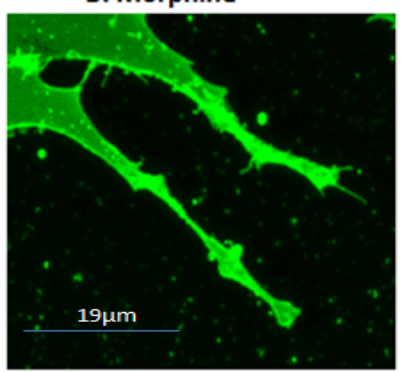

E. Morphine + CTOP

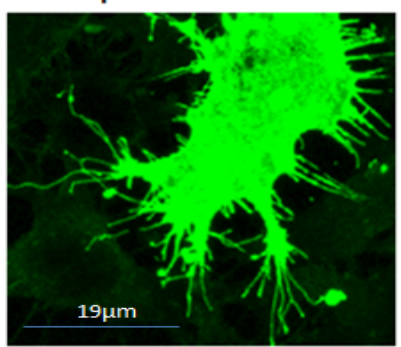

C. Morphine + MNPs

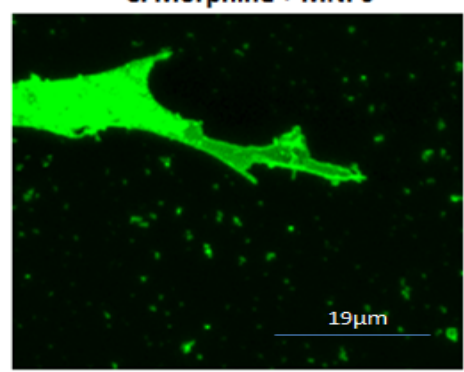

F. Morphine + MNPs-CTOP

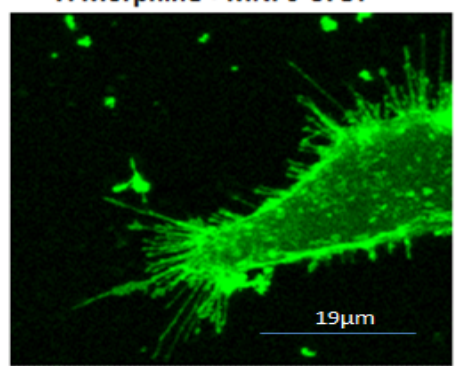

Figure 18: Confocal microscopy to evaluate the efficacy of MNPs bound CTOP on morphine induced neuropathogenesis: Free $(E)$ and MNP bound CTOP $(F)$ prevents the morphine induced spinal degradation in neuroblastoma cells, SK-N-MC (B). 


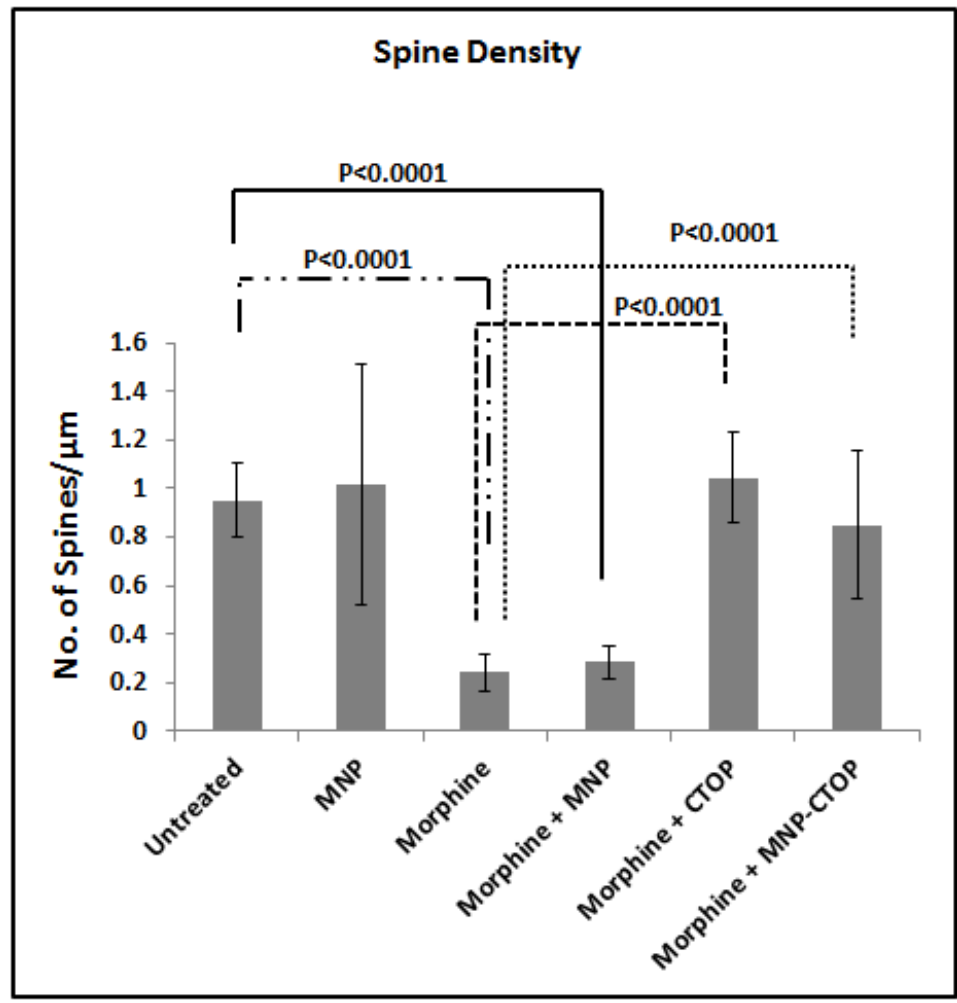

Figure 19: Spinal density (No. of spines/ $\mu \mathrm{m}$ dendritic length) of SK-N-MC showing morphine induced spinal degeneration and effect of Free and MNP bound CTOP on prevention of this degradation.

As shown in figure19B, morphine treated cells showed a spinal density of $0.24 \pm 0.07$ per $\mu \mathrm{m}^{2}$, whereas the same in untreated cells were approximately 1 per $\mu \mathrm{m}^{2}(\mathrm{P}<0.0001)$. This significant decrease in morphine-induced spinal density is prevented upon CTOP treatment. Both free and MNPs-bound CTOP showed equivalent efficiency in checking the spinal degeneration (Figure 19; 18E and 18F). Cells with free CTOP and MNPs bound CTOP showed an average spine density of $1.04 \pm 0.18$ and $0.84 \pm 0.30$ per $\mu \mathrm{m}^{2}$ respectively. These values were comparable to that of untreated cells; however significantly higher than that of morphine treated cells only $(P<0.0001)$. Treatment of MNPs alone in cells exposed or non-exposed to morphine (figure19; $18 \mathrm{C}$ and 18B.) did not alter the spinal density. Thus, our result suggests that, similar to their efficacy in 
suppressing apoptosis induction in PBMCs, MNPs bound CTOP possesses parallel effect to that of free CTOP in preserving the neuropathogenesis.

\subsubsection{Inhibition of morphine-induced neuronal pathogenesis during HIV infection:}

As discussed previously, opioids act in synergy with HIV viral proteins and cause greater immunosuppression. Regions of brain with higher opioid receptors such as striatum and hippocampus have been shown to possess increased viral titers (Nath et al., 2000; 2002; Hauser et al., 2005) which lead to faster neuropathogenesis. As such, we analyzed the efficiency of MNPs-bound CTOP in preventing the additive neuro-degeneration of morphine during HIV infection. As reported previously from our laboratory (Atluri et al., 2013), HIV infections lead to severe loss of spinal architecture in neuroblastoma cells (Figure 20B). In compare with uninfected cells where spinal density was approximately 1 per $\mu \mathrm{m}^{2}$, HIV infections reduce the spinal density to $0.35 \pm 0.17$ per $\mu m^{2}(P<0.0001)$ and, though not significant, it goes further down to $0.30 \pm 072$ per $\mu m^{2}$ when exposed to morphine during infection (figure20B, 20C; 21). To analyze the efficacy of our MNP-CTOP nanoformulation in prevention of morphine-induced additive neuropathogenesis, morphine exposed, HIV infected cells were treated with MNPs bound CTOP. As shown in figure 20D and 21, this significantly prevented the spinal degeneration. The spinal density significantly went up to $0.74 \pm 078$ per $\mu \mathrm{m}^{2}$ in compare to HIV infection $(\mathrm{P}<0.0002)$ or co-treatment of morphine with infection $(P<0.0001)$ (Figure 21). 


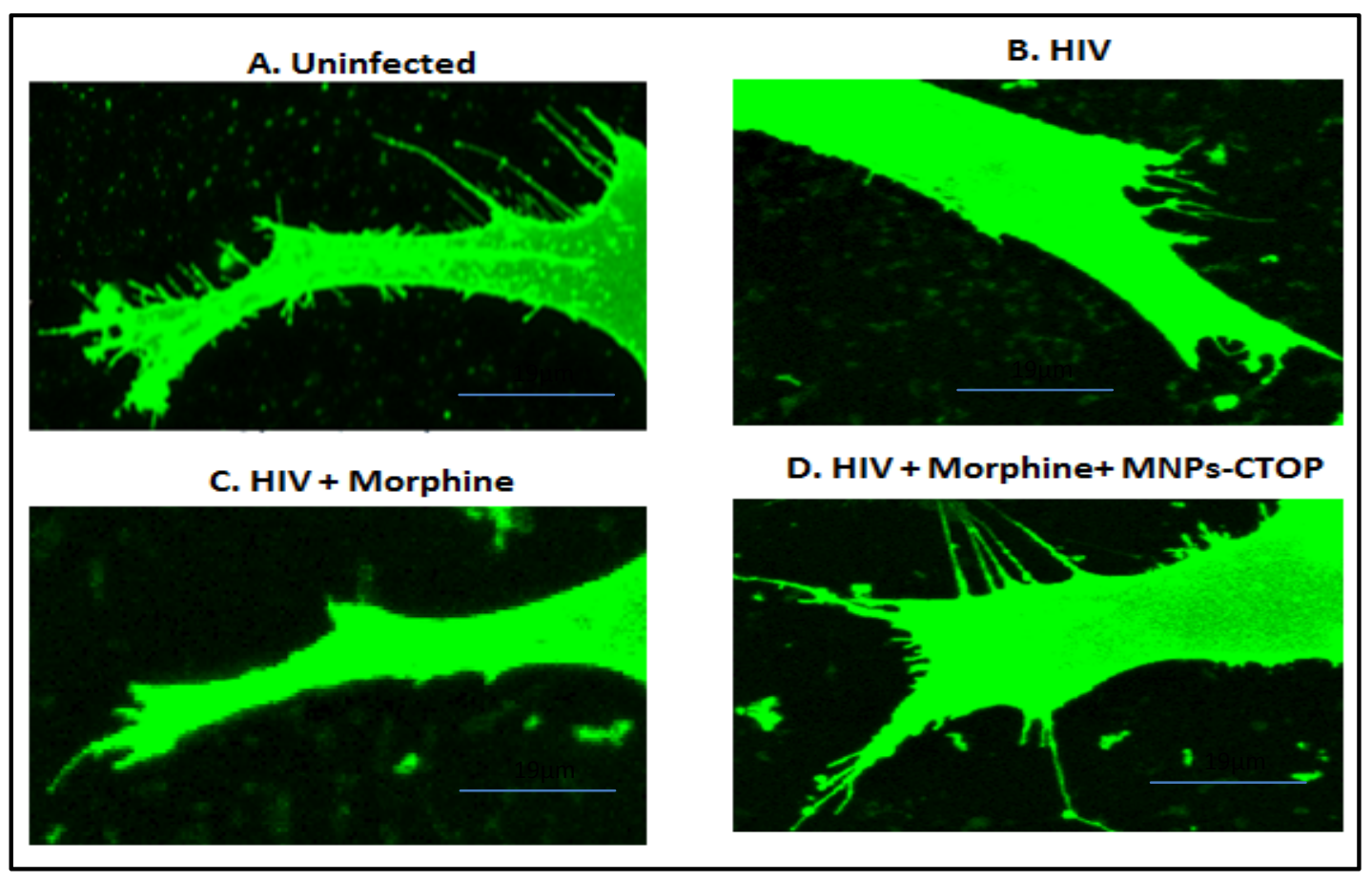

Figure 20: Confocal microscopy to evaluate the efficacy of MNPs bound CTOP on morphine and HIV co-infection induced neuropathogenesis: MNP bound CTOP (D) prevents the spinal degradation in HIV-infected, morphine co-treated neuroblastoma cells, SK-N-MC.

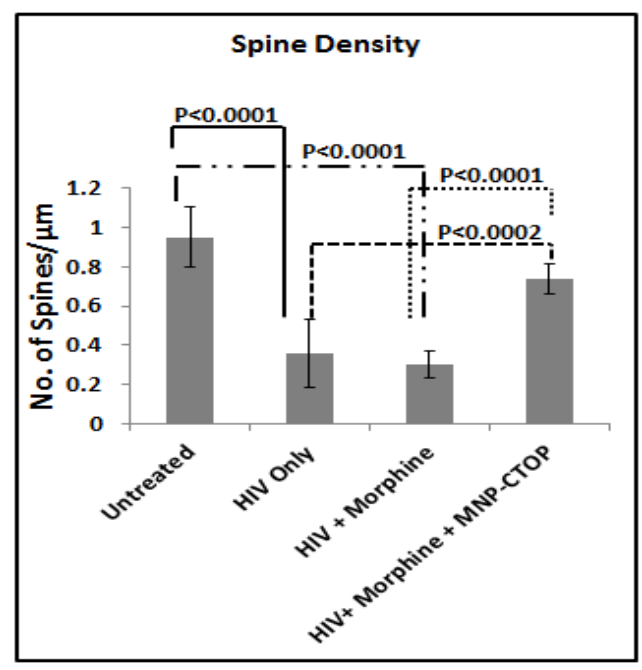

Figure 21: Spinal density (No. of spines/ $\mu \mathrm{m}$ dendritic length) of SK-N-MC showing morphine and HIV coinfection induced spinal degeneration and effect of MNP bound CTOP on prevention of this degradation.

Upregulation of $\mu$ opioid receptor and associated alteration in the expression of pro- and antiapoptotic molecules, cytokines, and chemokines is a common phenomenon during the HIV infection and opioid exposure (Peterson et 
al., 1994; Rojavin et al., 1993; Zhang et al., 2005; Beltran et al., 2006; El-Hage et al., 2005). Apoptosis due to treatment with HIV-1 pathogenic protein gp120 and morphine in $\mu$-opioid receptors deficient mice gets lowered significantly in compare with the wild types (Moorman et al., 2009). The magnetic nanoformulations carrying $\mu$-opioid receptor antagonist, CTOP, could possibly block this receptor and, thus, in turn, may minimize the neuro-pathogenesis exacerbated due morphine co-treatment and/or HIV infection. Therefore, as our result suggests (Figure19-21), higher spinal density in HIV infected and morphine co-treated neuronal cells, upon exposure of MNP bound CTOP, should be a natural outcome than cells where no CTOP was supplied. This further, supports our hypothesis in developing MNPs-based nanoformulations of anti-opioid agent.

\subsubsection{Cytotoxicity of MNPs-bound CTOP:}

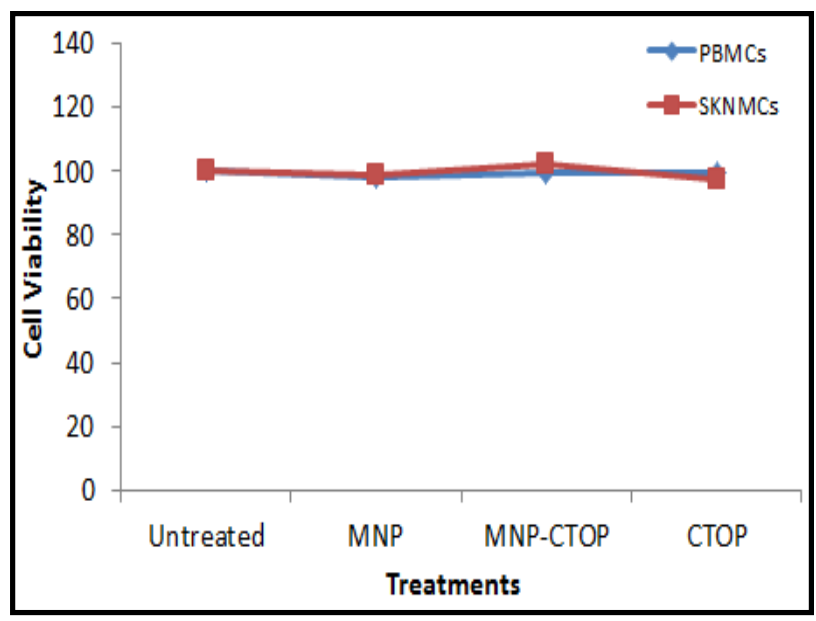

Figure 22: Percent cell viability of PBMCS and SK$\mathrm{N}-\mathrm{MC}$ cells $48 \mathrm{hrs}$ posttreatment with MNPs and MNP-CTOP nanoformulations.

Any exposure of external insults in the body, particularly when a sensitive organ such as brain is targeted, must be validated for its cytotoxic effect. It has been suggested that doses of MNPs within the permissible limit have nonsignificant safety concerns and are biodegradable (Jain et al., 2008). We 
examined the nonspecific cytotoxicity of MNPs with and without CTOP to PBMCs and SK-N-MCs. Our results showed that MNPs was neither cytotoxic to PBMCs nor to SK-N-MCs up to 48 hours of experimental treatments (Figure 22). The unaffected percent cell viability due to treatments of MNP with or without CTOP compared to untreated cells indicates their safe use as nanocarrier for drug deliver.

\subsection{BDNF adsorption on MNPs surface:}

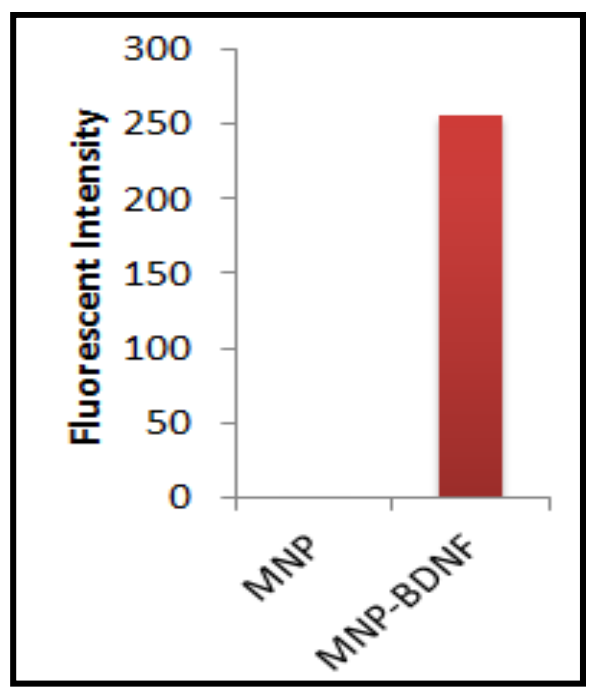

Figure 23: Fluorescent-based BDNF binding verification: Immobilization of red fluorescent dye tagged BDNF on MNPs emits fluorescence while MNPs without BDNF shows no fluorescence.

BDNF absorption on the surface of MNPs was determined using fluorescent-based detection method. As explained for the CTOP tagging, the Alexa flour 610 succinimidyl (NHS) esters were used for tagging of this protein. Fluorescent tagging is mediated by amide boding between dye and amine-containing ( $\mathrm{R}-\mathrm{NH} 2)$ moiety on proteins. This stable tagging provides could be used for many purposes such as quantification, imaging, etc. Significant 
fluorescent intensity was detected on the MNPs which were immobilized with dye tagged BDNF (Figure 23). Simultaneously, dye-exposed or non-exposed MNPs showed no trace of fluorescent activity. This suggests that BDNF could successfully be immobilized on the surface of MNPs.

\subsubsection{Time kinetics and binding isotherm of BDNF to magnetic nanoparticles:}

Several in-vitro and in-vivo studies demonstrate magnetically guided drug targeting (Alexiou et al., 2006; Lubbe et al., 1996). Here we characterize binding isotherm and kinetics of BDNF on MNPs, which is essential for determination of drugs sustainability on carrier and their safe dosing and bioavailability as well. The nanoformulation of MNP carrying BDNF was prepared by using different ratios of MNP and BDNF (1:0.05, 1:0.01, 1:0.015, 1:0.02, 1:0.025, 1:0.03, 1:0.35) in TE buffer (pH 7.4).

\section{Binding isotherm of BDNF on MNP}

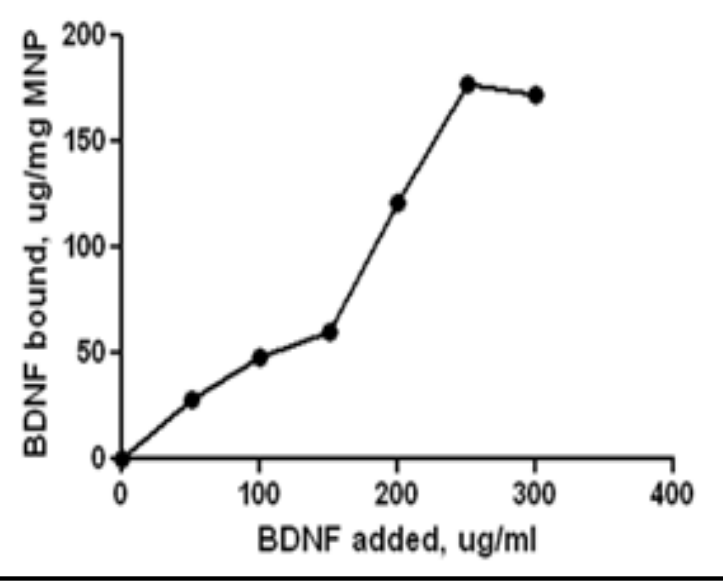

Figure 24: Binding isotherm of BDNF on MNPs: Ratios of 1:0.05, $1: 0.01,1: 0.015,1: 0.02,1: 0.025$, 1:0.03, and 1:0.35 of MNP and BDNF were incubated for $3 \mathrm{hrs}$ binding. Binding efficiency ( $\mu \mathrm{g}$ $\mathrm{BDNF} / \mathrm{mg} \mathrm{MNP}$ ) was measured by BDNF ELISA.

The BDNF ELISA was performed to calculate the binding efficiency ( $\mu \mathrm{g}$ BDNF /mg of magnetic nanoparticles) at different MNPs to BDNFs ratios. MNPs 
bound BDNF were separated from the binding reaction mixture using magnetic force and unbound fraction of BDNF present in supernatant was quantified. The difference between the total BDNF added and unbound BDNF was used to calculate the binding isotherm of MNPs-BDNF binding. As shown in figure24, a

maximum binding efficiency of $177 \mu \mathrm{g}$ of BDNF per mg of MNP is achieved in 3 hours of binding-reaction time. This corresponds to approximately $70 \%$ immobilization efficiency of BDNF on MNPs, suggesting an efficient and effective binding. Thus, similar to use of CTOP-magnetic nanoformulations, BDNFnanoformulations are also in accordance to our hypothesis and could be used for targeted brain delivery under the non-invasive magnetic influence.

\subsection{Apoptosis inhibition efficiency of MNPs bound BDNF in leukocytes:}

The mechanism of programmed cell death in apoptosis is triggered by a variety of internal and external stimuli. It could lead to sever pathological features such as those defined in case of certain inflammatory diseases of the brain and CNS infections (Kaul et al., 2001). Opioids are known to induce apoptosis in all types of cells including lymphocytes and neuronal cells (Nair et al, 1997; Yin et al., 1999; Singhal et al., 1998; goswami et al., 1998; Singhal et al., 1999; Freier and Fuchs, 1993; Fuchs and Pruett, 1993), mainly through the caspase-3 activation. Thus, inhibitors of caspase- 3 activity can rescue cells from the apoptotic cycle (Xia et al., 2010). In particular, use of such factor in addition to $\mu$ opioid receptor antagonist could be of therapeutic benefit in treatment of opiate addiction. Neurotrophic factor, BDNF is a class of such apoptosis-inhibitors which 
acts by blocking caspase-3 activity. As such, apoptosis-inhibition efficacy of MNP-BDNF nanoformulations was evaluated. Here again, morphine was used as agent for the apoptosis induction in lymphocytes.
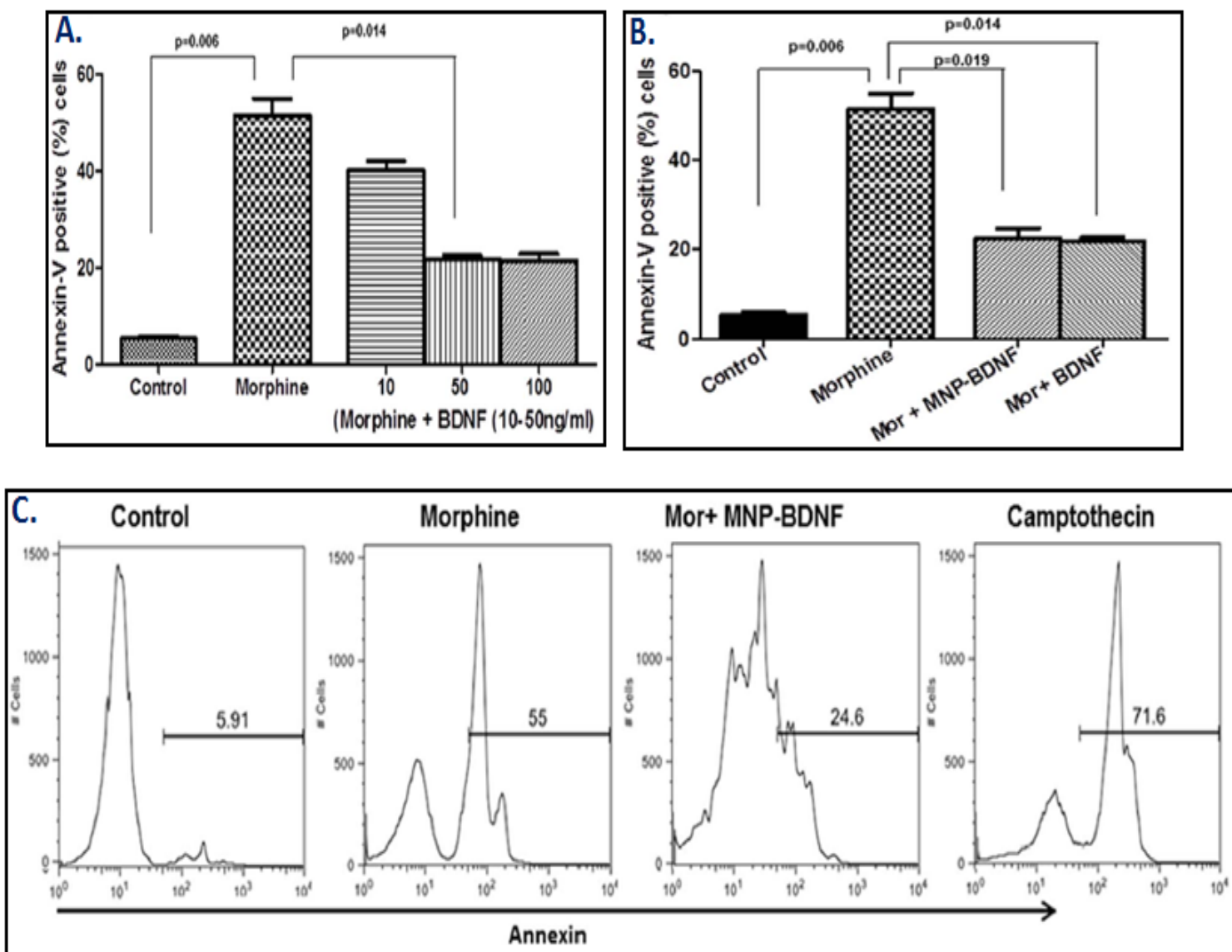

Figure 25: Flow-cytometry to evaluate the efficacy of MNPs bound BDNF on morphine induced apoptosis in PBMCs: (A). \% Annexin-v positive cells showing apoptosis-inhibition effect of different concentration of BDNF upon morphine treatment. (B). \% Annexin-v positive cells showing apoptosis-inhibition effect of MNPs- BDNF nanoformulations upon morphine treatment. (C). Representative histogram showing Annexin-v expression upon different treatments. Camptothecin is an established apoptosis inducer and serves as positive control.

To check whether BDNF activity is retained after immobilization by direct binding to MNP, we evaluated and compared the efficiency of MNPs bound 
BDNF to that with free BDNF (Figure 25). As expected, morphine treatment significantly $\left(10^{-7} \mathrm{M}\right)$ induced apoptosis in PBMCs $(55 \%)$ in compare with untreated cells $(p=0.006)$ (Figure 25A). We found that pre-treatment of BDNF could prevent the morphine-induced apoptosis in dose dependent manner. The optimum response was achieved at $50 \mathrm{ng} / \mathrm{ml}(p=0.019)$ and thus, same concentration was used for our further experimentation. Our results confirmed that morphine induces apoptosis in PBMC, and this effect was reversed by free BDNF. Similarly, morphine-induced apoptosis was also reversed upon treatment with MNP-BDNF nanoformulations ( $p=0.014$; figure 25B and 25C). Cells were also treated with camptothecin which serves as positive control for apoptosis induction (figure 25C). This suggests that binding of MNP did not interfere with the enzymatic activity of BDNF. These results showed that BDNF efficiently bound to MNPs and that bound form is as effective as that of free unbound BDNF in suppressing the apoptosis induced by morphine.

\subsection{Efficacy of MNPs bound BDNF in Astrocytes:}

\subsubsection{Modulation of CREB expression in Astrocytes:}

CREB play vital role in many neuronal-based responses such as memory and amnesia (Bourtchuladze et al., 1994; Bernabeu et al., 1997; Taubenfeld et al., 1999; Cammarota et al., 2000; Viola et al., 2000). Increased CREB phosphorylation has been shown to involve in memory consolidation (Jie luo et al., 2013).CREB mediated activities also functions as connecting bridge between opioid-induce secondary messenger systems and consequent alterations in gene expression 
during opioid exposure. As such, decreased phosphorylation of CREB has been noticed due to morphine-induced stimulation of opioid receptor (Mahajan et al., 2005). In this context, BNDF could exert beneficial therapeutic molecules because it assist in long-term memory formation, mainly via the activation of CREB in a time-dependent manner (Kwon et al., 2011; Alonso et al., 2005). Therefore, study was carried out to evaluate the ability of MNP-BDNF to modulate the morphine induced CREB expression in human brain astrocytes.
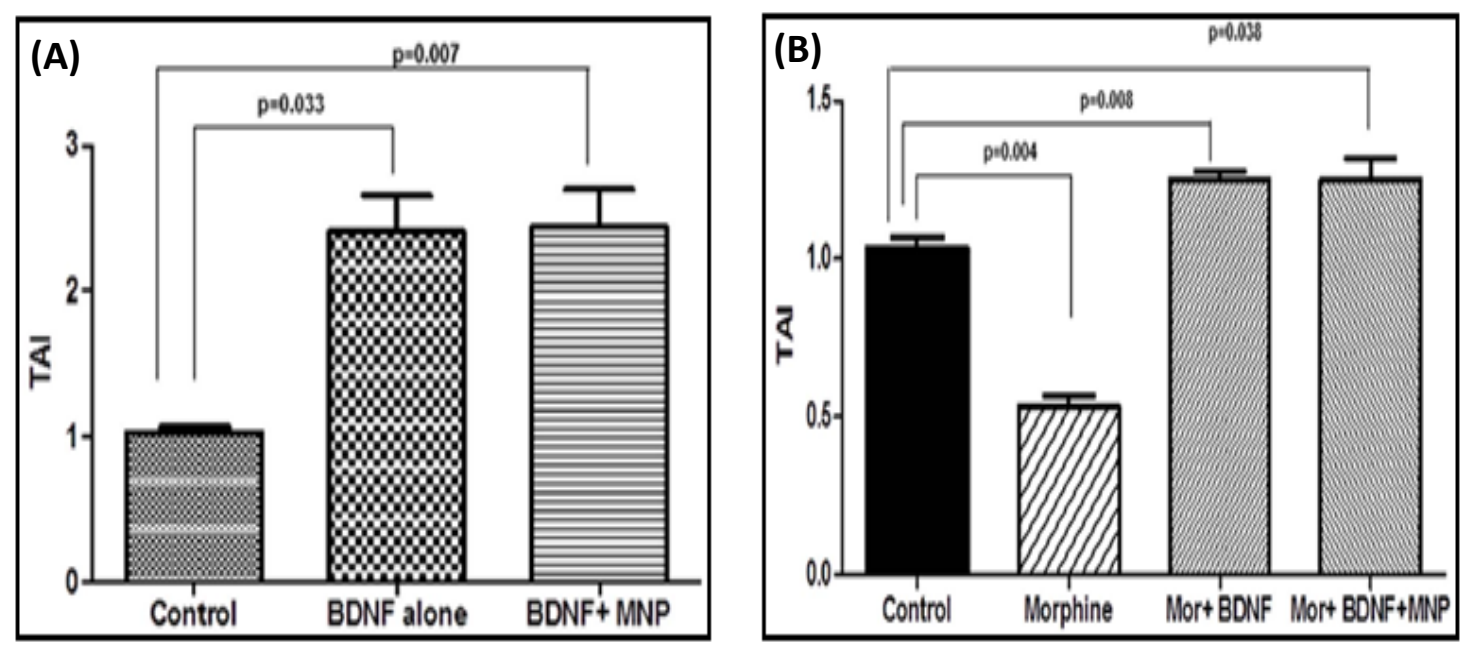

Figure 26: Trans-accumulation index (TAI) showing CREB expression: (A). TAl showing effect of Free BDNF and MNPs-BDNF nanoformulations on CREB expression. CREB expression is significantly upregulated either upon free or MNPs bound CTOP treatments. (B). TAI showing effect of Free BDNF and MNPs-BDNF nanoformulations on morphine mediated inhibition of CREB expression.

As shown in figure $26 \mathrm{~A}$, treatments of BNDF significantly upregulates CREB expression $(\mathrm{TAl}=2.4 \pm 0.25, \mathrm{p}=0.033)$. Similarly, MNPs bound BDNF resulted in a TAI value of TAl $=2.43 \pm 0.27(p=0.007)$. Thus, these comparable CREB expressions support our hypothesis that, irrespective of nanoformulations use for peripheral or neuronal cells, free and MNPs bound BDNF possess equal 
efficacy. Further, BDNF nanoformulations were tested for its ability to suppress the morphine-induced effect in astrocytes. As expected, in consistent with previous reports (Mahajan et al., 2005), morphine resulted in significant downregulation of CREB (TAl=0.533 $\pm 0.03 ; p=0.0004$; Figure 26B). When morphine exposed cells were pre-treated either with free or bound BDNF, downregulation of CREB expression was significantly reversed. Free and MNPsbound MNPs resulted in TAl values of $1.25 \pm 0.03(p=0.008)$ and $1.253 \pm 0.06$ $(p=0.03)$ respectively (Figure $26 B)$. This further suggests that nanoformulation could be used to alleviate morphine induced effects in CNS related problems.

\subsubsection{Inhibition of neuronal pathogenesis:}

Studies have shown that drug addiction alters the function of the neuronal circuit which includes changes in neuronal plasticity and synaptic transmitter release (Sarti et al., 2007; Frankfurt et al., 2011; Nestler, 2001). In the same line, morphine administration produces a persistent decrease in dendrite length and dendritic spine in neurons of different brain regions such as nucleus accumbens, visual cortex, sensory cortex, etc (Li et al., 2007; Robinson and Kolb, 1999). Here again, BDNF treatment has been reported to increase dendrite numbers (Bramham and Messaoudi, 2005; Chapleau et al, 2008). Therefore, we tested the ability of our nanoformulation to facilitate the neuroprotective efficacy in maintaining the dendrite spine density. Here also, morphine treatment resulted in decreased spine density $\left(0.055 \pm 0.02\right.$ per $\left.\mu m^{2} ; p<0.002\right)$ in compare to untreated groups (Figure 27A and 27B). This significant decrease in morphine-induced spinal density is prevented upon BDNF treatment. Both free and MNPs-bound 
BDNF showed equivalent efficiency in preventing the morphine induced spinal degeneration (Figure 27B).
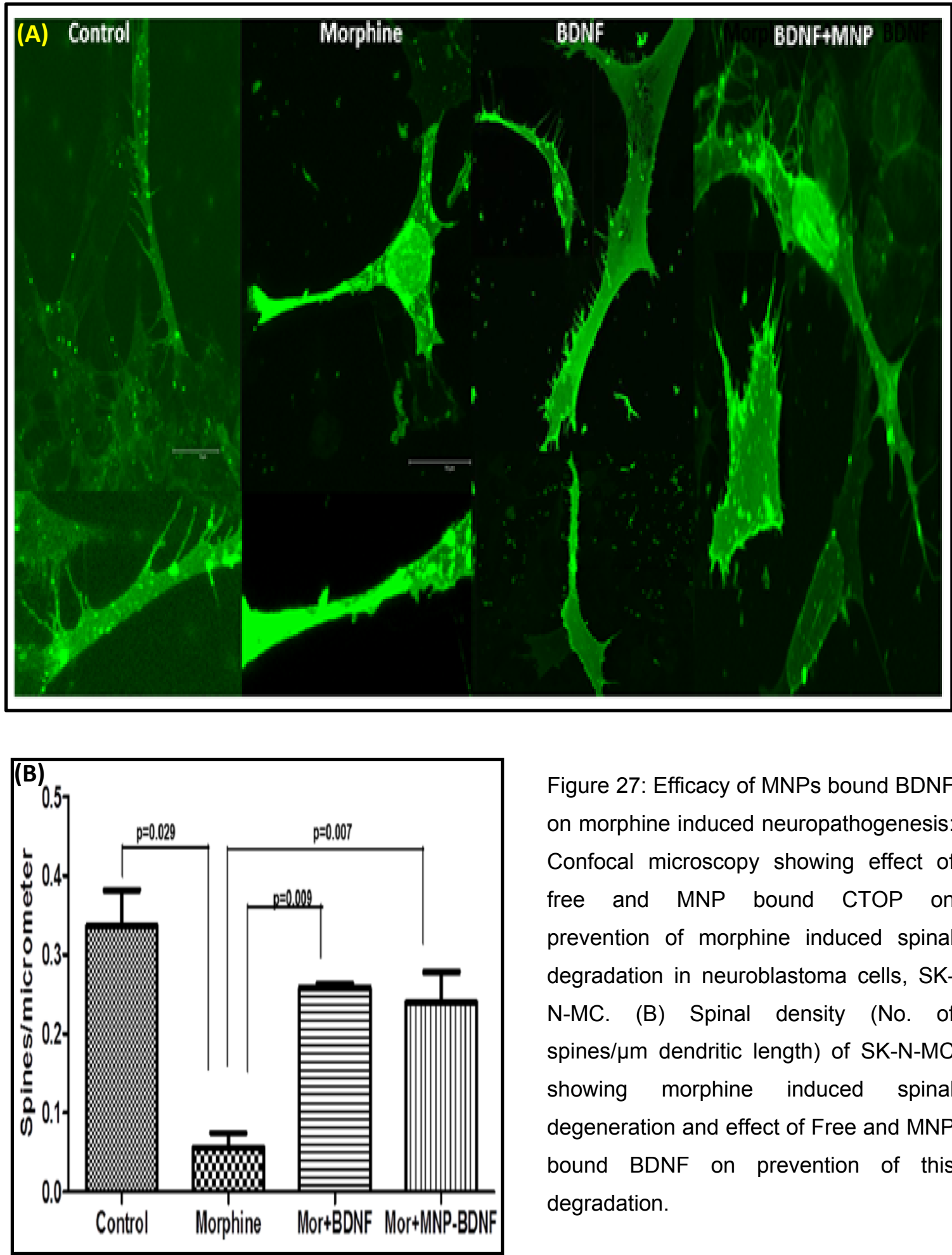

Figure 27: Efficacy of MNPs bound BDNF on morphine induced neuropathogenesis: Confocal microscopy showing effect of free and MNP bound CTOP on prevention of morphine induced spinal degradation in neuroblastoma cells, SKN-MC. (B) Spinal density (No. of spines/ $/ \mathrm{m}$ dendritic length) of SK-N-MC showing morphine induced spinal degeneration and effect of Free and MNP bound BDNF on prevention of this degradation. 
Pre-treatment of MNPs bound BDNF resulted in an average spine density of $0.258 \pm 0.09$ per $\mu m^{2}(p=0.007)$. Our result suggests that, similar to their efficacy in suppressing morphine-induced apoptosis in PBMCs, BDNF nanoformulations possesses comparable effect to that of free BDNF in preserving the neuropathogenesis.

\subsection{Cytotoxicity of MNPs-bound BDNF:}

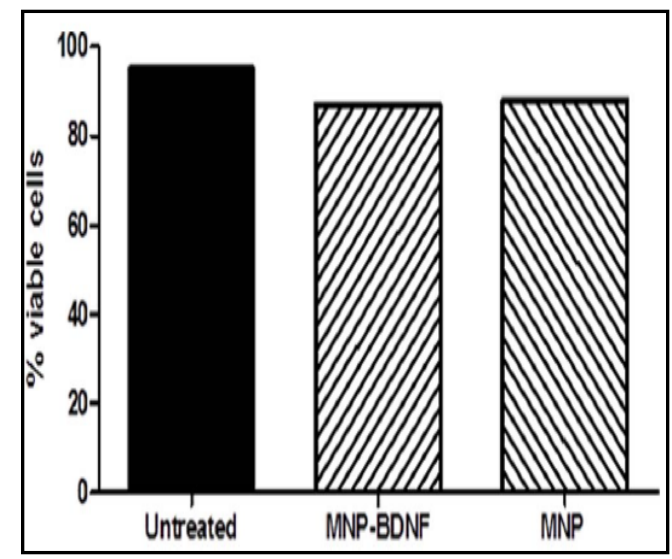

Figure 28: Percent cell viability of PBMCS 48 hrs post-treatment with MNPs and MNP-BDNF nanoformulations.

One of the major concerns while using nanomaterials in medicine is that of potential toxicity. As such, evaluation of cell viability is important for the nanoparticle application in medicine. We examined the nonspecific cytotoxicity of MNPs with and without BDNF to PBMCs. Our results showed that MNPs possess insignificant toxicity for PBMCs. Approximately 95\% cells were found live (Figure 28), in both, MNPs and MNPs-BDNF treatments. This unaffected percent cell viability suggests their safe use as nanocarrier for drug deliver. In the same line, based upon in vivo studies, it has been suggested that doses of MNPs within the permissible limit have non-significant safety concerns and are biodegradable (Jain et al., 2008). 


\subsection{Characterization of MNPs-based liposomal nanocarriers:}

Magnetite $\left(\mathrm{Fe}_{3} \mathrm{O}_{4}\right)$ is the most commonly used magnetic nanoparticles in the field of biomedicine, mainly due to its biocompatibility. In accord with the basics of nanotechnology, MNPs of different sizes, ranging from a few up to tens of nanometers, have been extensively investigated for disease diagnosis and target-specific improved drug delivery. Generally drugs are either directly immobilized on the MNPs surface or tethered via coating of organic/inorganic surfactants such as PEG. In either case, attached drugs are exposed to external environment and possess threat of rapid decomposition due to metabolic (enzymztic mainly) activity of peripheral circulation (blood) before it could reach to target. Thus, an approach to protect drugs from exterior must be devised for advancement of MNPs-based drug delivery. Recently, a hybridization strategy where MNPs is encapsulated in liposomes termed "Magneto-liposome (ML)", has emerged as a possible solution.

\subsubsection{Characterization of ultrasmall magnetic nanoparticles:}

Potential application of ML-based drug delivery across blood-brain barrier (BBB), in CNS, is very limited. In the wake of CNS sophisticacy, an ideal drug delivery carrier should co-incorporate maximum drug bioavailability with minimum waste constituent. One way this could be achieved is by increasing the encapsulation potential of liposomes with no affect on overall ML size. Higher loading density of MNPs in liposomes would require synthesis of smallest particles in the nanometer range suitable for the targetting and drug- 
bioavailablity. Particles of $>10 \mathrm{~nm}$ can sustain in the the systemic circulation; however, that of lesser size lost due to permeability of vascular endothelium and prohibits drugs to reach target-site.

There are several methods to synthesize $\mathrm{Fe}_{3} \mathrm{O}_{4}$ nanoparticles such as coprecipitation, microemulsion, high temperature decomposition, oxidation of magnetite, etc. (Sun et al., 2004; Frascione et al., 2012; Jayapaul et al., 2011; ). Co-precipitation is regarded as one of most efficient, circumstance friendly and cost-effective way to prepare MNPs on nanoscale. This method mainly uses two different approaches to reduce ferrous ion from $\mathrm{FeCl}_{3}$ - either by $\mathrm{Na}_{2} \mathrm{SO}_{3}$ or $\mathrm{FeSO}_{4}$. The later approach results in formation of rod shaped nanoparticles of over $30 \mathrm{~nm}$. Liposomal encapsulation of particles of this shape and size will have a greater impact on the overall size of MLs and may not be suitable for delivery across many physiological barriers such as BBB, stomach epithelial, etc. Most importantly, it may significantly downgrade the colloidal stability of MLs in the peripheral circulation. The $\mathrm{Na}_{2} \mathrm{SO}_{3}$-based reduction approach possesses advantage in producing round MNPs with smaller size, probably due to the gentle reduction ability from $\mathrm{Na}_{2} \mathrm{SO}_{3}$ in aqueous medium. Maghemite $\left(\mathrm{Fe}_{2} \mathrm{O}_{3}\right)$ is the primary product of this reduction reaction which is further oxidized under acidic condition resulting in $\mathrm{Fe}_{3} \mathrm{O}_{4}$ nanoparticles. As determined by TEM, ultrasmall magnetite nanoparticles of 7-10 nm possessing excellent dispersion property in aqueous medium could be synthesized by this approach (Figure. 29A). The nano-sized particles possess remarkably higher specific surface area which improves drug loading ability and dissolution rate influencing the bioavailability. 
These particles could also manipulate and target at the subcellular organelles levels. The crystal structure of synthesized magnetite particles was confirmed by X-ray diffraction spectroscopic measurement (Figure 29B). The X-ray spectrum consists of magnetite-specific peaks which correspond to $220,311,400,511$, and 440 planes.

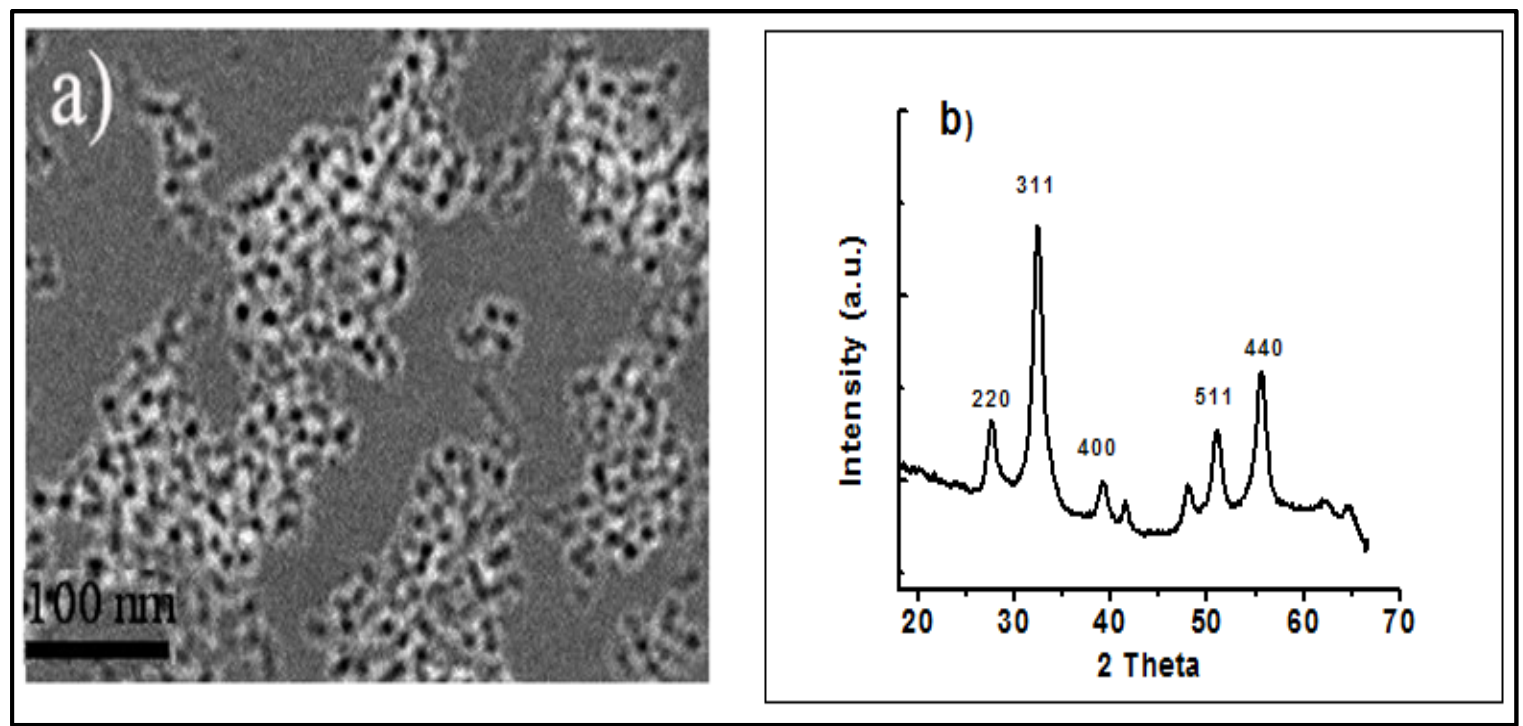

Figure 29: Characterization of ultrasmall magnetite nanoparticles: a) TEM image showing MNPs of 7-10 nm. b) XRD spectrum showing magnetite-specific characteristics plane.

Magnetic hysteresis loops for these particles, which displayed strong magnetic property, were measured between +1200 to -1200 Oersted (Oe). As shown in figure 30A, the nanoparticles exhibit a superparamagnetic behavior with no coercivity and remanence at room temperature. The superparamagnetism can be utilized for simultaneous monitoring and quantitation of MNPs distribution specific or nonspecific to various tissues. Thus, quantitation of localization of MNPs associated drugs could be possible using techniques like magnetic resonance imaging (MRI) and magnetometery due to variation in the surface 
charge of naked and drug-bound nanoparticles leading a way for determining the site-specific optimal or suboptimal drug-dosing. Distribution of charge on the surface of synthesized MNPs was determined by measuring the zeta-potential at different $\mathrm{pH}$ level of dispersion solution (Figure $30 \mathrm{~b}$ ). Interestingly, with $\mathrm{pH}$ values changing from acidic to basic, zeta potential of MNPs alternated from positive charge of $+26 \mathrm{mV}$ at $\mathrm{pH} 4.75$ to negative charge of $-23 \mathrm{mV}$ at $\mathrm{pH}$ 8.5. The isoelectric point of MNPs was determined at $\sim 7.1 \mathrm{pH}$. We noticed that content of dispersion solution also affects the surface charge of MNPs. As such, in TrisEDTA buffer ( $\mathrm{pH} 7.4)$, MNPs displayed zeta potential of approximately $-21 \mathrm{mV}$ which is nearly equivalent to that obtained in $\mathrm{H}_{2} \mathrm{O}$ with $\mathrm{pH} 8.5(-23 \mathrm{mV})$.



Figure 30: a) Magnetic hysteresis loop of MNPs showing no coercivity and remanence at room temperature suggests its superparamagnetic behavior. b) Surface charge of MNPs varies according to $\mathrm{pH}$ values of suspension solution.

Charge on the surface of $\mathrm{Fe}_{3} \mathrm{O}_{4}$ particles is developed due to its amphoteric property in aqueous media. Acting as Lewis acid, at the hydrated solid/water interface, magnetite adsorb/coordinate water or hydroxyl group and 
gets $\mathrm{H}+/ \mathrm{OH}$ - ions along it surface. These ions can be replaced by other organic or organic anion, form hydrogen bond, and adsorbs proton or cations. This could allow direct immobilization of various biomolecules/molecules on MNPs surface via hydrogen bonding, hydrophobic interaction, and electrostatic repulsion (Peng et al., 2004; Yu et al., 2013). Also, the surface charge of magnetic nanoparticles could be converted either to positive or negative with different kinds of coating such as, the polyelectrolyte coating, silica coating, etc. (Ding et al., 2007; Gittins et al., 2001; Chen et al., 2008).

\subsubsection{Characterization of $M L$ nanocarriers:}

As stated earlier, greatest challenge towards the successful application of MNPs in drug delivery is protection of associated drugs from enzymztic decomposition of blood circulation. Naked MNPs also interact with various plasma/serum proteins which could significantly affect the potential outcome of its applications in other drug-related and unrelated biological uses such as target specificity, MR imaging, etc. Although liposomal encapsulation of MNPs is looked upon as potential solution of these concerns, physiological integrity and stability of magneto-liposomal colloids needed to be addressed for their effective manipulation. Additionally, ways to maximize the target-reachability must be incorporated in the nano-formulated carrier. It has been suggested that modifications such as PEGlyation of liposomal surface could improve the inherent poor stability of conventional liposomes. Also, liposomal surface can be engineered for active targeting by applying surface charge modifications and/or 
conjugation of antibodies/ligands specific to cells or tissues. Here, we used PEGylated lipid, DSPE-PEG, for liposome formulation. The PEG in this lipid is tethered to the hydrophilic head of phospholipid bilayer; thus, upon liposomal formulation PEG will be extended outside from the surface. In addition to provide colloidal stability, PEGylation prevents liposome-induced immunogenicity and could also reduce their uptake by reticuloendothelial system resulting in improved plasma circulation time and increased bioavailability to reach the target (Suri et al., 2007; Peng et al., 2012).

To maximize the reachability of nanocarrier to the target i.e. in this case transportation across BBB, we embedded transferrin, a ligand for the HBMVECs specific transferrin receptor, on the surface of PEGylated ML. Presence of MNPs and BBB specific receptor's ligand on the same carrier will synergize the transmigration across BBB. While MNPs will influence the movement in the brain under external magnetic force, presence of ligand will add to this effect by providing uptake-specificity for BBB cells (in this case for HBMVECs). The ML possessing such dual targeting mechanisms could be epitomized for many other target-oriented deliveries. We further expanded the multifunctionality of ML by making it fluorescent. This was achieved by adding green fluorescent tagged phosphatidylethanolamine, namely CFPE, in the liposomal formulation mixture. Fluorescent addition to ML could serve as a tool for nanocarrier associated pharmacokinetics study such as quantification of cellular uptake or entrapment, tracking localization in tissues, etc. More importantly, co-incorporation of fluorescent in the MNPs-based nanocarrier provides two different imaging 
options, MNPs-based imaging such as MRI and magnetometery and fluorescentbased imaging. We believe that easy availability of fluorescent-based imaging technique will widen the use of $M L$ as convenience and cost-effective tools in the targeted-drug delivery. Nonetheless, MNPs could always be available for targeted delivery and more powerful imaging, such as MRI, could be applied as per necessity.

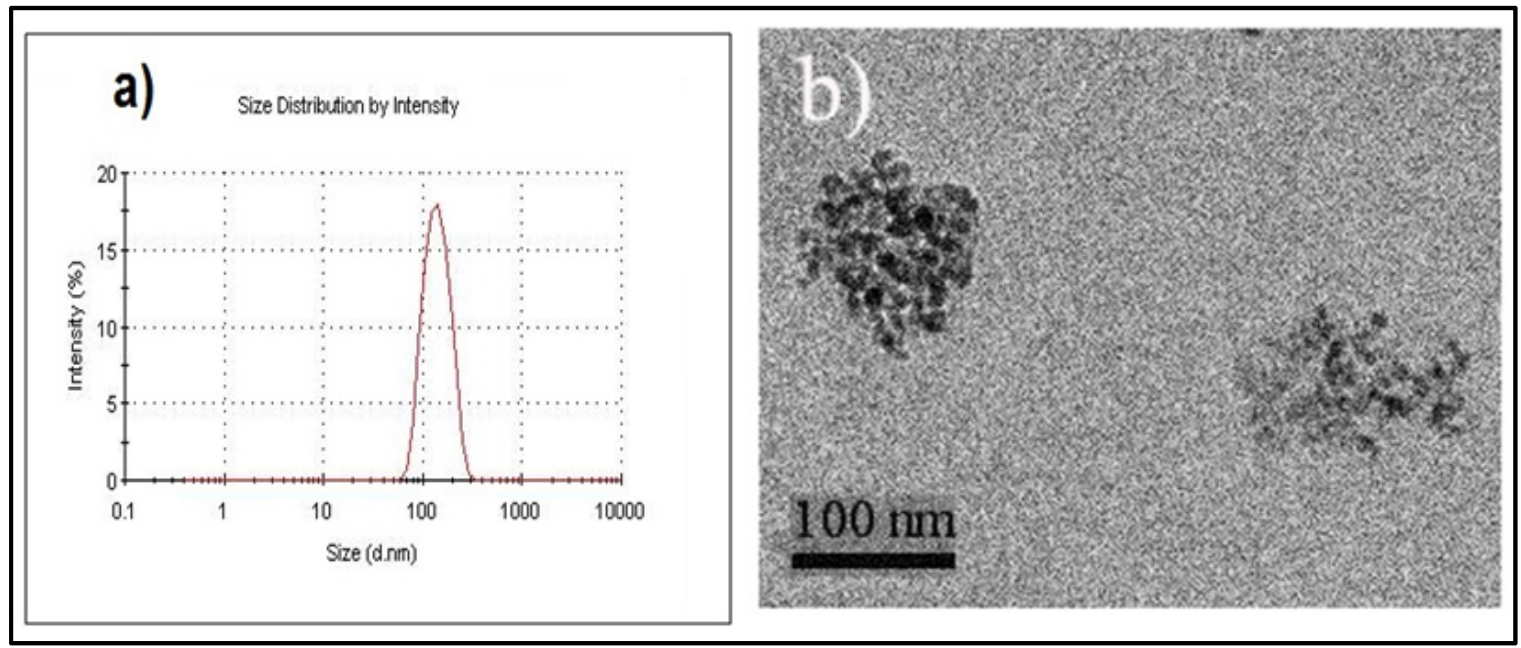

Figure 31: Characterization of magnetic-liposomes (MLs): a) Dynamic laser scattering (DLS) measurement of hydrodynamic size distribution of MLs. Average diameter size of this nanoformulation was approximately $130 \mathrm{~nm}$. b) TEM image of ML nanocarriers containing MNPs (black dots) supports the size of MLs as determined by DLS measurement.

Size of PEGylated magneto-liposomal nanocarriers formulated using extrusion technique was determined by DLS (Figure 31A) and further TEM was performed to verify this formulation (Figure 31B). As evident from both figures, the average diameter size of this nanoformulation was approximately $130 \mathrm{~nm}$. Encapsulation of MNPs into the liposomes have been proved to be difficult task 
and a maximum of $15 \%$ EE have been shown earlier (Deng et al., 2012). We here report EE of $60.0 \%$ which could possibly be attributed to the ultrasmall sized MNPs. In the same line, TEM image shows that the ML formulation is filled with ultrasmall MNPs. Nonetheless, EE of ultrasmall MNPs in liposome can be manipulated by changing the ratio of particles with liposomes during the formulation process. Higher MNPs content may significantly improve the contrast enhancement effect of MRI. Larger sized MNPs have been previously used due to their MR contrast enhancement effect (Qiao et al., 2012; Jun et al., 2005); however, in view of transportability across BBB, smaller MNPs as small as $10 \mathrm{~nm}$ may provide better pliantness to the liposomes which, in addition to their use as MRI, could remarkably influence its transendothelial extravasation under external non-invasive magnetic influence. More importantly, higher EE i.e. more no of MNPs in a liposome will provide higher per unit loading surface area resulting in significantly higher per unit drug loading efficiency of $\mathrm{ML}$ nanocarriers. Furthermore, it is challenging to maintain the mono-dispersibility of the nanocarriers. It becomes even more important in view of their potential application in targeted drug delivery. Although PEGylation add to the colloidal stability and assist in maintaining the liposomal mono-dispersion, incubation temperature during the formulation process plays a vital role. We achieved highly mono-dispersed liposomes using an incubation- and extrusion- temperature of $\sim 40^{\circ} \mathrm{C}$ which is higher than the thermogravimetric analysis points for all lipids. Homogenous size of ML nanocarriers was attained by using polycarbonate filter membrane of different pore-size $(400 \mathrm{~nm} / 200 \mathrm{~nm} / 100 \mathrm{~nm})$ during the extrusion 
process. Notably, molar concentration ratio of DSPE-PEG used during the formulation process was controlled within $10 \%$, a critical micelles concentration limit above which PEG influence the micelles formation. The formulated ML was subjected to DLS for measurement of zeta potential which showed near neutral charge of $-0.8 \pm 0.2 \mathrm{mV}$ on the surface of this nanocarrier. Thus, possibility of charge-mediated cellular uptake of $\mathrm{ML}$ will be minimized in the peripheral circulation i.e. external magnetic force will remain the only controlling force of this nanocarrier for effective movement up to the target area. In order to provide more established BBB specificity to this nanocarrier, transferrin was conjugated on its surface. Transferrin is one of rare proteins which have free access across the intact BBB and carry essential nutrients into the brain. Transferrin conjugation on ML surface was confirmed by spectrophotometry (Figure 32).

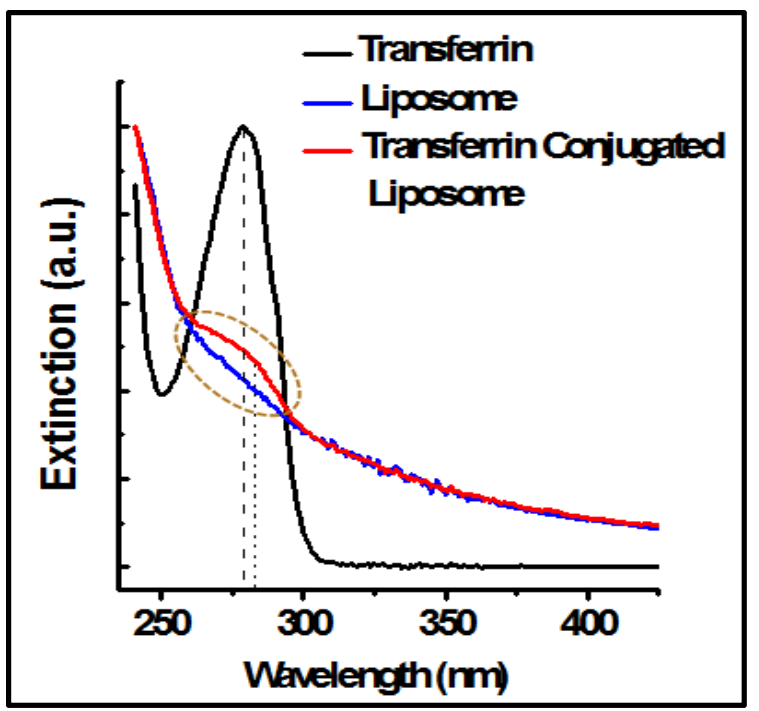

Figure 32: Characterization of transferrin conjugation to MLs: spectra of transferrin conjugated MLs shows rise of the optical density peak in the transferrin-specific wavelength range.

Similar to the previous study of $\mathrm{Xu}$ et al (2008), spectra of transferrin conjugated magnetic-liposomes showed rise of the optical density peak in the transferrin-specific wavelength range. It should be noted that inclusion of less 
than $10 \%$ of PEG in formulation mixture results in homogenous PEGylation rather than forming thick corona which was noticed in the work of Gao et al (2006). The shielding effect of thick PEG corona may cause improper ligand bind and also may prevent proper interaction of ligand to the receptor. In other hand, the homogenous embedding of 2000 Da PEG on the ML surface may have negligible or zero interference in the interaction of $80 \mathrm{kDa}$ transferrin and its cellular receptors. Similarly, homogenous transferrin distribution should not affect the original objective of PEG in providing colloidal stability. As determined by DLS, Transferrin conjugation to the ML surface neither has significant effect on its hydrodynamic size nor did it affect the surface charge distribution.

\subsubsection{Physiological sustainability of $M L$ nanocarriers:}

The formulated ML nanocarriers were assessed for its sustainability in the physiological equivalent temperature and circulation. Nanocarriers suspended in the PBS were incubated in $37^{\circ} \mathrm{C}$ for up to 30 hours and their size was measured at different time points using the DLS. As shown is figure $33 \mathrm{~A}$, size of these formulations remained unchanged. Similarly, fluorescent intensity emitted from equal amount of ML carriers was constant through-out the incubation period (Figure 33A). It should be noted that fluorescent integration in $M L$ improves its multifunctionality such as easy manageable quantification and visualization of carriers during experimental settings. This was achieved by adding green fluorescent tagged lipid, namely CFPE, in the liposomal formulation mixture. Sustainability of these carriers was further evaluated in the in vitro closed 
circulation system which consists of a bidirectional, self-priming peristaltic capillary pump. A schematic of this pump is shown in figure 33B. Here also, both, the structural integrity and fluorescent intensity of $M L$ nanocarriers remained unaffected through 120 equivalent blood-circulations of experimental settings (Figure 33D). Fluorescent intensity study together with the DLS measurement shows that the formulated ML nanocarriers could sustain its structural integrity in the simulated blood circulation and physiological temperature for considerable amount of time. This suggests toward the possible use of fluorescent ML nanocarriers for in vivo drug delivery and optical imaging. Nanocarriers were also looked for their storage durability at $4^{\circ} \mathrm{C}$ for around one month. Again, constant diameters were determined throughout the storage period (Figure 33C). Also, as expected, hydrodynamic difference between the diameter of transferrin conjugated or unconjugated liposomes remained less than $10 \%$ throughout the storage time. Nonetheless, it is worth mentioning that the colloidal stability and optical properties of the ML nanocarriers remained unaffected due Transferrin conjugation. This suggests that these ML nanocarriers possess longer storage stability and drugs loaded on these carriers could have minimum leaching effect. In fact, consistent florescent intensity either during exposure of physiological temperature, peristaltic circulation or storage implies towards minimum leakage from formulated ML nanocarriers. Larger size MNPs $(35 \mathrm{~nm})$ were also tested for encapsulation in the liposomes. However, stability and precipitation remained an issue because ML could sustain in suspension for not more than couple of hours. 

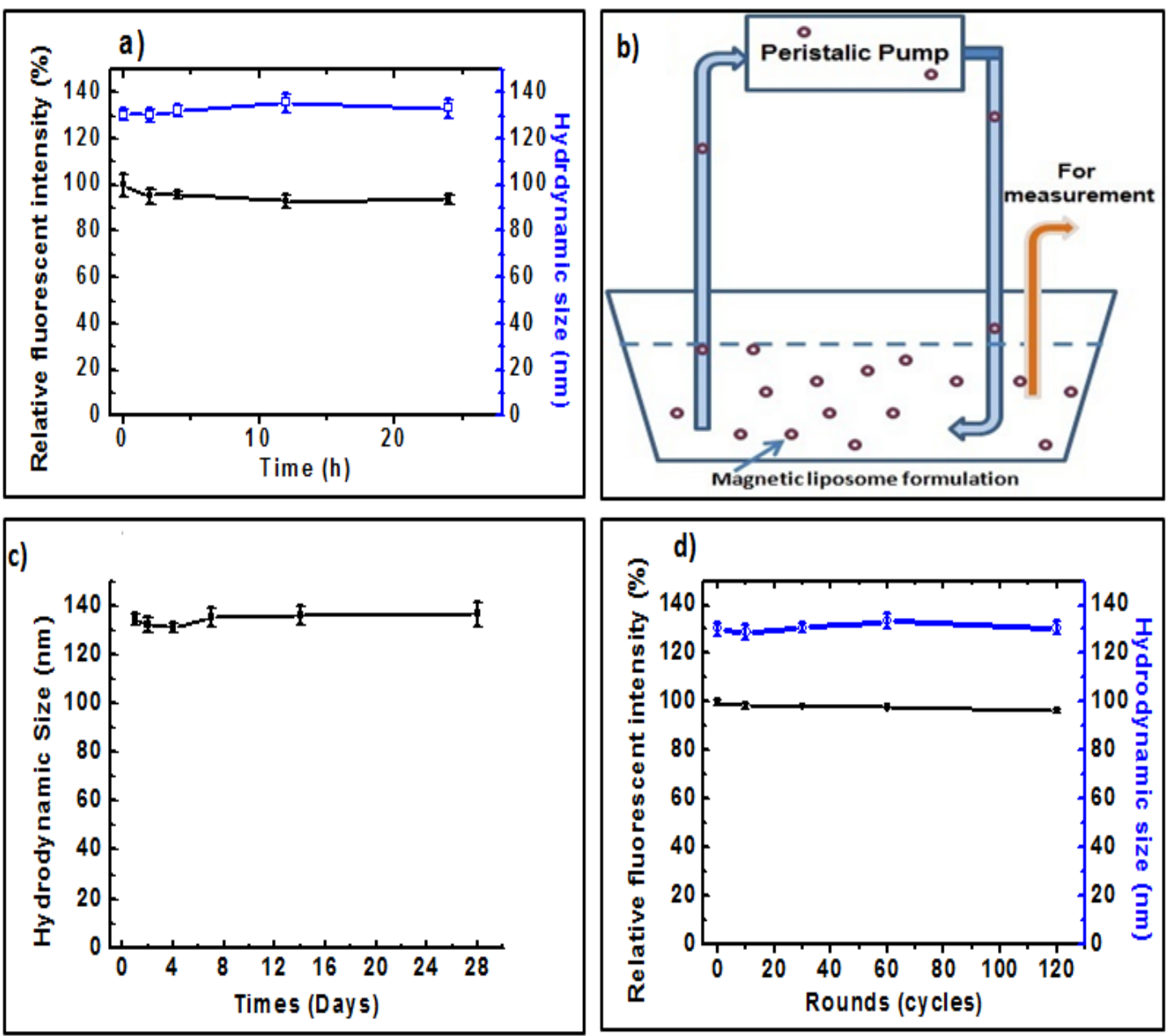

Figure 33: Characterization of MLs sustainability: a) Florescent intensity (black) and average diameter (Blue) of $\mathrm{ML}$ nanoformulations incubated at $37^{\circ} \mathrm{C}$. It suggests that structural integrity and fluorescent intensity of ML nanocarriers remained unaffected up to $30 \mathrm{hrs}$ of experimental period. b) Schematic of in vitro closed circulation system: The bidirectional, self-priming peristaltic capillary pump simulates physiological equivalent blood-circulation. c) Florescent intensity (black) and average diameter (Blue) of $\mathrm{ML}$ nanoformulations after various round of in-vitro circulations. It suggests that structural integrity and fluorescent intensity of ML nanocarriers remained unaffected up to 120 circulations of experimental period. d) Average diameter (Blue) of ML nanoformulations in storage at $4^{0} \mathrm{C}$ suggests its structural integrity up to 28 days of experimental duration. 


\subsection{Transmigration of $M L$ nanocarriers across $B B B$ :}

The BBB transmigrability of the ML nanocarriers was evaluated using an in vitro human BBB model. As described previously by Gandhi et al. (2010), the BBB model was established in a bi-compartmentalized transwell where HBMECs and HAs were grown to confluency on the upper chamber and underside of lower chamber respectively. The intactness of grown BBB was determined by TEER values. A TEER value of $\sim 200 \mathrm{ohms} / \mathrm{cm}^{2}$ in compare to the control (non-cultured wells) is considered consistent with the formation of intact BBB. Following the TEER determination, fluorescent $M L$ nanocarriers were subjected to different wells either in presence or absence of external magnetic force. As shown in Table 3, initial TTER values of all treatment groups were close to standard 200 ohms $/ \mathrm{cm}^{2}$. As expected, significantly higher transmigration of $\mathrm{ML}$ nanocarriers were detected across BBB due to exposure of external magnetic force (Figure 34A and 34B). Incorporation of fluorescent in the ML nanocarrier was used for quantification of its transmigration from apical to basolateral layers in the BBB model. Transferrin conjugated ML nanocarriers in presence of external magnetic force (Force) showed higher transmigration than nanocarriers which lacks external force (No force). Approximately $2-3$ folds (100\% vs $30-50 \%$ ) increased transmigration was achieved and this continued till $16 \mathrm{hrs}$ of experimental period. It could possible that magnetic force may allow transient change in BBB permeability resulting in increased transendothelial extravasation of $\mathrm{ML}$ nanocarriers. Magnetic force induced increased BBB permeability has been reported earlier in few studies. Working with the $\mathrm{Fe}_{3} \mathrm{O}_{4}-\mathrm{PEG}$ nanocarriers, Qiao 
et al (2012) showed an alteration in BBB permeability after 4 hrs of exposure. Similarly, treatment of polysorbate derived nanoparticles resulted in sharp increase in permeability from 4-7 hrs and accordingly TEER values were reversed from less to high [Rempe et al., 2011].
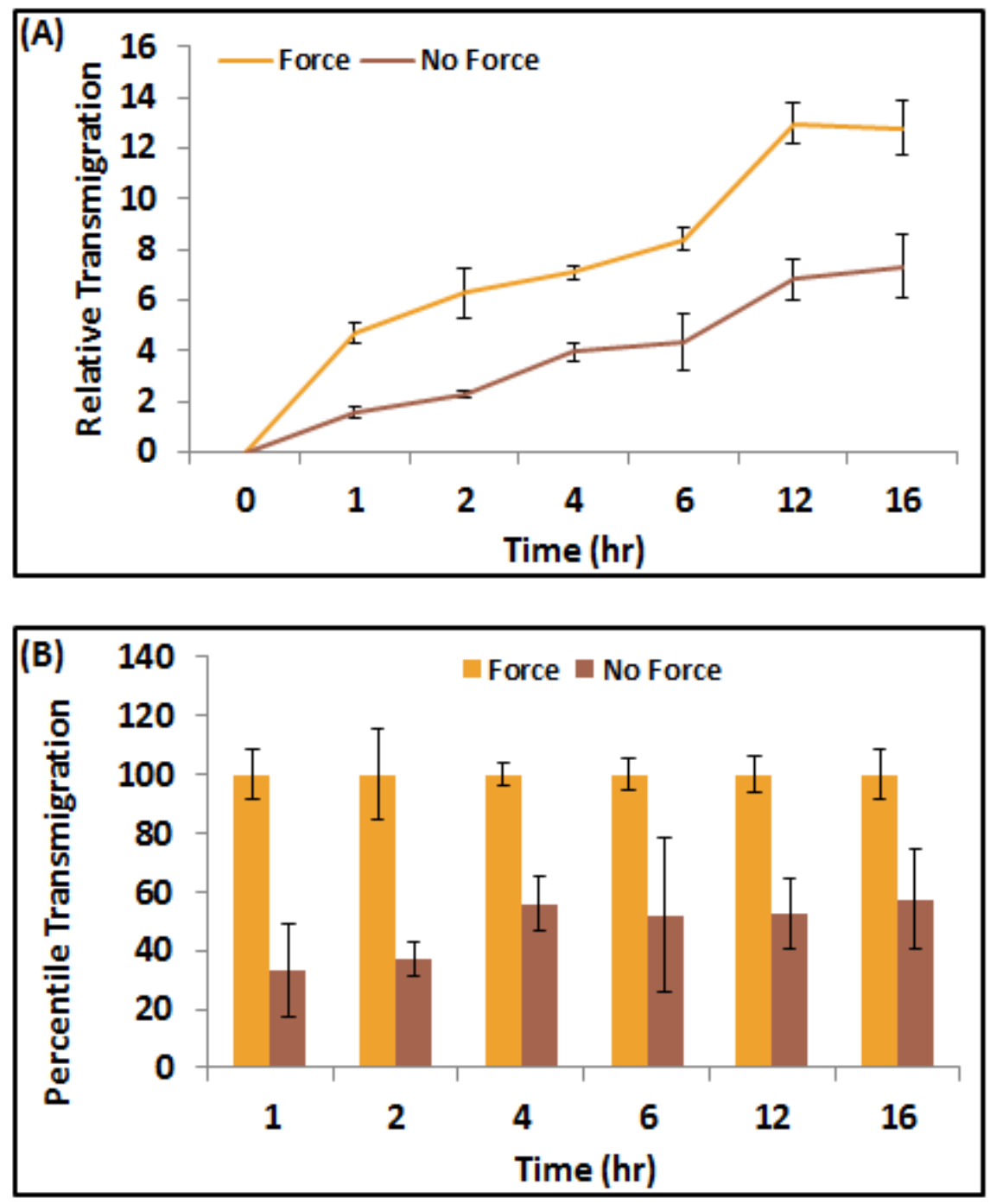

Figure 34: a) Relative transmigration of $\mathrm{ML}$ nanocarriers in the presence and absence of external magnetic force across in vitro BBB. b) Percentile transmigration of nanocarriers at different time. 
In our study, TEER values of BBB remain closer to the standard 200 ohms $/ \mathrm{cm}^{2}$ both at the beginning and end of experimental period, suggesting unaffected integrity of BBB due to exposure of different nanoformulations (Table 3).

\begin{tabular}{|c|c|c|c|}
\hline $\begin{array}{c}\text { TEER values } \\
\left(\mathrm{ohms} / \mathrm{cm}^{2}\right)\end{array}$ & Force & No Force & Untreated \\
\hline Before treatment & $189.5 \pm 7.5$ & $191.2 \pm 8.5$ & $192.2 \pm 9.5$ \\
\hline After treatment & $186.2 \pm 8.1$ & $187.8 \pm 8.4$ & $190.6 \pm 8.9$ \\
\hline
\end{tabular}

Table 3: Transendothelial electrical resistance (TEER) values of the in vitro BBB model before and after treatment ML nanocarriers in the presence and absence of external magnetic force

The transmigration pattern of nanocarriers was further verified by measuring the iron concentration. Triton mediated liposomes breaking results in release of encapsulated and/or uptaken $\mathrm{Fe}_{3} \mathrm{O}_{4}$ which is exposed to ammonium thiocyanate following oxidation via ammonium persulfate. Quantification of resulting iron-thiocyanate emits red color at $490 \mathrm{~nm}$ gives concentration of $\mathrm{Fe}_{3} \mathrm{O}_{4}$ uptaken by BBB cells. As evident from figure 35 , consistent with the transmigrability, higher iron concentration was found when external magnetic force was used. This supports our observation that external magnetic force may result in increased accumulation of ML nanocarriers at the BBB causing higher transendothelial extravasation and/or receptor-mediated transmigration. 


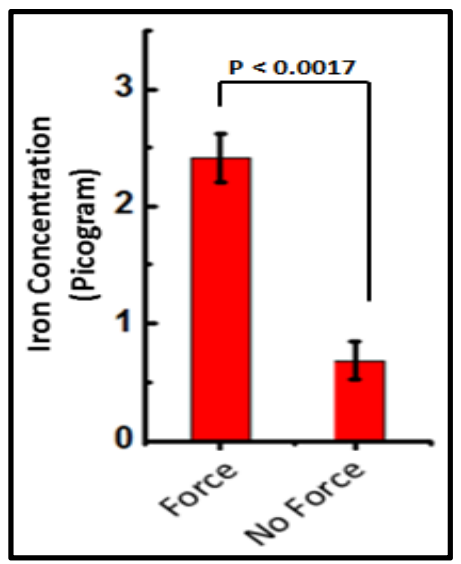

Figure 35: The $\mathrm{Fe}_{3} \mathrm{O}_{4}$ content in lower chamber post-transmigration.

\subsection{Cytotoxicity of ML nanocarriers:}

Percent viability of astrocytes and HBMVE cells remains unaffected due to treatments of our nanocarriers (Figure 36). In the same line it has been suggested that doses of MNPs within the permissible limit have non-significant safety concerns and are biodegradable (Jain et al., 2008). This suggests that ML nanoformulations possess required potential to be used as carrier for drug deliver across BBB.

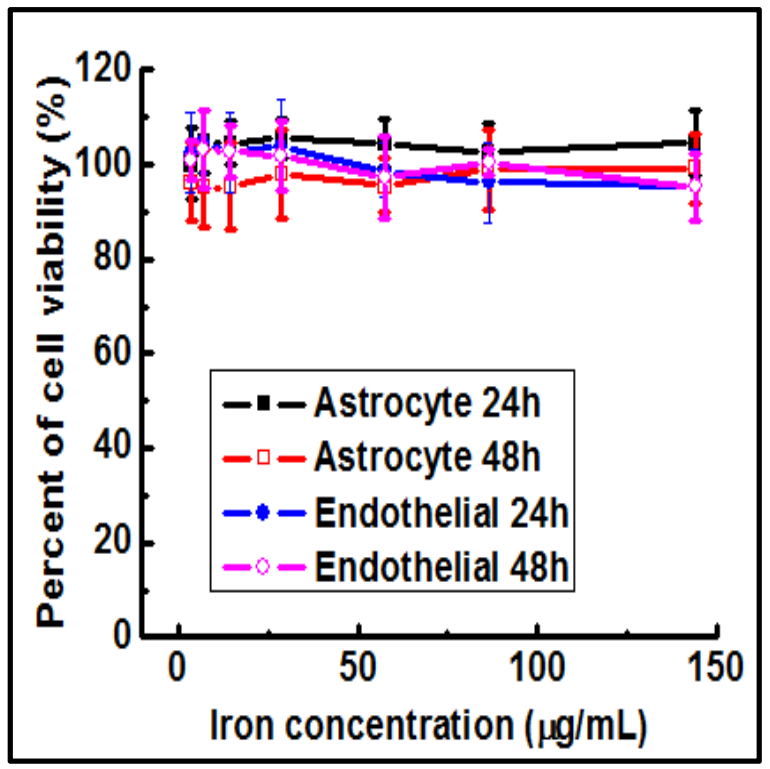

Figure 36: Percent cell viability of primary endothelial cells and astrocyte cells 24 and $48 \mathrm{hrs}$ post-treatment with different concentration of ML nanocarriers. 


\subsection{BBB transmigration and efficiency of BDNF nanoformulations:}

We evaluated the ability of MNPs-BDNF nanoformulations to cross the BBB. As stated previously, free or MNPs bound BDNF were added to the apical chamber of an established in vitro BBB model and transmigration was influenced by applying external magnetic force beneath the lower chamber. The result obtained by BDNF ELISA for the sample collected from the basal chamber show that approximately $73 \%$ of the MNP bound BDNF was able to transmigrate across BBB (Figure 37A). This corresponds to 3.5 folds higher transmigration than free BDNF. Our results suggest that transmigration ability of BDNF across BBB increased significantly when MNPs were used as carriers. Again, we verified the integrity of BBB and as demonstrated previously, the BBB remained undamaged. A TEER reading of 280.4 and 276.5 were obtained respectively for pre- and post-transmigration (Figure 37B).

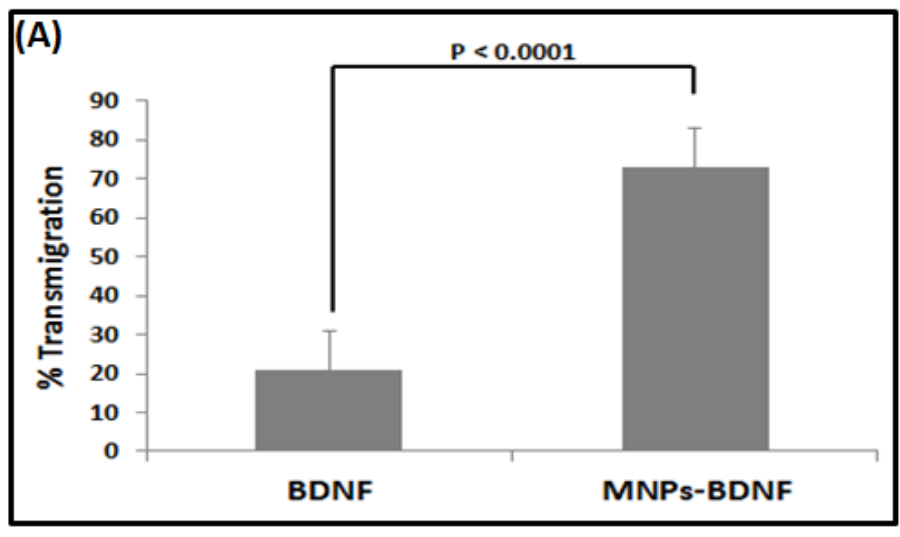

\begin{tabular}{|lll|}
\hline \hline B) & Before Treatment & After Treatment \\
\hline Untreated & $287.6 \pm 9.4$ & $286.67 \pm 7.2$ \\
MNP-BDNF treatment & $280.4 \pm 10.4$ & $276.56 \pm 9.7$ \\
\hline
\end{tabular}

Figure 37: (A). Percentage transmigration of free and MNP-BDNF nanoformulations. (B). TEER values of the in vitro BBB model before and after treatment of free and MNPBDNF nanoformulations. 
Furthermore, we studied whether transmigrated BDNF retains functional efficiency or not? As such, CREB expression in the astrocytes on the basal side of BBB was quantified. Consistent with the transmigration ability, we found that treatment with BDNF alone did not induce any change in CREB expression in astrocytes in the basal side. In contrast, treatment with MNP-BDNF showed an upregulation of CREB (TAI=1.67 $\pm 0.12 ; p=0.007$, figure 38$)$. This result confirmed that unlike free BDNF, BDNF bound to MNP is able to cross BBB and is effective in its function. Since astrocytes of established in vitro BBB model remains in direct contact of media from lower chamber, it is expected that transmigrated BDNF in the lower chamber will exert its effect on these cell. In the same line, magnetic drug targeting of anticancer drugs to treat brain carcinoma has already been reported (Chertok et al., 2008). Thus, targeted delivery of BDNF in brain through an effective carrier such as MNPs would provide significant therapeutic benefits for treatment drug addiction and/or neuroAIDS.

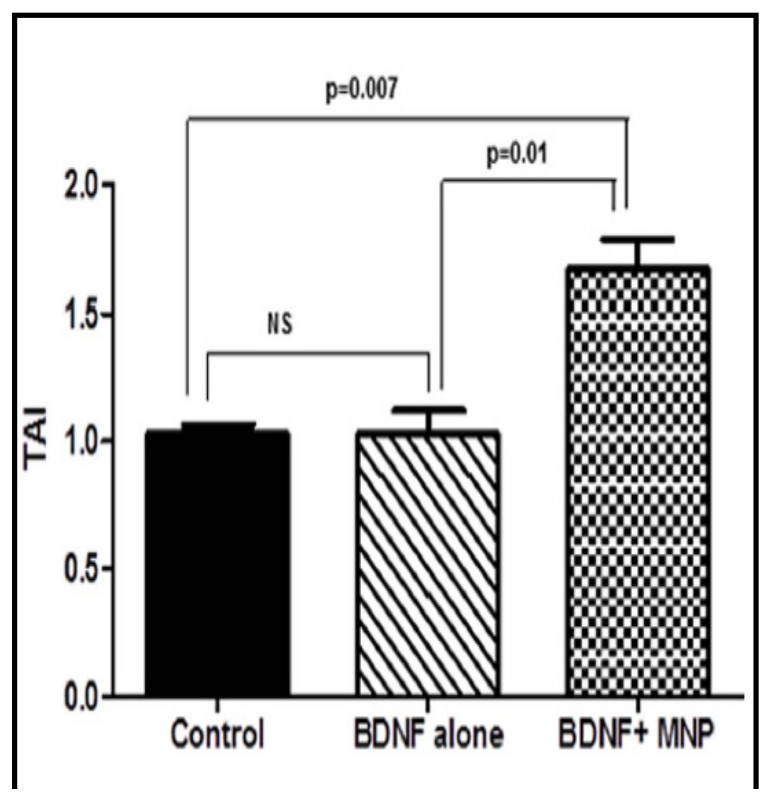

Figure 38: Trans-accumulation index (TAI) showing effect of Free BDNF and MNPs-BDNF nanoformulations on CREB expression in Astrocytes across BBB. CREB expression is significantly upregulated upon treatment of MNP-BDNF nanoformulation which suggest effective transmigration of BDNF across BBB via nanocarrier. 


\section{Summary:}

As stated by President Nixon in 1971, drug abuse is America's number one enemy. In fact, drug abuse is a serious global problem which speeds up the spread of many blood-borne diseases including HIV. Considering the highintensity of affected populations, lack of treatment, and unrepairable social costs associated with the drug abuse and HIV infection, it is quintessential to develop better strategies for their preventions. In this dissertation I investigated a novel MNPs-based drug delivery system for delivery of anti-addiction, and neuroprotective drugs across the BBB. It is anticipated that the active from of these drugs could be delivered across BBB. Successful delivery of anti-addiction agents e.g. CTOP (an opioid antagonist) may reduce the addictive effect of abusive drugs and block the synergistic neurotoxicity during HIV infection. Similarly, delivery of neuroprotective agent, brain derived neurotropic factor (BDNF), could be of therapeutic benefit in the treatment of both, drug addiction and HIV infection. Further, ultrasmall magnetic nanoparticles was synthesized and loaded into PEGylated liposome which could provide nanoplatform for enhanced BBB transmigration of drugs. Drugs bound to naked MNPs may either be decomposed due to metabolic activity of peripheral circulation (blood) or be cleared or entraped by reticuloendothelial systems (liver, stomach, gut, lymphoid, etc.) before it reach to the brain. In this contrext, magnetized-liposome (liposomal encapsulation of MNPs bound drugs) is advantageous in many ways: (a) Liposomes coating protects drugs from metabolic insults of blood circulation and reduces their uptake by liver, stomach, gut, lymphoid, etc. This remarkably 
improves the stability and bioavailability of drugs in body. (b) Magnetized liposomes can be easily transported across BBB under influence of external magnetic force. (c) While drugs attached with MNPs are encapsulated in the liposomal core, additional free drugs can also be supplemented on the liposomes wall (phospholipid bilayers). Thus, per unit drug-loading efficiency of magnetized nanocarrier is enhanced.

Our developed nanocarriers seem to deliver improved level of therapeutics in the brain which is the common target organ for both HIV and drug abuse. It is expected that using our innovations, suppression of pathogenesis in drug addicted and HIV patients could be significantly improved and, in turn, it may add to achieve near-normal life expectancy for treated individuals. Thus, transfer of this strategy in clinical settings may be beneficial for these major social problems. A proposed model of drug delivery via magnetic nanocarriers has been shown in figure 39. Importantly, this strategy could be applied to other brain diseases leading to a healthier lifestyle. Many brain diseases such as Huntington's, Epilepsy, Stroke, Parkinson's, Alzheimer's, Multiple Sclerosis, Brain Tumors, etc. remain untreated, mainly due to impenetrability of drug or existing drug-delivery techniques across BBB. Thus, our magnetic nanocarriers could have universal applicability for drug-targeting in the brain in a non-invasive manner for many brain diseases. Additionally, magnetic field generated by MNPs under the influence of magnetic force could stimulate inactivated neurons in Parkinson's, Alzheimer's Stroke, etc. MNPs could also be used for imaging via MRI which can tell about pathological conditions and its progression. Thus, 
MNPs could be developed as a polypharmacological technique where it could simultaneously deliver drugs, diagnose pathological progression, and stimulate degenerating neurons in various brain diseases.

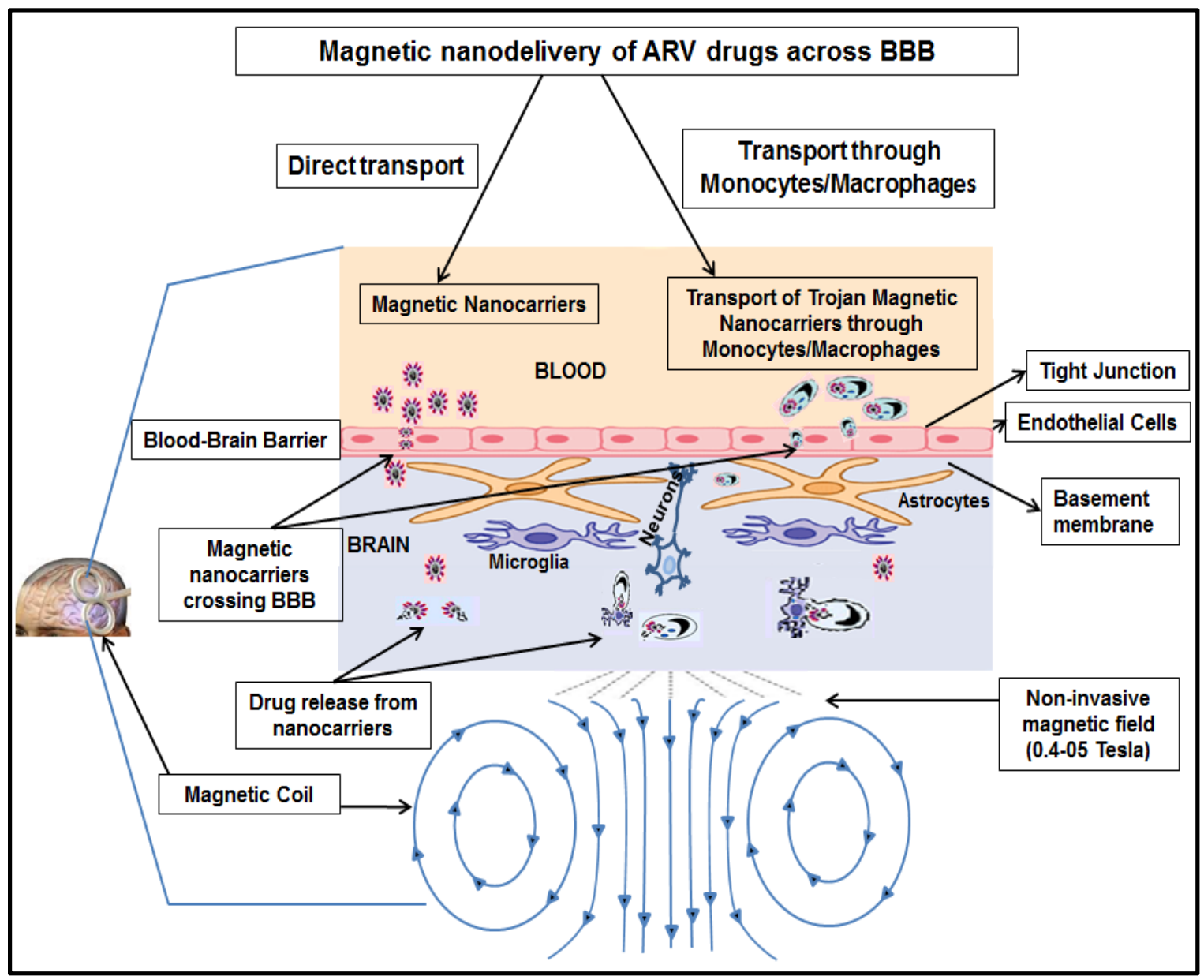

Figure 39: Proposed shcematic of magnetic nanoparticles based drugs delivery across BBB. 


\section{Future directions}

Based on the in vitro observations we assume that, under the influence of external magnetic force, drugs loaded magnetic nanocarriers in the form of magnetized-liposomes can either directly transport across the BBB. As such, magnetic hybrid nanoformulations (magnetized-liposomes) seem to be more practical nanovehicles for drug delivery to the brain and its applicability in animal model must take place. It will allow knowing the feasibility of our developed nanocarrier to go across the BBB in the physiological relevance condition. Following completion of work on mouse model, its applicability in monkey model must be evaluated; success of which may lead to the clinical applications of our developed nanocarriers (figure 40). It is expected that using our innovations, suppression of pathogenesis in drug addicted and HIV patients could be significantly improved and, in turn, it may add to achieve near-normal life expectancy for treated individuals. Thus, transfer of this strategy in clinical settings may be beneficial for these major social problems. Importantly, this strategy could be applied to other brain diseases leading to a healthier lifestyle.

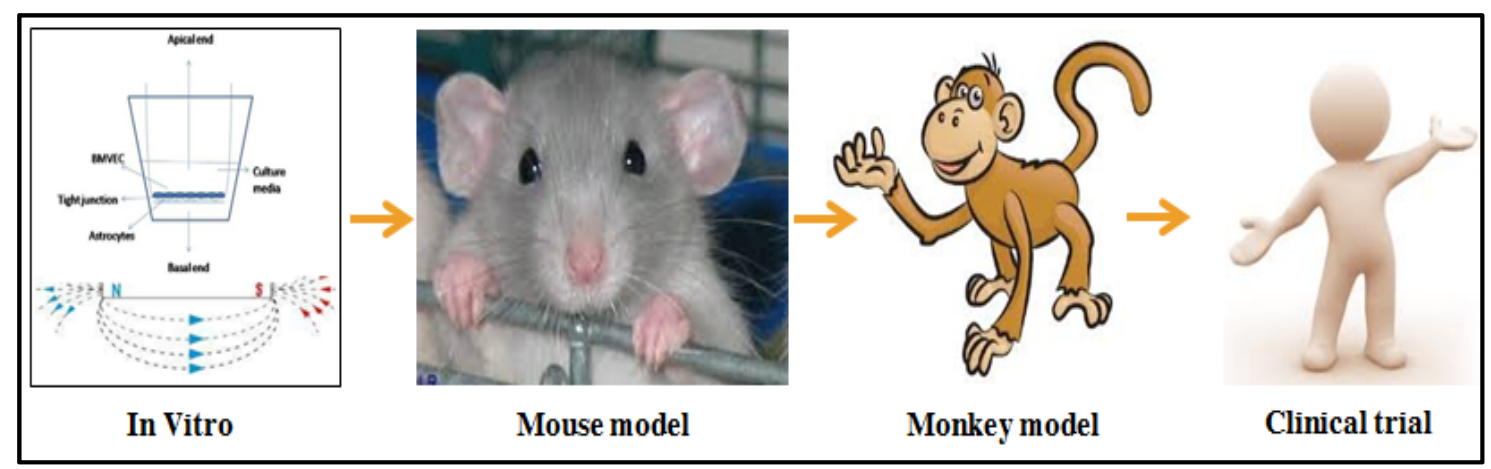

Figure 40. Schematic of future work with nanoformulations from in vitro study to the clinical trial (Google images ). 
7. References:

1. Abbott N.J., Rönnbäck L., Hansson E. (2006) Astrocyte-endothelial interactions at the blood-brain barrier. Nat. Rev. Neurosci. 7:41-53.

2. AIDSinfo-NIH (2012), HIV and Its Treatment - FDA-Approved Anti-HIV Medications:

http://aidsinfo.nih.gov/contentfiles/ApprovedMedstoTreatHIV_FS_en.pdf

3. Albright A.V., Shieh J.T., O'Connor M.J., Gonzalez-Scarano F. (2000) Characterization of cultured microglia that can be infected by HIV-1. J Neurovirol. 6:S53-S60.

4. Alexiou C., Schmid R.J., Jurgons R., Kremer M., Wanner G., Bergemann C., Huenges E., Nawroth T., Arnold W., Parak F.G. .(2006) Targeting cancer cells: magnetic nanoparticles as drug carriers. Eur Biophys.J 35: 446-450.

5. Al-Ghananeem A.M., Saeed H., Florence R., Yokel R.A., Malkawi A.H. (2010) Intranasal drug delivery of didanosine-loaded chitosan nanoparticles for brain targeting; an attractive route against infections caused by aids viruses. J Drug Target.18:381-388.

6. Allen T.M., Sapra P., Moase E., Moreira J., Iden D. (2002) Adventures in targeting. J Liposome Res.12:5-12.

7. Almeida S.M., Letendre S., Ellis R. (2006) Human immunodeficiency virus and the central nervous system. Braz J Infect Dis. 10:41-50.

8. Alonso M., Bekinschtein P., Cammarota M., Vianna M.R., Izquierdo I., Medina J.H. (2005) Endogenous BDNF is required for long-term memory formation in the rat parietal cortex. Learn Mem 12: 504-510.

9. Alyaudtin R.N., Reichel A., Löbenberg R., Ramge P., Kreuter J., Begley D.J. (2001) Interaction of poly(butylcyanoacrylate) nanoparticles with the bloodbrain barrier in vivo and in vitro. J. Drug Target. 9 209-221.

10. Amyere M., Mettlen M., Van Der Smissen P., Platek A., Payrastre B., Veithen A., Courtoy P.J. (2002) Origin, originality, functions, subversions and molecular signalling of macropinocytosis. Int J Med Microbiol 291: 487-494.

11. An, S.F. and Scaravilli, F (1997) Early HIV-1 infection of the central nervous system. Arch Anat Cytol Pathol , 45 (2-3) 94 - 105.

12. Antinori A., Arendt G., Becker J.T., Brew B.J., Byrd D.A., Cherner M., Clifford D.B., Cinque P., Epstein L.G., Goodkin K., Gisslen M., Grant I., 
Heaton R.K., Joseph J., Marder K., Marra C.M., McArthur J.C., Nunn M., Price R.W., Pulliam L., Robertson K.R., Sacktor N., Valcour V., Wojna V.E. (2007) Updated research nosology for HIV-associated neurocognitive disorders . Neurology. 69: $1789-1799$.

13. Arnaiz B., Martínez-Ávila O., Falcon-Perez J.M., Penadeś S. (2012) Cellular Uptake of Gold Nanoparticles Bearing HIV gp120 Oligomannosides. Bioconjugate Chem. 23 (4): pp 814-825

14. Athanasiou K.A., Agarwal A., Muffoletto A., Dzida F.J., Constantinides G., Clem M. (1995) Biomechanical properties of hip cartilage in experimental animal models. Clin Orthop Relat Res. 316:254-266.

15. Atluri V. S. R., Pilakka-Kanthikeel S., Reddy P. V. B., Yndart A., Nair. (2013). Human Synaptic Plasticity Gene Expression Profile and Dendritic Spine Density Changes in HIV-Infected Human CNS Cells: Role in HIVAssociated Neurocognitive Disorders (HAND). PLOS ONE (2013), Volume 8 (4): e61399.

16. Bae, Y. and Kataoka, K. (2009) Intelligent polymeric micelles from functional poly(ethylene glycol)-poly(amino acid) block copolymers Advanced Drug Delivery Reviews. 61:768-784.

17. Balaji N., Meera Sheriffa Begum K.M., Anantharaman N., Uddin M. S. (2009) Adsorption and desorption of L-Phenylalanine on nano-sized magnetic particles, J. Eng. Appl. Sci. 4(8): 39-44.

18. Batrakova E.V., Li S., Alakhov V.Y., Miller D.W., Kabanov A.V., (2003) Optimal structure requirements for pluronic block copolymers in modifying Pglycoprotein drug efflux transporter activity in bovine brain microvessel endothelial cells, J. Pharmacol. Exp. Ther. 304: 845-854.

19. Batrakova E.V., Li S., Miller D.W., Kabanov A.V. (1999) Pluronic P85 increases permeability of a broad spectrum of drugs in polarized BBMEC and Caco-2 cell monolayers, Pharm. Res. 16:1366-1372.

20. Batrakova E.V., Gendelman H.E., Kabanov AV. (2011) Cell-mediated drug delivery. Expert Opin Drug Deliv.8:415-433.

21. Batrakova E.V., Li S., Reynolds A.D., Mosley, R.L., Bronich T.K., Kabanov A.V., Gendelman H.E. (2007) A macrophage-nanozyme delivery system for Parkinson's disease. Bioconjug Chem.18(5):1498-506.

22. Bawarski, W.E., Chidlowsky, E., Bharali, D.J., and Mousa, S.A. (2008) Emerging nanopharmaceuticals, Nanomedicine: Nanotechnology, Biology and Medicine.4: 273-282 
23. Behr J-P. (1997) The Proton Sponge: a Trick to Enter Cells the Viruses Did Not Exploit CHIMIA. International Journal for Chemistry.51(1):34-6.

24. Beltran J. A., Pallur A., Chang S. L. (2006) HIV-1 gp120 up-regulation of the $\mathrm{mu}$ opioid receptor in TPA-differentiated HL-60 cells. International Immunopharmacology. 6(9):1459-1467.

25. Bernabeu R., Bevilaqua L., Ardenghi P., Bromberg E., Schmitz P., Bianchin M., Izquierdo I., Median J.H. (1997) Involvement of hippocampal CAMP/CAMP-dependent protein kinase signaling pathways in a late memory consolidation phase of aversively motivated learning in rats. Proc Natl Acad Sci 94: 7041-7046.

26. Bestman-Smith J., Gourde P., D'esormeaux A., Tremblay M. J., and Bergeron M. G. (2000) Sterically stabilized liposomes bearing anti-HLA-DR antibodies for targeting the primary cellular reservoirs of HIV-1. Biochimica et Biophysica Acta. 1468(1-2):161-174.

27. Betz A.L., Firth J.A., Goldstein G.W. (1980) Polarity of the blood-brain barrier: distribution of enzymes between the luminal and antiluminal membranes of brain capillary endothelial cells. Brain Res 192:17-28.

28. Blume G., Cevc G. (2000) Molecular mechanism of the lipid vesicle longevity in vivo. Biochim Biophys Acta.1146:157-68.

29. Boison D. (2009) Adenosine augmentation therapies (AATs) for epilepsy: prospect of cell and gene therapies. Epilepsy Res.85(2-3):131-41.

30. Bourtchuladze R., Frenguelli B., Blendy J., Cioffi D., Schutz G., Silva A.J (1994) Deficient long-term memory in mice with a targeted mutation of the cAMP-responsive element-binding protein. Cell 79: 59-68.

31. Boven LA, Middel J, Breij EC, Schotte D, Verhoef J, Soderland C, Nottet HS. (2000) Interactions between HIV-infected monocyte-derived macrophages and human brain microvascular endothelial cells result in increased expression of CC chemokines. J Neurovirol. 6:382-389.

32. Bowman, M. C., Ballard, T. E., Ackerson, C. J., Feldheim, D. L., Margolis, D. M. and Melander, C. (2008) Inhibition of HIV fusion with multivalent gold nanoparticles J. Am. Chem. Soc. 130, 6896-6897.

33. Bramham C.R., Messaoudi E. (2005) BDNF function in adult synaptic plasticity: the synaptic consolidation hypothesis. Prog Neurobiol 76: 99-125.

34. Briz, O., Macias, R.I.R., Vallejo, M., Silva, A., Serrano, M.A., Marin, J.J.G. (2003) Usefulness of liposomes loaded with cytos tatic bile acid derivatives to circum-vent chemotherapy res is tance of enterohepatic tumors. Mol. Pharmacol. 63: 742-750. 
35. Brown J.M., Yamamoto BK. (2003) Effects of amphetamines on mitochondrial function: role of free radicals and oxidative stress. Pharmacol Ther. 99:45-53.

36. Brownson E.A., Abbruscato T.J., Gillespie T.J., Hruby V.J., Davis T.P. (1994) Effect of peptidases at the blood brain barrier on the permeability of enkephalin. J Pharmacol Exp Ther 270:675-680.

37. Brynskikh A.M., Zhao Y., Mosley R.L., Li S., Boska M.D., Klyachko N.L., Kabanov A.V., Gendelman H.E., Batrakova E.V. (2010) Macrophage delivery of therapeutic nanozymes in a murine model of Parkinson's disease. Nanomedicine (Lond). 5(3):379-96.

38. Bummer P.M. (2004) Physical chemical considerations of lipid-based oral drug deliverysolid lipid nanoparticles, Crit. Rev. Ther. Drug Carrier Syst. 21 : $1-20$.

39. Burdo T.H., Katner S.N., Taffe M.A., Fox H.S. (2006) Neuroimmunity, drugs of abuse, and neuroAIDS. Neuroimmune Pharmacol.1, 41-49.

40. Butt, A.M., Jones, H.C., Abbott, N.J. (1990). Electrical-resistance across the blood-brain barrier in anesthetized rats - a developmental study. J. Physiol. 429, 47-62.

41. Cammarota M., Bevilaqua L.R., Ardenghi P., Paratcha G., Levi de Stein M., Izquierdo I, Medina JH. (2000) Learning-associated activation of nuclear MAPK, CREB and Elk-1, along with Fos production, in the rat hippocampus after a one-trial avoidance learning: abolition by NMDA receptor blockade. Brain Res Mol Brain Res 76: 36-46.

42. Carvalho F.C., Mainardes R.M., Gremião M.P.D. (2011) Exploring the Nanotechnology-Based Drug Delivery Systems for AIDS Treatment. In: Kasenga, FH, editor. Understanding HIVIAIDS management and care pandemic approaches in the 21st century. Intechweb.org; pp. 367-384.

43. Castro-Nallar E., Perez-Losada M., Burton G.F., Crandall K.A. (2012) The evolution of HIV: Inferences using phylogenetics. Molecular Phylogenetics and Evolution. 62: 777-792.

44. Chapleau C.A., Carlo M.E., Larimore J.L., Pozzo-Miller L. (2008) The actions of BDNF on dendritic spine density and morphology in organotypic slice cultures depend on the presence of serum in culture media. J Neurosci Methods 169: 182-190.

45. Chattopadhyay N., Zastre J., Wong H.L., Wu X.Y., Bendayan R. (2008) Solid lipid nanoparticles enhance the delivery of the HIV protease inhibitor, 
atazanavir, by a human brain endothelial cell line, Pharm. Res. 25 : 22622271.

46. Chaughule, R.S., Purushotham, S., Ramanujan, R.V. (2012) Magnetic Nanoparticles as Contrast Agents for Magnetic Resonance Imaging. Proceedings of the national academy of sciences, India Section A: Physical Sciences 82 (3): 25-268.

47. Chen, F.H., Gao Q., Ni J.Z. (2008) The grafting and release behavior of doxorubincin from $\mathrm{Fe}_{3} \mathrm{O}_{4} @ \mathrm{SiO}_{2}$ core-shell structure nanoparticles via an acid cleaving amide bond: the potential for magnetic targeting drug delivery. Nanotechnology 19(16): p. 165103.

48. Cheng H., Kastrup C.J., Ramanathan R., Siegwart D.J., Ma M., Bogatyrev S.R., Xu Q., Whitehead K.A., Langer R., Anderson D.G. (2010) Nanoparticulate cellular patches for cell-mediated tumoritropic delivery. ACS Nano. 23:4(2):625-31.

49. Chertok B., Moffat B.A., David A.E., Yu F., Bergemann C., Ross B.D., Yang V.C. (2008) Iron oxide nanoparticles as a drug delivery vehicle for MRI monitored magnetic targeting of brain tumors. Biomaterials. 29(4):487-496.

50. Chomoucka J., Drbohlavova J., Huska D., Adam V., Kizek R., Hubalek J. (2010) Magnetic nanoparticles and targeted drug delivering, Pharmacological Research 62: 144-149.

51. Chun T., Justement J., Moir S., Hallahan C., Maenza J., Mullins J., Collier A., Corey L., Fauci A. (2007) Decay of the HIV reservoir in patients receiving antiretroviral therapy for extended periods: implications for eradication of virus. Journal of Infectious Diseases. 195: 1762-1764.

52. Chung, J.E., Yokoyama, M., Aoyagi, T., Sakurai, Y. and Okano, T. (1998) Effect of molecular architecture of hydrophobically modified poly ( $\mathrm{N}$ isopropylacrylamide) on the formation of thermoresponsive core-shell micellar drug carriers. J. Control. Release. 53, 119-130.

53. Citi S., Sabanay H., Jakes R., Geiger B., Kendrick-Jones J. (1988) Cingulin, a new peripheral component of tight junctions. Nature 333:272-276.

54. Conant K., McArthur J.C., Griffin D.E., Sjulson L., Wahl L.M., Irani D.N. (1999) Cerebrospinal fluid levels of MMP-2, 7, and 9 are elevated in association with human immunodeficiency virus dementia.Ann Neurol. 46:391-398.

55. Crone C., Christensen 0. (1981). Electrical resistance of a capillary endothelium. Journal of General Physiology. 77: 349-371. 
56. Cubells J.F., Rayport S., Rajendran G., Sulzer D. (1994) Methamphetamine neurotoxicity involves vacuolation of endocytic organelles and dopaminedependent intracellular oxidative stress. J Neurosci. 14:2260-2271.

57. D'Souza, S.S., DeLuca, P.P. (2006) Methods to assess in vitro drug release from injectable polymeric particulate systems. Pharm. Res. 23:460Y474.

58. Davis L.E., Hjelle B.L., Miller V.E., Palmer D.L., Llewellyn A.L., Merlin T.L., Young S.A., Mills R.G., Wachsman W., Wiley C.A. (1992) Early viral brain invasion in iatrogenic human immunodeficiency virus infection. Neurology 42:1736-39.

59. Davson H., Segal M.B. (1996). The return of the cerebrospinal fluid to the blood: The drainage mechanism. In: Anonymous Physiology of the CSF and Blood-Brain Barriers. CRC Press: Boca Raton, pp 489 -523.

60. Davson, H., Kleeman, C. R., Levin, E. (1961) Blood-brain barrier and extracellular space. J. Physiol. (Lond.)159.

61. Dechy-Cabaret O., Martin-Vaca B., Bourissou D. (2004) Controlled ringopening polymerization of lactide and glycolide, Chem. Rev. 104: 6147-6176.

62. Dehouck B., Dehouck M.P., Fruchart J.C., Cecchelli R. (1994) Upregulation of the low density lipoprotein receptor at the blood-brain barrier: intercommunications between brain capillary endothelial cells and astrocytes. J Cell Biol 126:465-473.

63. Deng L., KE X., He Z., Yang D., Gong H., Zhang Y., Jing X., Yao J., Chen J.A. (2012) MSLN-targeted multifunctional nanoimmunoliposome for MRI and targeting therapy in pancreatic cancer. International Journal of Nanomedicine. 7:5053-5065.

64. Denker B.M., Nigam S.K. (1998) Molecular structure and assembly of the tight junction. Am J Physiol 274:F1-9.

65. Desai, M.P., Labhasetwar, V., Amidon, G.L., Levy, R.J. (1996) Gastrointestinal Uptake of Biodegradable Microparticles: Effect of Particle Size Pharmaceutical Research, 13, 1838-1845.

66. Desai, M.P., Labhasetwar, V., Walter, E., Levy, R.J., Amidon, G.L. (1997) The Mechanism of Uptake of Biodegradable Microparticles in Caco-2 Cells Is Size Dependent Pharmaceutical Research, 14, 1568-1573.

67. Descamps L., Dehouck M.P., Torpier G., Cecchelli R. (1996) Receptormediated transcytosis of transferrin through blood-brain barrier endothelial cells. Am J Physiol 270:H1149-1158. 
68. Desormeaux A., Bergeron M.G. (1998) Liposomes as drug delivery system: a strategic approach for the treatment of HIV infection, J. Drug Target. 6:115.

69. Destache C.J., Belgum T., Goede M., Shibata A., Belshan M.A. (2010) Antiretroviral release from poly(DL-lactide-co-glycolide) nanoparticles in mice. J Antimicrob Chemother. 65(10):2183-2187.

70. Ding H., Yong K-T, Roy I., Pudavar H.E., Law W.C., Bergey E.J., Prasad P.N. (2007) Gold nanorods coated with multilayer polyelectrolyte as contrast agents for multimodal imaging. The Journal of Physical Chemistry C. 111(34): p. $12552-12557$.

71. Dou H., Destache C.J., Morehead J.R., Mosley R.L., Boska M.D., Kingsley J., Gorantla S., Poluektova L., Nelson J.A., Chaubal M., Werling J., Kipp J., Rabinow B.E., Gendelman H.E. (2006) Development of a macrophage-based nanoparticle platform for antiretroviral drug delivery. Blood. 5;108(8):2827-35.

72. Dou H., Grotepas C.B., McMillan J.M., Destache C.J., Chaubal M., Werling J., Kipp J., Rabinow B., Gendelman H.E. (2009). Macrophage delivery of nanoformulated antiretroviral drug to the brain in a murine model of neuroAIDS. J Immunol. 2009 Jul 1;183(1):661-9.

73. Douce V. Le, Janossy A., Hallay H., Ali S., Riclet R., Rohr O., Schwartz C. (2012) Achieving a cure for HIV infection: do we have reasons to be optimistic? Journal of Antimicrobial Chemotherapy. 67 (5): 1063-1074.

74. Dutta T., Jain N.K. (2007) Targeting potential and anti-HIV activity of lamivudine loaded mannosylated poly(propyleneimine) dendrimers. Biochim. Biophys. Acta. 1770:681-686.

75. Dzmitruk V., Shcharbin D., Pedziwiatr E., Bryszewska M. (2011). Dendrimers in Anti-HIV Therapy, Advances in Nanocomposite Technology, Abbass Hashim (Ed.), ISBN: 978-953-307-347-7.

76. Eisenstein T.K., Hilburger M.E. (1998) Opioid modulation of immune responses: effects on phagocyte and lymphoid cell populations," Journal of Neuroimmunology, vol. 83, no. 1-2, pp. 36-44.

77. Elechiguerra J.L., Burt J.L., Morones J.R., Camacho-Bragado A., Gao X., Lara H.H., Yacaman M.J. (2005) Interaction of AgNPs with HIV-1.J Nanobiotechnology. 3:6.

78. El-Hage N., Gurwell J. A., Singh I. N., Knapp P. E., Nath A., Hauser K. F. (2005) Synergistic increases in intracellular $\mathrm{Ca2}+$, and the release of MCP-1, RANTES, and IL- 6 by astrocytes treated with opiates and HIV-1 Tat. Glia 50 , 91-106. 
79. Enting R.H., Hoetelmans R.M.W., Lange J.M.A., Burger D.M., Beijnen J.H., Portegies P. (1998) Antiretroviral drugs and the central nervous system. AIDS.12:1941-1955.

80. Escribano, E., Fernández-Pacheco, R., Valdivia, J. G., Ibarra, M. R., Marquina, C., Queralt, J. (2012) Effect of magnet implant on iron biodistribution of Fe@C nanoparticles in the mouse. Arch. Pharm. Res., 35, 93-100.

81. Ferris M.J, Mactutus C.F, Booze R.M. (2008) Neurotoxic profiles of HIV, psychostimulant drugs of abuse, and their concerted effect on the brain: current status of dopamine system vulnerability in NeuroAIDS. Neurosci Biobehav Rev.32:883-909.

82. Fischer-Smith T., Rappaport J. (2005) Evolving paradigms in the pathogenesis of HIV-1-associated dementia. Expert Rev Mol Med. 7:1-26.

83. Fishman J.B., Rubin J.B., Handrahan J.V., Connor J.R., Fine RE (1987) Receptor-mediated transcytosis of transferrin across the blood-brain barrier. $\mathrm{J}$ Neurosci Res 18:299-304.

84. Frankfurt M., Salas-Ramirez K., Friedman E., Luine V. (2011) Cocaine alters dendritic spine density in cortical and subcortical brain regions of the postpartum and virgin female rat. Synapse 65: 955-961.

85. Frascione, D., Diwoky C., Almer G., Opriessnig P., Vonach C., Gradauer K., Leitinger G., Mangge H., Stollberger R., Prassl R. (2012) Ultrasmall superparamagnetic iron oxide (USPIO)-based liposomes as magnetic resonance imaging probes. International Journal of Nanomedicine. 7(1): p. 2349-2359.

86. Freiberg S., Zhu X.X. (2004) Polymer microspheres for controlled drug release International Journal of Pharmaceutics. 282: 1-18.

87. Freier D.O., Fuchs B.A. (1993) Morphine-induced alterations in thymocyte subpopulations of B6C3F1 mice. J Pharmacol Exp Ther 265: 81-88.

88. Fuchs B.A., Pruett S.B. (1993) Morphine induces apoptosis in murine thymocytes in vivo but not in vitro: involvement of both opiate and glucocorticoid receptors. J Pharmacol Exp Ther 266: 417-423.

89. Fujimura, R.K., Bockstahler, L.E., Goodkin, K., Werner, T., BrackWerner, R., Shapshak, P. (1996). Neuropathology and virology of HIV associated dementia. Rev. Med. Virol. 6, 141-150. 
90. Furuse M., Sasaki H., Tsukita S. (1999) Manner of interaction of heterogeneous claudin species within and between tight junction strands. J Cell Biol 147:891-903.

91. Galanzha E.I., Shashkov E.V., Kelly T., Kim J.W., Yang L., Zharov V.P. (2009) In vivo magnetic enrichment and multiplex photoacoustic detection of circulating tumor cells. Nature Nanotechnol 12:855-860.

92. Gandhi N., Saiyed Z., Thangavel S., Rodriguez J., Rao K.V., Nair M.P. (2009). Differential effects of HIV type 1 clade Band clade C Tat protein on expression of proinflammatory and antiinflammatory cytokines by primary monocytes. AIDS Res Hum Retroviruses 25: 691-699.

93. Gandhi N., Saiyed Z.M., Napuri J., Samikkannu T., Reddy P.V., Agudelo M, Khatavkar P, Saxena SK, Nair MP. (2010) Interactive role of human immunodeficiency virus type 1 (HIV-1) clade-specific Tat protein and cocaine in blood-brain barrier dysfunction: implications for HIV-1-associated neurocognitive disorder. J Neurovirol 16: 294-305.

94. Gao X., Tao W., Lu W., Zhang Q., Zhang Y., Jiang X., Fu S. (2006) Lectinconjugated PEG-PLA nanoparticles: preparation and brain delivery after intranasal administration, Biomaterials 27: 3482-3490.

95. Gao X., Wu B., Zhang Q., Chen J., Zhu J., Zhang W., Rong Z., Chen H., Jiang X. (2007) Brain delivery of vasoactive intestinal peptide enhanced with the nanoparticles conjugated with wheat germ agglutinin following intranasal administration, J. Control. Release. 121:156-167.

96. Garcia P, Youssef I., Utvik J.K, et al. (2010) Ciliary neurotrophic factor cellbased delivery prevents synaptic impairment and improves memory in mouse models of Alzheimer's disease. J Neurosci. 2:30(22):7516-27.

97. Gaucher, G., Dufresne, M.H., Sant, V.P., Kang, N., Maysinger, D., and Leroux, J.C. (2005) Block copolymer micelles: preparation, characterization and application in drug delivery Journal of Controlled Release. 109: 169-188.

98. Ghafouri M., Amini S., Khalili K., Sawaya B.E. (2006) HIV-1 associated dementia: symptoms and causes.Retrovirology. 3:28.

99. Giri N., Shaik N., Pan G., Terasaki T., Mukai C., Kitagaki S., Miyakoshi N., Elmquist W.F. (2008) Investigation of the role of breast cancer resistance protein (Bcrp/Abcg2) on pharmacokinetics and central nervous system penetration of abacavir and zidovudine in the mouse. Drug Metab. Dispos. $36: 1476-1484$.

100.Gittins D.I., Caruso F. (2008) Tailoring the polyelectrolyte coating of metal nanoparticles. The Journal of Physical Chemistry B, 2001. 105(29): p. 68466852. 
101.Gonatas N.K., Stieber A., Hickey W.F., Herbert S.H., Gonatas J.O. (1984) Endosomes and Golgi vesicles in adsorptive and fluid phase endocytosis. J Cell Biol. 99:1379-1390.

102.Gonzalez-Mariscal L., Betanzos A., Nava P., Jaramillo B.E. (2003) Tight junction proteins. Prog Biophys Mol Biol. 81: 1-44.

103.(Google images $_{\text {) }}$, https://www.google.com/imghp?hl=en\&tab=ii\&authuser=0

104.Goswami R, Dawson SA, Dawson G (1998) Cyclic AMP protects against staurosporine and wortmannin-induced apoptosis and opioid-enhanced apoptosis in both embryonic and immortalized (F-11kappa7) neurons. J Neurochem 70: 1376-1382.

105.Gray F, Scaravilli F, Everall I, Chretien F, An S,et al. 1996. Neuropathology of early HIV-1infection. Brain Pathol. 6:1-15.

106.Griffiths PD. A perspective on antiviral resistance. J Clin Virol. 2009;46:3-8.

107.Gunaseelan S, Gunaseelan K, Deshmukh M, Zhang X, Sinko PJ. Surface modifications of nanocarriers for effective intracellular delivery of anti-HIV drugs. Adv Drug Deliv Rev. 2010;62(4-5):518-531.

108.Gupta A., Zhang Y., Unadkat J.D., Mao Q. HIV protease inhibitors are inhibitors but not substrates of the human breast cancer resistance protein (BCRP/ABCG2) J. Pharmacol. Exp. Ther. 2004;310:334-341.

109.Gupta, U., Jain, N.K., 2010. Non-polymeric nano-carriers in HIVIAIDS drug delivery and targeting. Non-polymeric nano-carriers in HIVIAIDS drug delivery and targeting. Adv. Drug Deliv. Rev. 62, 478-490.

110.Hamilton R.D., Foss A.J., Leach L., (2007) ;Establishment of a human in vitro model of the outer blood-retinal barrier, J. Anat. 211- 707.

111.Haorah J., Heilman D., Diekmann C., Osna N., Donohue T. M. Jr, Ghorpade A. and Persidsky Y. (2004) Alcohol and HIV decrease proteasome and immunoproteasome function in macrophages: implications for impaired immune function during disease. Cell Immunol. 229, 139-148.

112. Haorah J., Knipe B., Leibhart J., Ghorpade A. and Persidsky Y. (2005) Alcohol-induced oxidative stress in brain endothelial cells causes blood-brain barrier dysfunction. J. Leukoc. Biol. 78, 1223-1232.

113. Haskins J, Gu L, Wittchen ES, Hibbard J and Stevenson BR (1998) ZO-3, a novel member of the MAGUK protein family found at the tight junction, interacts with ZO-1 and occludin. J Cell Biol 141:199-208. 
114. Hau V S, "Effect of peripheral inflammatory pain on the blood-brain barrier" the University of Arizona Electronic Theses and Dissertations.

115.Hauser K. F., El-Hage N., Buch S., Berger J. R., Tyor W. R., Nath A., BruceKeller A. J. and Knapp P. E. (2005) Molecular targets of opiate drug abuse in neuroAIDS. Neurotox. Res. 8, 63-80.

116. Hauser KF, El-Hage N, Stiene-Martin A, Maragos WF, Nath A, et al. (2007) HIV-1 neuropathogenesis: glial mechanisms revealed through substance abuse. J Neurochem 100: 567-587.

117. Hawkins, B. T. and T.P. Davis. The blood-brain barrier/neurovascular unit in health and disease. Pharmacological Reviews 57(2):173-185, 2005

118. Hendriks JJ, Teunissen CE, de Vries HE, et al. Macrophages and neurodegeneration. Brain Res Brain Res Rev. 2005 Apr;48(2):185-95.

119. Hirase T, Staddon JM, Saitou M, Ando-Akatsuka Y, Itoh M, Furuse M, Fujimoto K, Tsukita S and Rubin LL (1997) Occludin as a possible determinant of tight junction permeability in endothelial cells. J Cell Sci 110 (Pt 14):1603-1613.

120.Ho DD, Rota TR, Schooley R, Kaplan JC, Allan JD, Groopman JE, Resnick L, Felsenstein D, Andrews CA, Hirsch M (1985), Isolation of HTLV-III from cerebrospinal fluid and neural tissues of patients with neurologic syndromes related to the acquired immunodeficiency syndrome. N Engl J Med 313: 1493-1497.

121. Hofmann A, Wenzel D, Becher UM, Freitag DF, Klein AM, Eberbeck D, Schulte M, Zimmermann K, Bergemann C, Gleich B, Roell W, Weyh T, Trahms L, Nickenig G, Fleischmann BK, Pfeifer A (2009) Combined targeting of lentiviral vectors and positioning of transduced cells by magnetic nanoparticles. Proc Natl Acad Sci USA 106: 44-49.

122. Holt JL, Kraft-Terry SD, Chang L (2012) Neuroimaging studies of the aging of HIV-1-infected brain. J Neurovirol, 18 (4):291-302.

123.Ikehara $\mathrm{Y}$, Niwa $\mathrm{T}$, Biao $\mathrm{L}$, et al. A carbohydrate recognition-based drug delivery and controlled release system using intraperitoneal macrophages as a cellular vehicle. Cancer Res. 2006 Sep 1;66(17):8740-8.

124.ILO (2012), http://www.unaids.org/en/aboutunaids/unaidscosponsors/ilo/.

125.Izumikawa, S., Yoshioka, S., Aso, Y., and Takeda, Y. 1991, Preparation of poly(I-lactide) microspheres of different crystalline morphology and effect of 
crystalline morphology on drug release rate Journal of Controlled Release, $15,133-140$.

126.Jain S, Mishra V, Singh P, Dubey PK, Saraf DK, Vyas SP. RGD-anchored magnetic liposomes for monocytes/neutrophils-mediated brain targeting. Int $\mathrm{J}$ Pharm. 2003;261:43-55.

127.Jain T.K., Reddy M.K., Morales M.A., Leslie-Pelecky D.L., Labhasetwar V., Biodistribution, clearance, and biocompatibility of iron oxide magnetic nanoparticles in rats. Mol Pharmacol, 5 (2008), pp. 316-327.

128.Jain, K.K. 2008, Drug Delivery Systems: An Overview 437, 1-50

129.Jayapaul, J., et al., FMN-coated fluorescent iron oxide nanoparticles for RCP-mediated targeting and labeling of metabolically active cancer and endothelial cells. Biomaterials, 2011. 32(25): p. 5863-5871.

130.Jiménez, J.L., Clemente, M.I., Weber, N.D., Sanchez, J., Ortega, P., de la Mata, F.J., Gómez, R., García, D., López-Fernández, L.A., MuñozFernández, M.A. (2010). Carbosilane dendrimers to transfect human astrocytes with small interfering RNA targeting human immunodeficiency virus. BioDrugs, Vol. 24, pp. 331-343.

131.Jin S.X., Bi D.Z., Wang J., Wang Y.Z., Hu H.G., Deng Y.H., Pharmacokinetics and tissue distribution of zidovudine in rats following intravenous administration of zidovudine myristate loaded liposomes, Pharmazie 60 (2005) 840-843.

132.Johanson CE, Stopa E, McMillan PN (2011) The blood-cerebrospinal fluid barrier: structure and functional significance. In: Nag $S$ (ed) The blood-brain and other neural barriers, vol 686. Springer, New York, p 101-131.

133.Jones, M.C. and Leroux, J.C. 1999, Polymeric micelles - a new generation of colloidal drug carriers European Journal of Pharmaceutics and Biopharmaceutics, 48, 101-111.

134.Jun, Y.-w., et al., Nanoscale Size Effect of Magnetic Nanocrystals and Their Utilization for Cancer Diagnosis via Magnetic Resonance Imaging. Journal of the American Chemical Society, 2005. 127(16): p. 5732-5733.

135.Jung N., Lehmann C., Rubbert A., Knispel M., Hartmann P., van Lunzen J., Stellbrink H.J., Faetkenheuer G., Taubert D. Relevance of the organic cation transporters 1 and 2 for antiretroviral therapy in HIV infection. Drug Metab. Dispos. 2008;36:1616-1623. 
136.Kabanov A.V., Alakhov V.Y., Pluronic block copolymers in drug delivery: from micellar nanocontainers to biological response modifiers, Crit. Rev. Ther. Drug Carrier Syst. 19 (2002) 1-72.

137.Kanmogne GD, Singh S, Roy U, Liu X, McMillan J, Gorantla S, Balkundi S, Smith N, Alnouti Y, Gautam N, Zhou Y, Poluektova L, Kabanov AV, Bronich T, Gendelman HE (2012) Mononuclear phagocyte intercellular crosstalk facilitates transmission of celltargeted nanoformulated antiretroviral drugs to human brain endothelial cells. International Journal of Nanomedicine, 2012; 7: 2373-2388.

138.Kaplan IM, Wadia JS, and Dowdy SF (2005) Cationic TAT peptide transduction domain enters cells by macropinocytosis. $J$ Control Release 102: 247-253.

139.Katragadda A, Bridgman R, Betageri G. Effect of liposome composition and cholesterol on the cellular uptake of stavudine by human monocyte/macrophages. Cell Mol Biol Lett. 2000;5:483-494.

140.Kaul M, Garden GA, Lipton SA (2001) Pathways to neuronal injury and apoptosis in HIV-associated dementia. Nature 410: 988-994.

141.Khalil IA, Kogure K, Akita H, Harashima H. Uptake pathways and subsequent intracellular trafficking in nonviral gene delivery. Pharmacol Rev 2006 Mar; 58(1):32-45.

142.Kim D.H. and Martin D.C., Sustained release of dexamethasone from hlydrophilic matrices using PLGA nanoparticles for neural drug delivery, Biomaterials 27 (2006) 3031-3037.

143.Kim H.R., Andrieux K., Gil S., Taverna M., Chacun H., Desmaële D., Taran F., Georgin D., Couvreu P., Translocation of poly(ethylene glycol-cohexadecyl) cyanoacrylate nanoparticles into rat brain endothelial cells: role of apolipoproteins in receptor-mediated endocytosis, Biomacromolecules 8 (2007) 793-799.

144.Kim R.B., Fromm M.F., Wandel C., Leake B., Wood A.J., Roden D.M., Wilkinson G.R. The drug transporter P-glycoprotein limits oral absorption and brain entry of HIV-1 protease inhibitors. J. Clin. Invest. 1998;101:289-294.

145.Kim S., Scheerer S., Geyer M.A., Howell S.B., Direct cerebrospinal fluid delivery of an antiretroviral agent using multivesicular liposomes, J. Infect. Dis. 162 (1990) 750-752.

146.King GL and Johnson SM (1985) Receptor-mediated transport of insulin across endothelial cells. Science 227:1583-1586. 
147.Kohori, F., Sakai, K., Aoyagi, T., Yokoyama, M., Sakurai, Y. and Okano, T. (1998) Preparation a characterization of thermally responsive block copolymer micelles compris-ing poly ( $\mathrm{N}$-isopropylacrylamide- $\beta$-DL-lactide). J. Con-trol. Release, 55, 87-98.

148.Kondo, N., Iwao, T., Kikuchi, K.M., Shu, H., Yamanouchi, K., Yokoyama, K., Ohyama, K., and Ogyu, S. 1993, Pharmacokinetics of micronized, poorly water-soluble drug, HO-221, in exparimental animals Biological and pharmaceutical bulletin, 16, 796-800.

149.Koning, G. A.; Krijger, G. C. Targeted multifunctional lipid-based nanocarriers for image-guided drug delivery Anticancer Agents Med. Chem. 20077425440.

150.Koukourakis M.I., Koukouraki S., Giatromanolaki A., Kakolyris S., Georgoulias V., Velidaki A., Archimandritis S., Karkavitsas N.N., High intratumoral accumulation of stealth liposomal doxorubicinin sarcomasrationale for combination with radiotherapy, Acta Oncol. 39 (2000) 207-211.

151.Kozal MJ. Drug-resistant human immunodefiency virus. Clin Microbiol Infect. 2009;15(Suppl 1):69-73.

152.Koziara, J.M., Lockman, P.R., Allen, D.D., and Mumper, R.J. 2003, In Situ Blood-Brain Barrier Transport of Nanoparticles Pharmaceutical Research, 20, 1772-1778.

153.Kraft-Terry SD, Stothert AR, Buch S, Gendelman HE. (2010) neuroimmunity in the era of antiretroviral therapy. Neurobiol Dis, 37:542-8.

154.Kramer-Hammerle S, Rothenaigner I, Wolff H, Bell JE, Brack-Werner R. (2005), Cells of the central nervous system as targets and reservoirs of the human immunodeficiency virus. Virus Res.; 111:194-213.

155.Krantz A. Red cell-mediated therapy: opportunities and challenges. Blood Cells Mol Dis.1997;23(1):58-68.

156.Kreuter J. Nanoparticles as drug delivery systems. Encyclopedia of nanoscience and nanotechnology. 2004;7:161-180.

157.Kuo Y.C. and Kuo C.Y., Electromagnetic interference in the permeability of saquinavir across the blood-brain barrier using nanoparticulate carriers, Int. J. Pharm. 351 (2008) 271-281.

158.Kuo Y.C. and Su F.L., Transport of stavudine, delavirdine, and saquinavir across the blood-brain barrier by polybutylcyanoacrylate methyl methacrylate sulfopropyl methacrylate and solid lipid nanoparticles, Int. J. Pharm. 340 (2007) 143-152. 
159.Kuo Y.C. Chen H.H. (2006). Effect of nanoparticulate polybutylcyanoacrylate and methylmethacrylate-sulfopropylmethacrylate on the permeability of zidovudine and lamivudine across the in vitro blood-brain barrier. International Journal of Pharmaceutics, Vol. 327, pp. 160-69.

160.Kuo Y.C. Su FL. (2007). Transport of stavudine, delavirdine, and saquinavir across the blood-brain barrier by polybutylcyanoacrylate, methylmethacrylatesulfopropylmethacrylate, and solid lipid nanoparticles. International Journal of Pharmaceutics, Vol. 340, pp. 143-152.

161.Kwon M, Fernandez JR, Zegarek GF, Lo SB, Firestein BL BDNF-promoted increases in proximal dendrites occur via CREB-dependent transcriptional regulation of cypin. (2011) J Neurosci 31: 9735-9745.

162.Kyrtatos PG, Lehtolainen P, Junemann-Ramirez M, Garcia-Prieto A, Price AN, Martin JF, Gadian DG, Pankhurst QA, Lythgoe MF. Magnetic tagging increases delivery of circulating progenitors in vascular injury. JACC Cardiovasc Interv. 2009; 2: 794-802.

163.Costantino L., Tosi G., Ruozi B., Bondioli L., Vandelli M.A., Forni F., Colloidal systems for CNS drug delivery, in: H.S. Sharma (Ed.), Prog. Brain Res., 180, 2009, pp. 35-69.

164.Lanao J.M., Briones E., Colino C.I., Recent advances in delivery systems for antiHIV1 therapy, J. Drug Target. 15 (2007) 21-36.

165.Langer, R. 1976, Polymers for the sustained release of proteins and other macromolecules Nature, 263, 797-800.

166.Lara H H, Ixtepan-Turrent L, Treviño E N G, Singh D K. (2011), Use of silver nanoparticles increased inhibition of cell-associated HIV-1 infection by neutralizing antibodies developed against HIV-1 envelope proteins. J Nanobiotechnology. 2011; 9: 38.

167.Lara HH, Ayala-Nunez NV, Ixtepan-Turrent L, Rodriguez-Padilla C. Mode of antiviral action of AgNPs against HIV-1. J Nanobiotechnology. 2010;8:1.

168.Lefèvre CT, Menguy N, Abreu F, Lins U, Pósfai M, et al. (2011) A Cultured Greigite-Producing Magnetotactic Bacterium in a Novel Group of SulfateReducing Bacteria. Science 334: 1720-1723.

169.Letendre SL, Ellis RJ, Everall I, Ances, B, M., Bhatri, A., McCutchan, J.A. (2009), Neurologic complications of HIV disease and their treatment. Top HIV Med; 17: 46-56. 
170.Li Y, Wang H, Niu L, Zhou Y (2007) Chronic morphine exposure alters the dendritic morphology of pyramidal neurons in visual cortex of rats. Neurosci Lett 418: 227-231.

171.Liu Q, Shen Y, Chen J, Gao X, Feng C, Wang L, Zhang Q and Jiang X Nose-to-Brain Transport Pathways of Wheat Germ Agglutinin Conjugated PEG-PLA Nanoparticles. Pharm Res (2012) 29:546-558.

172.Lopez J. A., González F., Bonilla F.A., Zambrano G., Gómez M. E. (2010) Synthesis and characterization of $\mathrm{Fe}_{3} \mathrm{O}_{4}$ magnetic nanofluid. Revista Latinoamericana de Metalurgia y Materiales 30 (1): 60-66

173.Lossinsky AS, Shivers RR. Structural pathways for macromolecular and cellular transport across the blood-brain barrier during inflammatory conditions. Review. Histol Histopathol. 2004 Apr;19(2):535-64.

174.Lubbe AS, Bergemann C, Huhnt W, Fricke T, Riess H, et al. (1996) Preclinical experiences with magnetic drug targeting: tolerance and efficacy. Cancer Res 56: 4694-4701.

175.Luo, Jie - Phan, Trongha X - Yang, Yimei - Garelick, Michael G - Storm, Daniel R (2013). Increases in CAMP, MAPK activity, and CREB phosphorylation during REM sleep: implications for REM sleep and memory consolidation. The Journal of Neuroscience, 33 (15): 6460-6468.

176.Immordino M. L., Dosio F., and Cattel L. (2006) Stealth lipsomes: review of the basic science, rationale, and clinical applications, existing and potential. Int. J. Nanomed. 1:297-315.

177.Voinea M., E. Dragomir, I. Manduteanu, M. Simionescu, Binding and uptake of transferrin-bound liposomes targeted to transferrin receptors of endothelial cells, Vascul. Pharmacol. 39 (2002) 13-20.

178.Amiji M.M., T.K. Vyas, L.K. Shah, Role of nanotechnology in HIV/AIDS treatment: potential to overcome the viral reservoir challenge, Discov. Med. 6 (2006) 157-162.

179.Maeda, H., Wu, J., Sawa, Y., Matsumura, Y. and Hori, K. (2000) Tumor vascular permeability and the EPR effect in macromolecular therapeutics:a review. J. Control. Re-lease, 65, 271-284.

180.Mahajan S. D., Schwartz S. A., Shanahan T. C., Chawda R. P., and Nair M. P., "Morphine regulates gene expression of alpha- and beta-chemokines and their receptors on astroGlial cells via the opioid mu receptor," The Journal of Immunology, vol. 169, pp. 3589-3599, 2002. 
181.Mahajan SD, Schwartz SA, Aalinkeel R, Chawda RP, Sykes DE, et al. (2005) Morphine modulates chemokine gene regulation in normal human astrocytes. Clin Immunol 115: 323-332.

182.Mahato, R.I. 2007, Pharmaceutical dosage forms and drug delivery -300 .

183.Makadia, Hirenkumar K.; Siegel, Steven J. 2011. "Poly Lactic-co-Glycolic Acid (PLGA) as Biodegradable Controlled Drug Delivery Carrier." Polymers 3, no. 3: 1377-1397.

184.Makino, K. and Shibata, A. 2006, Chapter 2: Surface Properties of Liposomes Depending on Their Composition Volume 4, 49-77.

185.Manjunath K. and Reddy J.S., Venkateswarlu V., Solid lipid nanoparticles as drug delivery systems, Methods Find. Exp. Clin. Pharmacol. 27 (2005) 127144.

186.McArthur, J.C., Brew, B.J., Nath, A., (2005), Neurological complications of HIV infection. Lancet Neurol. 4 (9), 543-555.

187.McGee B., Smith N., Aweeka F. (2006), HIV pharmacology: barriers to the eradication of HIV from the CNS, HIV Clin. Trials 7: 142-153.

188.McNeil, S.E. 2005, Nanotechnology for the biologist J Leukoc Biol, 78, 585594.

189.Mehnert W. and Mader K., Solid lipid nanoparticles: production, characterization and applications, Adv. Drug Deliv. Rev. 47 (2001) 165-196

190.Menon LG, Kelly K, Yang HW, et al. Human bone marrow-derived mesenchymal stromal cells expressing S-TRAIL as a cellular delivery vehicle for human glioma therapy. Stem Cells. 2009 Sep;27(9):2320-30.

191.Messmer D., Hatsukari I., Hitosugi N., Schmidt-Wolf I. G. H., and Singhal P. C., "Morphine reciprocally regulates IL-10 and IL-12 production by monocytederived human dendritic cells and enhances $\mathrm{T}$ cell activation," Molecular Medicine, vol. 12, no. 11-12, pp. 284-290, 2006.

192.Meyer, O., Papahadjopoulos, D. and Leroux, J.C. (1998) Co- polymers of Nisopropylacrylamide can trigger $\mathrm{pH}$ sensitivity to stable liposomes. FEBS Lett. 421, 61-64.

193.Minn A, Ghersi-Egea JF, Perrin R, Leininger B and Siest G (1991) Drug metabolizing enzymes in the brain and cerebral microvessels. Brain Res Brain Res Rev 16:65-82. 
194.Mitra, S., Gaur, U., Ghosh, P.C. and Maitra, A.N. (2001) Tumour targeted delivery of encapsulated dextran doxorubicin conjugate using chitosan nanoparticles as carrier. J. Control. Release, 74, 317-323.

195.MMWR (2001), The Global HIVIAIDS epidemic, 2001. June 1; 50(21): 434456.

196.Mohanraj, V.J. and Chen, Y. 2006, Nanoparticles-A review Tropical Journal of Pharmaceutical Research, 5, 561-573.

197.Moorman J., Zhang Y., B. Liu, LeSage G., Chen Y., Stuart C., Prayther D, and Yin D. "HIV-1 gp120 primes lymphocytes for opioid-induced, beta-arrestin 2-dependent apoptosis," Biochimica et Biophysica Acta, vol. 1793, pp. 13661371, 2009.

198.Muller FJ, Snyder EY, Loring JF. Gene therapy: can neural stem cells deliver? Nat Rev Neurosci.2006 Jan;7(1):75-84.

199.Muller R.H., Ruhl D., Runge S., Schulze-Forster K., Mehnert. Cytotoxicity of solid lipid nanoparticles as a function of the lipid matrix and the surface, Pharm. Res. 14 (1997) 458-462

200.Muthana M, Scott SD, Farrow N, et al. A novel magnetic approach to enhance the efficacy of cell-based gene therapies. Gene Therapy. 2008;15:902-910.

201.Bertrand N., P. Simard, J.C. Leroux, Serum-stable, long-circulating, pHsensitive PEGylated liposomes, Methods Mol. Biol. 605 (2010) 545-558.

202.Nair, M.P., Pottathil, R., Heimer, E.P. and Schwartz, S.A. Immunoregulatory activities of human immunodeficiency virus (HIV) proteins: effect of HIV recombinant and synthetic peptides on immunoglobulin synthesis and proliferative responses by normal lymphocytes. Proc Natl Acad Sci U S A, 1988. 85(17): 6498-502.

203.Nair, MPN and Saiyed, Z (2011). Antiretroviral nanotherapies. In: The Neurology of AIDS, Third Edition. Eds: H.E. Gendelman, I.P. Everall, H.S. Fox, H.A. Gelbard, I. Grant, S.A. Lipton, S. Swindells. Oxford University Press, New York, 2011; pp 999-1004.

204.Nath A, Anderson C, Jones M, Maragos W, Booze R, Mactutus C, Bell J, Hauser KF, Mattson M. "Neurotoxicity and dysfunction of dopaminergic systems associated with AIDS dementia," Journal of Psychopharmacology, vol. 14, no. 3, pp. 222-227, 2000. 
205.Nath A, Hauser KF, Wojna V, Booze RM, Maragos W, Prendergast M, Cass W, Turchan JT. Molecular basis for interactions of HIV and drugs of abuse. J Acquir Immune Defic Syn.2002;31:S62-S69.

206.Nath A., Hauser K. F., Wojna V.,Booze R. M., Maragos W., Prendergast M., Cass W. and Turchan J.T "Molecular basis for interactions of HIV and drugs of abuse," Journal of Acquired Immune Deficiency Syndromes, vol. 31, supplement 2, pp. S62-S69, 2002.

207.Neff CP, Zhou J, Remling L, Kuruvilla J, Zhang J, Li H, Smith DD, Swiderski P, Rossi JJ, Akkina R. An aptamer-siRNA chimera suppresses HIV-1 viral loads and protects from helper CD4+ T cell decline in humanized mice. Sci Transl Med 3(66):66ra66, 2011.

208.Nestler EJ (2001) Molecular basis of long-term plasticity underlying addiction. Nat Rev Neurosci 2: 119-128.

209.Nijhuis M, van Maarseveen NM, Boucher CA. Antiviral resistance and impact on viral replication capacity: evolution of viruses under antiviral pressure occurs in three phases. Handb Exp Pharmacol.2009;189:299-320.

210.Nolan D, Reiss P, Mallal S. Adverse effects of antiretroviral therapy for HIV infection: a review of selected topics. Expert Opin Drug Saf. 2005;4:201-18.

211.Nowacek A, Gendelman HE. NanoART, neuroAIDS and CNS drug delivery. Nanomedicine (Lond)2009 Jul;4(5):557-74.

212.Nowacek AS, McMillan J, Miller R, et al. Nanoformulated Antiretroviral Drug Combinations Extend Drug Release and Antiretroviral Responses in HIV-1Infected Macrophages: Implications for NeuroAIDS Therapeutics.

213. Olivier J.C., Drug transport to brain with targeted nanoparticles, NeuroRx 2 (2005) 108-119.

214.Pan G., Giri N., Elmquist W.F. Abcg2/Bcrp1 mediates the polarized transport of antiretroviral nucleosides abacavir and zidovudine. Drug Metab. Dispos. 2007;35:1165-1173.

215.Pan, Y.; Du, X.; Zhao, F.; Xu, B., Magnetic Nanoparticles for the Manipulation of Proteins and Cells. Chem Soc Rev 2012, 41, 2912-2942.

216.Pardridge WM. Blood-brain barrier delivery. Drug Discovery Today. 2007;12:54-61.

217.Pardridge WM. Brain drug targeting: the future of brain drug development: Cambridge Univ Pr; 2001. 
218.Pardridge WM. Drug transport in brain via the cerebrospinal fluid. Fluids Barriers CNS 2011;8(1):7.

219.Park, J.H., Saravanakumar, G., Kim, K., and Kwon, I.C. 2010, Targeted delivery of low molecular drugs using chitosan and its derivatives Advanced Drug Delivery Reviews, 62, 28-41.

220.Pawlowski NA, Kaplan G, Abraham E, et al. The selective binding and transmigration of monocytes through the junctional complexes of human endothelium. J Exp Med. 1988 Nov 1;168(5):1865-82.

221.Pelkmans L, Kartenbeck J, and Helenius A (2001) Caveolar endocytosis of simian virus 40 reveals a new two-step vesicular-transport pathway to the ER. Nat Cell Biol 3: 473-483.

222.Peng, A., et al., PEGylation of a Factor VIII-Phosphatidylinositol Complex: Pharmacokinetics and Immunogenicity in Hemophilia A Mice. The AAPS Journal, 2012. 14(1): p. 35-42.

223.Peng, Z.G., K. Hidajat, and M.S. Uddin, Adsorption of bovine serum albumin on nanosized magnetic particles. Journal of Colloid and Interface Science, 2004. 271(2): p. 277-283.

224.Pereira M., Oliveira E. de, Garcion N.V., Benoit J.P., Couet W., Olivier J.C., Tissue distribution of indinavir administered as solid lipid nanocapsule formulation in mdr1a $\left(^{+/+}\right)$and mdr1a $\left({ }^{-/-}\right)$CF-1 mice, Pharm. Res. 22 (2005) 1898-1905.

225.Perelson AS, Essunger P, Cao Y. (1997), Decay characteristics of HIV-1 infected compartments during combination therapy. Nature, 387:188-191.

226.Persidsky Y., Heilman D., Haorah J., Zelivyanskaya M., Persidsky R., Weber G. A., Shimokawa H., Kaibuchi K. and Ikezu T. (2006) Rho-mediated regulation of tight junctions during monocyte migration across blood-brain barrier in HIV-1 encephalitis (HIVE). Blood 107, 4770-4780.

227.Peterson P. K., Gekker G., Hu S., Anderson Wr, Kravitz F, Porotoghese PS., Balfour H. H., Jr. and Chao C. C. "Morphine amplifies HIV-1 expression in chronically infected promonocytes cocultured with human brain cells," Journal of Neuroimmunology, vol. 50, no. 2, pp. 167-175, 1994.

228.Peterson P. K., Gekker G., Schut R., Hu S., Balfour H. H., Jr. and Chao C. C. (1993) Enhancement of HIV-1 replication by opiates and cocaine: The cytokine connection. Adv. Exp. Med. Biol. 335, 181-188.

229.Peterson P. K., Molitor T. W. and Chao C. C. (1998) The opioid-cytokine connection. J. Neuroimmunol. 83, 63-69. 
230.Peterson PK, Gekker G, Hu S, Lokensgard J, Portoghese PS, and Chao CC (1999) Endomorphin-1 potentiates HIV-1 expression in human brain cell cultures: implication of an atypical $\mu$-opioid receptor. Neuropharmacology 38: 273-278.

231.Pilakka-Kanthikeel S., Atluri, V.S.R, Sagar V., and Nair, M., Targeted brain derived neurotropic factors (BDNF) delivery across the blood-brain barrier for neuro-protection using magnetic nano carriers: an in-vitro study. PLOS ONE (2013), Volume 8 (4): e62241.

232.Pion, M., Serramia, M.J., Diaz, L., Bryszewska, M., Gallart, T., García, F., Gómez, R., de la Mata, F.J., Muñoz-Fernandez, M.Á. (2010). Phenotype and functional analysis of human monocytes-derived dendritic cells loaded with a carbosilane dendrimer. Biomaterials, Vol. 31, pp. 8749-8758.

233.Popescu MA, Toms SA. In vivo optical imaging using quantum dots for the management of brain tumors. Expert Rev Mol Diagn. 2006 Nov;6(6):879-90.

234.Prior S., Gander B., Blarer N., Merkle H.P., Subira M.L., Irache J.M., Gamazo C., In vitro phagocytosis and monocyte-macrophage activation with poly(lactide) and poly(lactide-co-glycolide) microspheres, Eur. J. Pharm. Sci. 15 (2002) 197-207.

235. Qiao, R., et al., Receptor-Mediated Delivery of Magnetic Nanoparticles across the Blood-Brain Barrier. ACS Nano, 2012. 6(4): p. 3304-3310.

236.R. Mallipeddi, L.C. Rohan, Progress in antiretroviral drug delivery using nanotechnology, Int. J. Nanomedicine 5 (2010) 533-547.

237.Rao KS, Reddy MK, Horning JL, et al. TAT-conjugated nanoparticles for the CNS delivery of anti-HIV drugs. Biomaterials. 2008;29:4429-38.

238.Reddy LH, Vivek K, Bakshi N, Murthy RSR. Tamoxifen citrate loaded solid lipid nanoparticles $\left(S L N^{\top M}\right)$ : preparation, characterization, in vitro drug release, and pharmacokinetic evaluation.Pharmaceutical Development and Technology. 2006;11(2):167-177.

239.Reddy M.K. and Labhasetwar V., Nanoparticle-mediated delivery of superoxide dismutase to the brain: an effective strategy to reduce ischemiareperfusion injury, FASEB J. 23 (2009) 1384-1395.

240.Reddy PVB, Pilakka-Kanthikeel S, Saxena SK, Saiyed Z, Nair, MP. (2012), Interactive Effects of Morphine on HIV Infection: Role in HIV-Associated Neurocognitive Disorder. AIDS Research and Treatment, 2012:Article ID 953678. 
241.Rempe R., Cramer S., Huwel S., Galla H.J., Transport of Poly(n-butylcyanoacrylate)nanoparticles across the blood-brain barrier in vitro and their influence on barrier integrity, Biochem. Biophys. Res. Commun. 406 (2011) 64-69.

242.Rempe, R., et al., Transport of Poly(n-butylcyano-acrylate) nanoparticles across the blood-brain barrier in vitro and their influence on barrier integrity. Biochemical and Biophysical Research Communications, 2011. 406(1): p. 6469.

243.Resnick L., Berger J.R., Shapshak P., Tourtellotte W.W. (1988), Early penetration of the blood brain barrier by HIV. Neurology.;38:9-14.

244.Resnick, L., diMarzo-Veronese, F., Schubbach, J., Tourtellotte, W. W., Ho, D. D., Muller, F., Shapshak, P., Vogt, M., Groopman, J. E., Markham, P. D. and Gallo, R. C. (1985), Intra blood brain barrier synthesis of HTLV-111 specific IgG in patients with neurologic symptoms associated with AIDS or AIDS-related complex. New England Journal of Medicine, 313: 1498-1504.

245.Reynolds JL, Law WC, Mahajan SD, Aalinkeel R, Nair B, Sykes DE, Mammen MJ, Yong KT, Hui R, Prasad PN, Schwartz SA. Morphine and galectin-1 modulate HIV-1 infection of human monocyte-derived macrophages. J Immunol. 2012;8:3757-3765.

246. Reynolds JL, Law WC, Mahajan SD, Aalinkeel R, Nair B, Sykes DE, Yong KT, Hui R, Prasad PN, Schwartz SA. Nanoparticle Based Galectin-1 Gene Silencing, Implications in Methamphetamine Regulation of HIV-1 Infection in Monocyte Derived Macrophages. J Neuroimmune pharmacol. Sep 2012, $7(3): 673-85$.

247.Riddle EL, Fleckenstein AE, Hanson GR. Mechanisms of Methamphetamine-induced Dopaminergic Neurotoxicity. (2006), AAPS Journal. 8(2): E413-E418.

248.Rivera-Amill V., Silverstein P. S., Noel R. J., Kumar S., and Kumar A., "Morphine and rapid disease progression in nonhuman primate model of AIDS: inverse correlation between disease progression and virus evolution," Journal of Neuroimmune Pharmacology, vol. 5, no. 1, pp. 122132, 2010.

249.Riviere C, Martina MS, Riviere C, et al. Magneting targeting of nanometric magnetic fluid loaded liposomes to specific brain intravascular areas: A dynamic imaging study in mice. Radiology.2007;244:439-448.

250.Robinson TE, Kolb B (1999) Alterations in the morphology of dendrites and dendritic spines in the nucleus accumbens and prefrontal cortex following 
repeated treatment with amphetamine or cocaine. Eur J Neurosci 11: 15981604.

251.Rogers T. J., Steele A. D., Howard O. M. and Oppenheim J. J. (2000) Bidirectional heterologous desensitization of opioid and chemokine receptors. Ann. N. Y. Acad. Sci. 917, 19-28.

252.Rogers TJ. Immunology as it pertains to drugs of abuse, AIDS and the neuroimmune axis: mediators and traffic. J Neuroimmune Pharmacol 2011;6:20-27.

253.Rojavin M., Szabo I., Bussiere J. L., Rogers T. J., Adler M. W., and Eisenstein T. K., "Morphine treatment in vitro or in vivo decreases phagocytic functions of murine macrophages," Life Sciences, vol. 53, no. 12, pp. 9971006, 1993.

254.Rolfs A and Schuhmacher HC (1990), Early findings in the cerebrospinal fluid of patients with HIV-1 infection of the central nervous system. N Engl J Med 323:418-419.

255.Ronaldson P.T., Persidsky Y., Bendayan R., Regulation of ABC membrane transporters in glial cells: relevance to the pharmacotherapy of brain HIV-1 Infection,

256.Roy, U, Balkundi S., McMillan, J., Gendelman, H.E. Nanoformulated Medicines. In: The Neurology of AIDS, Third Edition. Eds: H.E. Gendelman, I.P. Everall, H.S. Fox, H.A. Gelbard, I. Grant, S.A. Lipton, S. Swindells. Oxford University Press, New York, 2011; pp 987-998.

257.Rumbaugh JA, Steiner J, Sacktor N, Nath A. Developing neuroprotective strategies for treatment of HIV-associated neurocognitive dysfunction. Futur HIV Ther 2008; 2: 271-280.

258.S. Hu, W. S. Sheng, J. R. Lokensgard, and P. K. Peterson, "Morphine potentiates HIV-1 gp120-induced neuronal apoptosis," Journal of Infectious Diseases, vol. 191, no. 6, pp. 886-889, 2005.

259.Saiyed ZM, Parasramka M, Telang SD, Ramchand CN (2007) Extraction of DNA from agarose gel using magnetic nanoparticles (magnetite or $\mathrm{Fe} 3 \mathrm{O} 4$ ). Anal Biochem 363: 288-290.

260.Sarti F, Borgland SL, Kharazia VN, Bonci A (2007) Acute cocaine exposure alters spine density and long-term potentiation in the ventral tegmental area. Eur J Neurosci 26: 749-756. 
261.Saurer T. B., Carrigan K. A., ljames S. G., and Lysle D. T., "Suppression of natural killer cell activity by morphine is mediated by the nucleus accumbens shell," Journal of Neuroimmunology, vol. 173, no. 1-2, pp. 3-11, 2006.

262.Semete B, Kalombo L, Katata L and Swai H (2010), Nano-drug delivery systems: Advances in TB, HIV and Malaria treatment, Smart Biomol. Medicine, Edited by Ajay K. Mishra, Ashutosh Tiwari, and Shivani B. Mishra, Smar Biomol Medicine, VBSRI press, PP 15-52.

263.Senyei A, Widder K, Czerlinski C. Magnetic guidance of drug carrying microspheres. J Appl Phys. 1978;49:3578-83.

264.Sershen, S.R., Westcoot, S.L., Halas, N.J. and West, J.L. (2000) Temperature-sensitive polymer-nanoshell com-posites for photothermally modulated drug delivery. J. Biomed. Mater. Res., 51, 293-298.

265.Shaik N, Pan G, Elmquist WF. Interactions of pluronic block copolymers on P-gp efflux activity: experience with HIV-1 protease inhibitors. J Pharm Sci. 2008;97(12):5421-5433.

266.Shaik N., Giri N., Pan G., Elmquist W.F. P-glycoprotein-mediated active efflux of the anti-HIV1 nucleoside abacavir limits cellular accumulation and brain distribution. Drug Metab. Dispos.2007;35:2076-2085.

267.Sharma A., Sharma U.S. (1997). Liposomes in drug delivery: progress and limitations. International Journal Pharmaceutics, Vol. 154, pp. 123-140.

268.Sharma P., Garg S. (2010). Pure drug and polymer based nanotechnologies for the improved solubility, stability, bioavailability and targeting of anti-HIV drugs. Advanced Drug Delivery Reviews, Vol. 62, pp. 491-502.

269. Shively L, Chang L, LeBon JM, Liu Q, Riggs AD, Singer-Sam J(2003) Realtime PCR assay for quantitative mismatch detection. Biotechniques 34:498502.

270.Siegel GJ, Agranoff BW, Albers RW, et al., editors. Basic Neurochemistry: Molecular, Cellular and Medical Aspects. 6th edition. Philadelphia: LippincottRaven; 1999. Blood-Cerebrospinal Fluid Barrier. Available from: http://www.ncbi.nlm.nih.gov/books/NBK27998/

271.Simonato M. The neurochemistry of morphine addiction in the neocortex. Trends Pharmacol.Sci. 1996; 17, 410-415.

272.Singer EJ, Valdes-Sueiras M, Commins D, Levine A (2010), Neurologic presentations of AIDS. Neurol Clin 28: 253-275. 
273.Singhal PC, Kapasi AA, Reddy K, Franki N, Gibbons N, et al. (1999) Morphine promotes apoptosis in Jurkat cells. J Leukoc Biol 66: 650-658.

274.Singhal PC, Sharma P, Kapasi AA, Reddy K, Franki N, et al. (1998) Morphine enhances macrophage apoptosis. J Immunol 160: 1886-1893.

275.Skar H, Hedskog C, Albert J. (2011) HIV-1 evolution in relation to molecular epidemiology and antiretroviral resistance. Annals of the New York Academy of Sciences.1230:108-18.

276.Smith DL, Pozueta J, Gong B, Arancio O, Shelanski M (2009) Reversal of long-term dendritic spine alterations in Alzheimer disease models. Proc Natl Acad Sci U S A 106: 16877-16882.

277.Smith GH (2005) Opening statement of Senator Gordon H. Smith. Aging hearing: HIV over fifty, exploring the new threat. Senate Committee on Aging, Washington, DC, U.S. Government Printing Office.

278.Sollner T, Bennett MK, Whiteheart SW, et al. A protein assemblydisassembly pathway in vitro that may correspond to sequential steps of synaptic vesicle docking, activation, and fusion. Cell. 1993 Nov 5;75(3):40918.

279.Soppimath, K.S., Aminabhavi, T.M., Kulkarni, A.R., and Rudzinski, W.E. 2001, Biodegradable polymeric nanoparticles as drug delivery devices Journal of Controlled Release, 70, 1-20.

280.Spitzenberger T.J., Heilman D., Diekmann C., Batrakova E.V., Kabanov A.V., Gendelman H.E., Elmquist W.F., Persidsky Y., Novel delivery system enhances efficacy of antiretroviral therapy in animal model for HIV-1 encephalitis, J. Cereb. Blood Flow Metab. 27 (2007) 1033-1042.

281.Sporer B, Paul R, Koedel U, Grimm R, Wick M, Goebel FD, Pfister HW. Presence of matrix metalloproteinase- 9 activity in the cerebrospinal fluid of human immunodeficiency virus-infected patients. J Infect Dis. 1998;178:854857.

282.Studeny M, Marini FC, Champlin RE, Zompetta C, Fidler IJ, Andreeff M. Bone marrow-derived mesenchymal stem cells as vehicles for interferon-beta delivery into tumors. Cancer Res. 2002 Jul 1;62(13):3603-8.

283.Sun Y-k., Ma M., Zhang, Y. and Gu, N. (2004). Synthesis of nanometer-size maghemite particles from magnetite. Colloids and Surfaces A: Physicochemical and Engineering Aspects, 245(1-3): p. 15-19. 
284.Sun, J., Li, Y., Liang, X-J., Wang, P.C. (2011). Bacterial Magnetosome: A Novel Biogenetic Magnetic Targeted Drug Carrier with Potential Multifunctions. Journal of Nanomaterials, 2011: Article ID 469031

285.Suri SS, Fenniri H, Singh B (2007) Nanotechnology-based drug delivery systems. J Occup Med Toxicol 2: 16.

286.Svenson S., Dendrimers as versatile platform in drug delivery applications, Eur. J. Pharm. Biopharm. 71 (2009) 445-462.

287.Tamai I and Tsuji A (2000) Transporter-mediated permeation of drugs across the blood-brain barrier. J Pharm Sci 89:1371-1388.

288.Taubenfeld SM, Wiig KA, Bear MF, Alberini CM (1999) A molecular correlate of memory and amnesia in the hippocampus. Nat Neurosci 2: 309-310.

289.TheWorldBank(2012), http://www.unaids.org/en/aboutunaids/unaidscosponsors/theworldbank/.

290.Thiele L, Merkle HP, Walter E. Phagocytosis and phagosomal fate of surface-modified microparticles in dendritic cells and macrophages. Pharm Res. 2003 Feb;20(2):221-8.

291.Tombacz, E.; Majzik, A.; Horvat, Z. S.; Illes, E. (2006) Magnetite in aqueous medium: Coating its surface and surface coated with it. Romanian Reports in Physics, Vol. 58, No. 3, P. 281-286.

292.Torchilin VP. Recent advances with liposomes as pharmaceutical carriers. Nat Rev Drug Discov.2005;4:145-160.

293.Trivedi R, Kompella UB. Nanomicellar formulations for sustained drug delivery: strategies and underlying principles. Nanomedicine. 2010;5:485505.

294.Tsuji A and Tamai II (1999) Carrier-mediated or specialized transport of drugs across the blood-brain barrier. Adv Drug Deliv Rev 36:277-290.

295.UNAIDS (2010), Global Report: UNAIDS report on the global AIDS epidemic 2010.

296.UNAIDS (2012 a), Fact Sheet: Women, girls, gender equality and HIV.

297.UNAIDS (2012b), Fact Sheet: Adolescents, young people and HIV.

298.UNAIDS $\left(2012_{\mathrm{c}}\right)$, World AIDS day report 2011. 
299.UNAIDS/PCB (2009), HIV prevention among injecting drug users. In, 24th Meeting of the UNAIDS Programme Coordinating Board, Geneva, Switzerland 22-24 June 2009, UNAIDS/PCB(24)/09.9.Rev.1.

300.UNESCO(2012),

http://www.unaids.org/en/aboutunaids/unaidscosponsors/unesco/.

301.UNODC(2012),

http://www.unaids.org/en/aboutunaids/unaidscosponsors/unodc/.

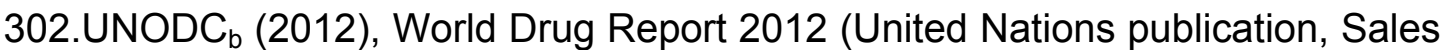
No. E.12.XI.1).

303.Varatharajan $L$ and Thomas SA. (2009), The transport of anti-HIV drugs across blood-CNS interfaces: summary of current knowledge and recommendations for further research. Antiviral Res; 82(2):A99-A109.

304.Vella S, Palmisano L. The global status of resistance to antiretroviral drugs. Clin Infect Dis.2005;41(Suppl 4):S239-46.

305.Vert M, Domurado D. Poly(ethylene glycol): protein-repulsive or albumincompatible? J Biomater Sci Polym Ed. 2000;11:1307-17.

306.Viola H, Furman M, Izquierdo LA, Alonso M, Barros DM, et al. (2000) Phosphorylated CAMP response element-binding protein as a molecular marker of memory processing in rat hippocampus: effect of novelty. J Neurosci 20: RC112.

307.Vitas et al., (1996) Effect of composition and method of preparation of liposomes on their stability and interaction with murine monocytes infected with Brucella abortus. Antimicrobial Agents and Chemotherapy 40(1) 146151.

308. Vivithanaporn P, Gill MJ, Power C (2011). Impact of current antiretroviral therapies on neuroAIDS. Expert Rev. Anti-Infect. Ther. 9:371-374.

309.Vyas T. K., Shahiwala A., Amiji M. M. (2008). Improved oral bioavailability and brain transport of saquinavir upon administration in novel nanoemulsion formulations. International Journal of Pharmaceutics, Vol. 347, pp. 93-101.

310.Vyas TK, Shah L, Amiji MM. Nanoparticulate drug carriers for delivery of HIVIAIDS therapy to viral reservoir sites. Expert Opin Drug Deliv. 2006;3(5):613-628.

311.Wang DD, Bordey A. The astrocyte odyssey. Prog Neurobiol. 2008;86(4):342-67. 
312.Wang J., Barke R. A., Ma J., Charboneau R., and Roy S., "Opiate abuse, innate immunity, and bacterial infectious diseases," Archivum Immunologiae et Therapia Experimentalis (Warsz), vol. 56, pp. 299-309, 2008.

313.Weiss J., Theile D., Ketabi-Kiyanvash N., Lindenmaier H., Haefeli W.E. Inhibition of MRP1/ABCC1, MRP2/ABCC2, and MRP3/ABCC3 by nucleoside, nucleotide, and non-nucleoside reverse transcriptase inhibitors. Drug Metab. Dispos. 2007;35:340-344.

314.Weksler B, Subileau E, Perriere N, Charneau P, Holloway K, et al. (2005) Blood-brain barrier-specific properties of a human adult brain endothelial cell line. The FASEB journal 19: 1872-1874.

315.WHO (2012), http://www.unaids.org/en/aboutunaids/unaidscosponsors/who/.

316.Wilson JK, Sargent JM, Elgie AW, Hill JG, Taylor CG. A feasibility study of the MTT assay for chemosensitivity testing in ovarian malignancy. British Journal of Cancer. 1990;62(2):189-194.

317.Wiogo H.T. R., Lim M., Bulmus V., Gutierrez L., Woodward R. C., and Amal R. (2012), Insight into Serum Protein Interactions with Functionalized Magnetic Nanoparticles in Biological Media. Langmuir, 2012, 28 (9), pp 4346-4356.

318.Wolka AM, Huber JD and Davis TP (2003) Pain and the blood-brain barrier: obstacles to drug delivery. Adv Drug Deliv Rev 55:987-1006.

319.Wong H.L., Rauth A.M., Bendayan R., Wu X.Y., Combinational treatment with doxorubicin and GG918 (Elacridar) using polymer-lipid hybrid nanoparticles (PLN) and evaluation of strategies for multidrug-resistance reversal in human breast cancer cells, J. Control. Release 116 (2006) 275284.

320.Wong HL, Chattopadhyay N, Wu XY, Bendayan R. Nanotechnology applications for improved delivery of antiretroviral drugs to the brain. Advanced Drug Delivery Reviews. 2010;62(4-5):503-517.

321. Wong HL, Wu XY and Bendayan R. 2012. Nanotechnological advances for the delivery of CNS therapeutics. Adv Drug Deliv Rev, 64 (2012) 686-700.

322.Woods SP, Moore DJ, Weber E, Grant I ( 2009): Cognitive neuropsychology of HIV-associated neurocognitive disorders.Neuropsychol Rev 19: 152-168.

323.Wu, X., Yuan, G., Brett, C. M., Hui, A. C. and Giacomini, K. M.: Sodium dependent nucleoside transport in choroid plexus from rabbit. J. Biol. Chem. 267: 8813-8818, 1992 
324.Xia Y, Wang CZ, Liu J, Anastasio NC, Johnson KM (2010) Brain-derived neurotrophic factor prevents phencyclidine-induced apoptosis in developing brain by parallel activation of both the ERK and PI-3K/Akt pathways. Neuropharmacology 58: 330-336.

325.Xu, G., et al., Bioconjugated quantum rods as targeted probes for efficient transmigration across an in vitro blood-brain barrier. Bioconjugate Chemistry, 2008. 19(6): p. 1179-1185.

326.Yin D, Mufson RA, Wang R, Shi Y (1999) Fas-mediated cell death promoted by opioids. Nature 397: 218.

327.Yong Liu Y, , Niu T-S, Zhang L, Yang J-S (2010), Review on nano-drugs. 2(1): 41-48 .

328.Yoo J-W (2012), Toward Improved Selectivity of Targeted Delivery: The Potential of Magnetic Nanoparticles. Arch Pharm Res Vol 35, No 1, 1-2, 2012.

329.Yoo JY, Kim JM, Seo KS, et al. Characterization of degradation behavior for PLGA in various $\mathrm{pH}$ condition by simple liquid chromatography method. Biomed Mater Eng. 2005;15:279-88.

330.Yoo, H.S., Lee, E.A. and Park, T.G. (2002) Doxorubicin- conjugated biodegradable polymeric micelles having acid-cleavable linkages. J. Control. Release, 82, 17-27.

331.Yousif S, Marie-Claire C, Roux F, Scherrmann JM, Declèves X. (2007) Expression of drug transporters at the blood-brain barrier using an optimized isolated rat brain microvessel strategy. Brain Res 1134:1-11.

332.Yu, S., et al., Magnetic and $\mathrm{pH}$-sensitive nanoparticles for antitumor drug delivery. Colloids and Surfaces B: Biointerfaces, 2013. 103(0): p. 15-22.

333.Zhang N. and Oppenheim J. J., "Crosstalk between chemokines and neuronal receptors bridges immune and nervous systems," Journal of Leukocyte Biology, vol. 78, no. 6, pp. 1210-1214, 2005.

334.Zhang X., Xie J., Li S., Wang X., Hou X., The study on brain targeting of the amphotericin B liposomes, J. Drug Target. 11 (2003) 117-122.

335.Zhang Z, Feng SS. The drug encapsulation efficiency, in vitro drug release, cellular uptake and cytotoxicity of paclitaxel-loaded poly(lactide)-tocopheryl polyethylene glycol succinate nanoparticles.Biomaterials. 2006;27:4025-33. 
336.Zhang, Zhiqi, "Blood-Brain Barrier in vitro Model: A Tissue Engineering Approach and Validation" (2010). FIU Electronic Theses and Dissertations. Paper 246. http://digitalcommons.fiu.edu/etd/246.

337.Zhao Y, Haney MJ, Klyachko NL, et al. Polyelectrolyte complex optimization for macrophage delivery of redox enzyme nanoparticles. Nanomedicine (Lond) 2011 Jan;6(1):25-42. 
VITA

VIDYA SAGAR

EDUCATION:

2001-2005

Bachelor of Fisheries Science

College of Fisheries, Orissa University of Agriculture \&

Technology, Berhampur, Orissa, India

2005-2007

Masters in Fisheries Science (Nutrition \& Biochemistry), Central Institute of Fisheries Education, Mumbai, India

2011-2012

Doctoral Candidate in Biology

Florida International University, Miami, FI, USA

2005

Diploma in Computer Science

National Institute of Advanced Technology, Orissa, India

PROFESSIONAL EXPERIENCE:

2006-2007

Training program faculty

Central Institute of Fisheries Education, Kakinada Center, Andhra Pradesh, India

2007-2008

Research fellow

Central Institute of Fisheries Education, Mumbai, India

2008-2013

Teaching assistant

Florida International University, Miami, FI, USA

GRANTS:

- Student Summer Research Award 2011, MBRS-RISE Biomedical Research Initiative, Florida International University, Miami

Project: "Sequestration of eukaryotic translation apparatus: Mechanism(s) utilized by Mammalian Reovirus (MRV) to shut off host translation".

- Student Summer Research Award 2013, MBRS-RISE Biomedical Research Initiative, Florida International University, Miami

Project: "Magnetic nanoparticle-based targeted drug delivery for treatment of neuro-AIDS and drug addiction". 


\section{PRESENTATIONS:}

- Vidya Sagar, Pilakka-Kanthikeel S. K., Priestap, H., Atluri, V.S.R, Ding, H., Khizroev, S., and Nair, M.P. Magnetic-nanoformulation of $\mu$-opioid receptor antagonist (CTOP) for treatment of morphine-induced neuropathogenesis during HIV infection. Society of Neurolmmune Pharmacology- $19^{\text {th }}$ Scientific Conference, San Juan, Puerto Rico, April 3-6 ${ }^{\text {th }}$, 2013. Journal of Neurolmmune Pharmacology, 8(2): 432-433. [Poster]

- Hong Ding, Marisela Agudelo, Sudheesh Pilakka-Kanthikeel, Rakesh Guduru, Vidya Sagar, Venkata Atluri, Samikkannu Thangavel, and Madhavan Nair. Dural mechanism enhanced blood-brain-barrier crossing by transferrin conjugated fluorescent magnetic liposomes. Society of Neurolmmune Pharmacology-19 ${ }^{\text {th }}$ Scientific Conference, San Juan, Puerto Rico, April 3-6 $6^{\text {th }}$, 2013. Journal of Neurolmmune Pharmacology, 8(2): 414. [Poster]

- Raymond A. D., Agudelo, M., Yndart, A., Alturi, V., Pilakka, S., Pichili, V., Hong, D., Sagar, V., Thangavel, S., Rao, K.V.K., Powell, M.D., and Nair, M. Morphine and exosomal HIV-1 Nef: Potential roles in NeuroAIDS development. Health Disparity Conference, July $11^{\text {th }}-13^{\text {th }}, 2012$, University of North Texas, Texas. [Poster]

- Vidya Sagar and Kenneth E. Murray. The mammalian orthoreovirus bicistronic M3 mRNA initiates translation using a cap-independent scanning mechanism that does not require mRNA circularization. American Society for Virology- $30^{\text {th }}$ Annual meeting-2011, Minneapolis, Minnesota, July $16-20^{\text {th }}$, Scientific program and abstracts; W28-3, pp 159. [Oral presentation]

- Vidya Sagar and Kenneth E. Murray. The untranslated regions of the mammalian orthoreovirus (MRV) M3 transcript do not possess cis-acting sequences or structures necessary for translation. American Society for Microbiology-Florida Branch Annual meeting-2010, Islamorada, Florida, October $9-10^{\text {th }}, 2010$. [Oral presentation]

\section{PEER-REVIEWED RESEARCH ARTICLES}

- M. Nair, R. Guduru, P. Liang, J. Hong, V. Sagar, and S. Khizroev, Externallycontrolled on-demand release of anti-HIV drug AZTTP using magneto-electric nanoparticles as carriers. Nature Communications (2013), 4: 1707.

- Pilakka-Kanthikeel S. K., Atluri, V.S.R, Sagar V., and Nair, M., Targeted brain derived neurotropic factors (BDNF) delivery across the blood-brain barrier for neuro-protection using magnetic nano carriers: an in-vitro study. PLOS ONE (2013), Volume 8 (4): e62241.

- Kumar Vikas, Sahu Narottam P., Pal Asim K., Kumar Shivendra, Sagar Vidya, Sinha Amit Kumar, Ranjan Jayant., 2010. Nucleic acid content changes of a tropical freshwater fish Labeo rohita fed gelatinized and nongelatinized starch diet. Journal of the World Aquaculture Society, 42(S2): 270-277.

- Vidya Sagar, N. P. Sahu, A. K. Pal., K. K. Jain, G. Venugopal. 2009. Growth and digestive enzymes of Macrobrachium rosenbergii juveniles: Effect of different stock type and dietary protein level under the similar culture environment. Aquaculture Research, 40(12): 1383-1393. 